\title{
Kewords
}

broadly neutralizing antibodies (bnAbs); knock-in (KI) mice; B-cell tolerance controls; B-cell anergy; receptor editing; HIV-1 mimicry; B-cell lineages; CD4-binding site (CD4bs); gp41 MPER; sequential immunization; unmutated common ancestor (UCA) 


\section{Overview of the current state of HIV vaccine research}

\subsection{Candidate protective HIV-1 responses}

After more than 30 years of HIV vaccine research, the correlates of immune protection against HIV-1 remain ill defined. In terms of a protective role for T cells, perhaps the strongest data supporting a critical for this component during vaccination comes from a recent immunization study in non-human primates, using attenuated Simian Immunodeficiency Virus (SIV)-expressing rhesus cytomegalovirus (rhCMV) vectors (Hansen et al., 2013a). In this study, rhCMV-vaccination cleared infection in approximately half of SIV-challenged macaques. Intriguingly, follow-up analyses established that the SIVexpressing rhCMV vector mediated atypical killing of SIV-infected CD4 T-cells, i.e. by CD8 T-cells that recognized either MHC class II or HLA E-restricted HIV antigens (Hansen et al., 2013b). The variability in protection from challenge (and the associated viral control) afforded by this regimen was surprising, given the protective pattern normally observed for CD8 T cell-mediated antiviral immunity is all-or-none, thus pointing to a strong host factorinfluenced (immunogenetic or other) component for this effect. Nevertheless, the most interesting potential correlate emerging from these key studies is that while a-HIV CD8 CTL activity fails to control transmitted/founder (T/F) virus escape during acute HIV infection (McMichael et al., 2010), it can do so in the setting of SIV-rhCMV vaccination.

The largest major obstacle facing HIV-1 vaccine research however, continues to be on the humoral side, namely, developing a vaccine approach capable of inducing a rapid and durable protective $\mathrm{Ab}$ response. While it is not known what spectrum of elicited functional antibodies could provide such protection, a key breakthrough for the field, right after two large phase III trials had failed, came when the RV144 trial results were reported in 2009. In this trial conducted in Thailand, the first statistically significant, albeit modest, reduction in HIV-1 acquisition were observed using a regimen consisting of the combination of a replication-defective canarypox vector (ALVAC) prime and a recombinant gp120 protein (AIDSVAX) boost (Rerks-Ngarm et al., 2009). While vaccine efficacy approached 60\% over the $1^{\text {st }}$ year, it over time and overall efficacy over the entire (3.5 year) trial period was $31 \%$. An immune correlates follow-up study identified V2-specific IgG Abs that associated with decreased transmission risk (Haynes et al., 2012a). While the RV144 vaccine surprisingly induced no neutralization of HIV primary isolates, the vaccine regimen did induce V2 antibodies that bound HIV-infected CD4 T-cells and mediated Antibody dependent cellular cytoxicity (ADCC). Thus, one potential correlate could be ADCC-mediated decrease in HIV acquisition (Haynes et al., 2012a; Tomaras et al., 2013).

As opposed to these seemingly more routinely-induced, candidate protective Ab responses elicited by the RV144 trial, it is widely held (for reasons that will be discussed directly below) that the "holy grail" of HIV vaccinology, namely, induction of broadly neutralizing antibodies (bnAbs), is critical to achieve, as part of an HIV vaccine regimen, for it to be truly preventative, and thus efficacious. However, to date, no immunization strategy can elicit such "difficult-to-induce" or "exceptional" Abs. A primary focus of this review is to discuss how humanized bnAb KI models, can enable more in-depth analysis of the challenges limiting the normally rare, but apparently desirable $\mathrm{Ab}$ traits immunization either have to be 
capable of selecting for, or generate, in order to confer bnAb function. More generally, this review will also cover how such KI models will a key technical gap in the filed: having available more iterative, robust, and practical vaccine testing platforms.

\subsection{The case for bnAbs as key components of an efficacious HIV vaccine}

Neutralizing antibodies (nAbs) are critical contributors to protective responses against most viral infections (Excler et al., 2014; Plotkin, 2010). However, several features of HIV-1, namely the unusually dense glycosylation of its envelope (Env), its rapid integration into host cells, and its high mutation rate, collectively distinguish it from all other viruses for which successful nAb-eliciting vaccines have been made (Mascola and Haynes, 2013). Each of these features poses a unique, unprecedented challenge for developing an efficacious vaccine for HIV-1. The exceptional degree of glycosylation shields most relevant Env epitopes, rendering it highly refractory to recognition by conventional Abs, and thus allowing efficient escape (Wei et al., 2003). The rapid establishment of a latently infected $\mathrm{CD}^{+}{ }^{+} \mathrm{T}$-cell pool necessitates viral transmission be completely blocked. At the population level, mutability of Env requires a humoral response to be adept in dealing with extreme viral diversity. Given these stringent requirements, a truly protective HIV-1 vaccine will most likely need to induce a rapid recall response involving the aforementioned broadly neutralizing antibodies (bnAbs), capable of neutralizing a wide spectrum of native HIV-1 strains, i.e. bearing highly-occluded (heavily-glycosylated) epitopes. Studies demonstrating absolute protection afforded by passive transfer (Hessell et al., 2009a; Hessell et al., 2009b) or transduction (Balazs et al., 2012) of monoclonal bnAbs, prior to viral challenge, support this notion. Added rationale for pursuing a bnAb-based HIV vaccine approach has recently been provided by demonstration that a single injection of bnAbs protects from repeated weekly challenge, for up to 6 months (Gautam et al., 2016).

\subsection{Characteristics of bnAbs isolated during infection and their Env targets: clues for vaccine design}

Although an HIV-1 vaccine regimen has yet to be designed that is capable of inducing detectable plasma bnAb responses (Haynes, 2015), relatively recent findings that bnAbs do develop over years, in a subset of HIV-infected subjects (Gray et al., 2011; Hraber et al., 2014; Mikell et al., 2011; Simek et al., 2009; Tomaras et al., 2011) has provided renewed momentum for the HIV-1 vaccine field to devise new immunization formulations and strategies to induce them. Improved memory B-cell sorting/culturing techniques and highthroughput Ig cloning methodologies, combined with increased numbers of large cohorts of HIV-1-infected subjects from which longitudinal samples are available, have enabled the discovery and characterization of many new monoclonal bnAbs over the past few years, providing two key insights for HIV vaccine design. First, the discovery and elegant characterization of these new HIV-1 bnAbs has provided exquisitely detailed structural information regarding five distinct, but conserved, regions in the Env trimer in which their epitopes cluster (Burton and Hangartner, 2016; Burton and Mascola, 2015), thus providing a blueprint of the vulnerable regions a bnAb-eliciting vaccine can exploit. One of these five bnAb targets is in the gp41 subunit, the membrane proximal external region (MPER), while the other four are in the gp120 subunit: the co-receptor CD4-binding site (CD4bs) and three 
peptide/glycan epitope-rich regions, either centered around the N160 glycosylation site in the stem of the V1/V2 loop (i.e. the trimer apex) or around the N332 glycosylation site in the V3 variable loop (i.e the high-mannose patch), or most recently, in the gp120/gp41 bridging interface.

The second major insight for vaccine design is that bnAbs, despite their independent origins, share an intriguing set of traits, which are atypical of conventional Abs. Specifically, in addition to utilizing a restricted set of human $\mathrm{V}_{\mathrm{H}}$ genes (Mascola and Haynes, 2013), all bnAbs isolated to date exhibit one or more of the following three unusual characteristics (Table 1): i) poly-/autoreactivity, ii) exceptionally long heavy chain complementaritydetermining regions i.e. HCDR3s, the critical antigen contact regions encoded by the V(D)J junctions of immunoglobulins (Ig), and iii) high levels of $\mathrm{V}(\mathrm{D}) \mathrm{J}$ somatic hypermutation (SHM), the latter also frequently accompanied by otherwise extremely rare insertion or deletion events (indels), whose origins are poorly understood but thought to be associated with elevated SHM. Indeed, it is the atypical nature of these hallmark features that likely equips bnAbs with their protective functions, i.e. by allowing them to overcome particular structural barriers in Env. For instance, elongated HCDR3s likely play a role in countering glycan occlusion of bnAb epitopes by penetrating the glycan shield (Pejchal et al., 2010), polyreactivity may allow B-cell receptor (BCR) "heteroligation" to deal with unusually low surface density of Env trimers on HIV virions (Mouquet et al., 2010) or to better access lipid-occluded epitopes (Alam et al., 2009), and extensive SHM may provide structural flexibility to counter natural variation in otherwise relatively-conserved bnAb-targeting regions (Burton and Hangartner, 2016; Klein et al., 2013; Kwong and Mascola, 2012; Mascola and Haynes, 2013; West et al., 2014).

It has now becoming increasingly clear that these same unusual bnAb traits also pose daunting immunologic obstacles to initiating and/or completing the maturation of bnAbs by vaccine strategies (reviewed in (Haynes et al., 2014; Verkoczy and Diaz, 2014; Verkoczy et al., 2011b)). For example, long HCDR3s and in vitro polyreactivity are negatively correlated with function, specificity, and/or frequency of B-cells in the pre-immune repertoire, due to difficulties in their Immunogloblulin (Ig) heavy chains (HCs) pairing to either surrogate or bona fide Ig light chains (LCs) and/or invoking central B-cell tolerance controls (Ivanov et al., 2005; Keenan et al., 2008; Meffre et al., 2001; Shiokawa et al., 1999; Wardemann et al., 2003). Furthermore, excess SHM generated during bnAb development during infection, if also required in the setting of vaccination, will invoke highly disfavored, protracted affinity maturation (AM) pathways (Batista and Neuberger, 1998; Haynes, Verkoczy and Kelsoe, 2014; Kepler and Perelson, 1993; Sabouri et al., 2014a) that seem impractical and/or unsafe to induce.

\subsection{Distribution of bnAb traits across classes: functional vs. immunological implications for vaccination}

Despite the daunting set of challenges such unusual traits pose, and the emerging evidence these may all be problematic, to varying extents, for the completion of many (most) bnAb pathways, the potentially encouraging news is that matured bnAbs exhibit varying degrees of such features and/or combinations of them, depending on the bnAb "class" they belong 
in, i.e. which epitope clusters in Env they target (Table 1) (Fig 1). For instance, while CD4bs "VRC01 class" bnAbs exceed 40\% SHM levels (Mascola and Haynes, 2013), they have typical sized HCDR3s, and some, like VRC01, lack self-reactivity, both in vitro and in vivo (at least earlier in their development). On the other hand, bnAbs targeting the V2 apex or the high-mannose patch (despite all having long HCDR3s (Burton and Hangartner, 2016)) or those directed to the MPER (despite all thus far exhibiting considerable self-reactivity (Verkoczy, Kelsoe and Haynes, 2014)), tend to accumulate fewer SHMs, relative to CD4bsspecific bnAbs (Bonsignori et al., 2012a; Sok et al., 2013; Verkoczy and Diaz, 2014). The fact that these traits are distributed somewhat differently for lineages targeting different sites in Env, suggests the roadblock (or set of roadblocks) involved in their inefficient development/maturation may be unique to bnAbs targeting distinct common sites, and perhaps also even individual lineages. Below, the distinct skewing of these signature traits based on bnAb class will be discussed, in terms of their functional importance relative to degree of perceived difficulty to induce by vaccination. Two bnAb classes in particular, those directed to the CD4bs, and the V3 high-mannose-targeting patch will be used as notable examples, since the greatest wealth of new precursor opr lineage-targeting immunogens and strategies have been designed or tested in bnAb KI mice thus far for these ones, and thus are most relevant for purposes of discussion for the remainder of this review.

2.2.1 CD4bs-directed bnAbs-CD4-bs-directed bnAbs can be divided into two subclasses, based on how their paratopes interact with CD4bs epitopes. The first group, "HCDR3-binders", bind primarily via contacts in their HCDR3s and includes the CH103 bnAb lineage, while a second group (CD4 mimics via HCDR2) aka "VRC01-class" bnAbs, which include 3BNC60 and VRC01 itself, bind predominantly via HCDR2 contacts, and utilize a canonical gene segment, $\mathrm{V}_{\mathrm{H}} 1-2$ that acts as a structural mimic of CD4 (Scheid et al., 2011; Wu et al., 2010; Zhou et al., 2010).

With respect to the CD4 mimic ("VRCO1-like") bnAb subclass, these have typical HCDR3 regions, unlike most bnAbs, which bear unusually long (and hydrophobic or tyrosine sulfated) HCDR3s. However, these types of bnAbs are not free of carrying unusual Ab combining sites; namely, their LCs while variable, all contain an unusually-short signature 5-amino acid (aa) CDRL3s, a feature that appears to be key for avoiding steric clashes with gp120 (Zhou et al., 2010; Zhou et al., 2015; Zhou et al., 2013). Given the rarity of LCs in the repertoire with this signature, the frequency of VRC01 precursors in individual or Ig locus humanized has been found to be fairly limited (Jardine et al., 2016a; Sok et al., 2016). Perhaps the most prohibitive aspect of VRC01-class bnAbs for vaccination, however, is their exceedingly high SHM levels, more than has been found for any other bnAb class to date (Table 1). While only a subset of these mutations are critical for bnAb function (Georgiev et al., 2014; Jardine et al., 2016b), SHM is nevertheless a largely random process, and AM occurs in non-linear fashion. Thus, eliciting developmental pathways of VRC01-like Abs to maturation will need to induce substantially more mutations than this minimal set, which is still well above estimates of what typical vaccine regimens can induce. Finally, while tolerizing self-reactivity appears to be a universal and profound issue for all MPER Abs tested thus far, either in standard clinical autoantigen assays (Haynes et al., 2005b), host protein arrays (Liu et al., 2015; Yang et al., 2013), or those for which KI models have been 
made thus far ((reviewed in (Verkoczy, Kelsoe and Haynes, 2014)) and (Zhang et al., 2016)), it is encouraging that not all CD4bs are under such early, universal in vivo host controls. For example, a VRC01 HC+LC KI model exhibits normal B-cell development (Tian et al., 2016), although interestingly, a germline 3BNC60 HC+LC Ab KI model is under multiple tolerance controls (McGuire et al., 2016), despite having very similarity immunogenetics (differing only in Vk family LC usage); hence, this suggests LCs may be important in specifying tolerance induction for this bnAb class. Nevertheless, it remains to be seen if VRC01 is also under negative selection, but only later in its maturation, since most matured CD4 mimic bnAbs (including VRC01) avidly bind the conserved host antigen UBE3A ligase, which has been found to have significant overlap with both the VRC01 paratope and CD4 (Liu et al., 2015). The possibility of tolerance induction of VRC01 during AM, will be discussed further below, under the various vaccine studies reported in several independent VRC01 KI systems.

2.2.2 V3-glycan ("N332 supersite)" bnAbs-This class of bnAbs, including the PGT121 clonal family (Mouquet et al., 2012; Walker et al., 2011), and the recently reported DH270 lineage (Bonsignori et al., 2017), target the high mannose patch around the N332 glycosylation site in the V3 loop near the apex of the Env trimer. The epitopes of this class of bnAbs are complex peptido-glycans (Garces et al., 2015; Garces et al., 2014; Mouquet et al., 2012; Walker et al., 2011), and induction of these types of bnAbs faces some of the same hurdles as those for other bnAbs with elongated HCDR3s (i.e. MPER and V2-glycan directed bnAbs), namely, the prohibitively low frequencies their precursors are predicted to be in the human $\mathrm{Ab}$ repertoire, due to multiple pre-immunization issues, either with $\mathrm{HC}$ or SLC pairing (Ivanov et al., 2005; Martin et al., 2003; Vettermann and Jack, 2010; von Boehmer and Melchers, 2010), potential self-reactivity due to their surface expression at the pre-BCR stage (Keenan et al., 2008; Minegishi and Conley, 2001), or at the first tolerance checkpoint upon their expression as IgM+ BCRs (Meffre et al., 2001; Shiokawa et al., 1999). However, the PGT121-clonal family 26 aa long residue is likely critical to penetrate through the glycan layer to interact with the underlying peptides (Pejchal et al., 2010). Other V3glycan class bnAbs do not have as long ( 20aa) HCDR3 regions (Table 1; (Mascola and Haynes, 2013)), including the PGT128 family and the related DH270 lineage; thus these bnAbs may not be as problematic immunologically. The PGT121 and PGT128 clonal family of bnAbs also have other peculiarities that may be difficult for vaccination to induce. In particular, PGT121 has LCs with a 3 aa insertion in framework 3 (LFR3), and PGT128 has a 6 aa insertion in its HCDR2; both events appear important for neutralization breadth/potency (Doores et al., 2015; Pejchal et al., 2011; Sok et al., 2013).

Encouragingly, SHM frequencies for the all V3 glycan bnAbs, although still well above average levels, are lower than most other bnAbs. Perhaps more importantly, intermediates of such bnAbs, for instance, those for PGT121, which already develop considerable breadth, have $~ 10 \%$ SHM (Sok et al., 2013), thus more within ranges reasonable for vaccination. Most remarkably yet, DH270, although not as broad as PGT121, in addition to its relatively shorter HCDR3 noted above, also accumulates considerably less ( $6 \%)$ mutation (Bonsignori et al., 2017), and thus may represent a particularly feasible target for more typical vaccination regimens to elicit. Finally, not much regarding self-reactivity of V3- 
glycan bnAbs and if tolerance control will be a concern for their maturation. In this regard, PGT121 exhibits no overt poly-/autoreactivity in vitro (Liu et al., 2015), and gl-PGT121 HC +LC KI mice exhibit normal development (Escolano et al., 2016), although as will be discussed further later on, it is not known if this knocked-in specificity represent a true intermediate and/or if it bypassed a critical earlier developmental bottleneck. With the molecular elucidation of the complete DH270 lineage (i.e. from bona fide UCA to bnAb), mice expressing either its UCA, or intermediates that have bypassed potential developmental bottlenecks, i.e. one with a G57R substitution, required for breadth in this lineage, are under construction (F. Alt and M. Tian, personal communication), and thus should help clarify this question.

\section{Current gaps in HIV vaccinology are not just knowledge-based, but also technical}

\subsection{The need for more practical animal models}

In addition to the RV144 trial results reported in 2009, in which the first statistically significant, albeit modest, reduction in HIV-1 acquisition by a vaccine regimen were observed (Rerks-Ngarm et al., 2009), the other major milestone in the HIV vaccine development field over the past few years has been the wealth of information obtained from identifying and characterizing the myriad of novel monoclonal bnAbs and their Env targets (Burton and Hangartner, 2016; Burton and Mascola, 2015). This burst in bnAb discovery has largely been enabled by major technological advances on the bnAb discovery side, which as noted above, has involved breakthroughs in memory B-cell sorting, culturing, and $\mathrm{Ab}$ cloning methodologies, as well as increased access to (and more comprehensive longitudinal-sampling of) HIV-infected cohorts. Furthermore, on the bnAb characterization side, equally impressive advances in crystallography, protein modeling technologies, and bio-informatic analyses, have allowed the elegant structural elucidation of the paratopes and epitopes of bnAbs from multiple classes, at near-atomic resolution. However, one area that has lagged behind considerably (until now), has been developing animal vaccine testing models capable of informing the field in an efficient yet relevant way. For such a model to do so, it would need possess a suitable balance of practicality and physiological relevance, allowing it to: 1) conveniently monitor human bnAb activation and maturation in vivo and 2) iteratively test the ability of new HIV vaccine regimens/concepts to induce and broaden $\mathrm{nAb}$ responses.

Access to such practical, yet physiologically relevant, animal models is arguably the largest technical hurdle facing the HIV vaccine field, for two reasons. First, there currently exist many more and increasingly sophisticated vaccine regimens and theoretical concepts, many/ most which cannot be tested in more physiological settings e.g. NHPs and/or vaccine trials, for logistic reasons, i.e. cost, number of experimental groups required. Furthermore, there is a major practical issue facing iterative testing of bnAb-inducing vaccines: that bnAb precursors are expected to be very low in such settings, thus presenting the hurdle of having high enough frequencies to allow for robust, convenient readouts, e.g. where serological analysis is possible (rather than recombinant $\mathrm{Ab}$ cloning). The alternative approach, testing such new vaccine approaches in WT small animal model does overcome some of the logistic 
issues just mentioned, but has the same problem of practicality of readouts, and in addition, fails meet a sufficient standard of physiological relevance, for many reasons that are now becoming more apparent (to be discussed further in Section 3.5), perhaps the most problematic one being that no such small animal models offers a natural alternative that is a truly physiologically-relevant to studying the human Ig repertoire. Such concepts would thus benefit from having a more practical, yet still sufficiently physiological model, capable of bridging the gap between new theoretical concepts and more direct/rapid testing in a relevant setting. The second reason why not having access to practical (yet physiologically-relevant) animal models poses a key technical hurdle for HIV vaccinology is that several key knowledge gaps in the basic B-cell immunology of HIV bnAb responses still exist, which in addition to the issues noted above for "bigger science" iterative vaccine testing, would also profit from such models. For instance, models with the added dimension of genetically malleability, i.e. where cell numbers, B-cell selection environment, and other key factors involved GC and memory B-cell formation can be readily manipulated, would be critical for such, more fundamental questions, in order to more precisely dissect the relevant factors specific to individual bnAbs, limiting and/are minimally required for their vaccine-induced maturation.

In the next three sections, specific knowledge gaps in HIV vaccinology most likely to benefit from improved animal models (having some of the features described above) will be reviewed. Then, in the last section, animal models traditionally used in the field will be compared to various new humanized Ig KI models, either recently generated or under development, which have been engineered to provide the best balance of practicality and physiologic relevance, in order to more feasibly study the atypical biology of bnAb development.

\subsection{Are certain "unusual" bnAb traits functionally needed, but less problematic for vaccination to elicit?}

Given the daunting, yet not impenetrable, set of obstacles facing bnAb induction noted above, what are the most critical steps for developing a bnAb-eliciting vaccine? At least three major knowledge gaps need resolution. The first, which will be reviewed in this section (the others in ensuing sections), is to precisely understand the extent to which "unusual" bnAb traits (or combinations thereof) are required for neutralization breadth, relative to being problematic for vaccination to induce. Importantly, because this knowledge is specifically needed for individual bnAbs, and in the setting of vaccination (rather than infection), more systematic examination across multiple bnAb lineages (representing distinct bnAb classes) is required, in order to obtain a comprehensive picture of all potential bnAb Env responses. Only then can it can be truly ascertained if and which bnAb target(s) are more tractable to elicit. This series of endeavors could thus benefit tremendously from having more practical and physiologically-relevant animal models, that allow development of either singular, or limited sets of bnAb lineages at a much higher resolution to be studies, than currently is possible, due to the rarity of B-cell clones expressing the very traits functionally required for developing bnAb activity. 
Of the normally rare Ab-related traits that typify bnAbs, perhaps the one for which a more fundamental understanding is most needed (and would thus particularly benefit from studies in more practical, and genetically flexible animal models), is the exceptional SHM rates found in all bnAbs isolated thus far (excepting DH270; Table 1). As already noted in Section 1, the levels that bnAb VDJ's accumulate is remarkable (15-48\%) (Burton and Hangartner, 2016; Mascola and Haynes, 2013; Verkoczy and Diaz, 2014; West et al., 2014). Thus, especially critical to ascertain is how much of this SHM is actually required by vaccination to broaden $\mathrm{nAb}$ responses, and if this varies, based on Env region that bnAbs target. In striking contrast to these exceptional levels in bnAbs, Abs from conventional antigenactivated B-cells, i.e. that normally undergo AM in germinal centers (GCs) during typical secondary responses for producing high-affinity Abs (Eisen and Siskind, 1964), exhibit SHM frequencies that plateau at $\sim 6 \%$ (Batista and Neuberger, 1998). Levels above that can lower affinity (Batista and Neuberger, 1998; Kepler and Perelson, 1995; Zhang and Shakhnovich, 2010) and increase chances of creating pathogenic self-reactivity (Mietzner et al., 2008; Shlomchik et al., 1990; Tiller et al., 2007).

The need for these unusually high levels of SHM in bnAbs is currently unknown. However, all bnAbs, thus far, originate from subjects with prolonged HIV-1 infection, where bnAbspecific maturation issues, e.g. extensive host/virus co-evolution (Doria-Rose et al., 2014; Liao et al., 2013b) or potential purifying selection forces (Haynes, Verkoczy and Kelsoe, 2014; Sabouri et al., 2014a; Verkoczy et al., 2013; Verkoczy and Diaz, 2014) could both generate high SHM associated with disfavored, highly-convoluted AM pathways. On the other hand, it is also possible that other, bnAb maturation "independent" confounding issues, i.e. irrelevant to bnAb formation, for example, those related to the chronic infection milieu like inflammation (Moir and Fauci, 2013) or B-cell dysregulation (Levesque et al., 2009), may also contribute high "mutational noise". Additionally, while one study has argued such extreme SHM levels (up to 48\% VDJ aa mutation in VRC01) confer to FRW regions the necessary flexibility to deal with viral diversity (Klein et al., 2013), in vitro mutagenesis studies show only $25-30 \%$ of such high total SHM levels are functionally required (Georgiev et al., 2014). However, this latter report doesn't take into account the non-linear acquisition of SHM accumulated during AM (Batista and Neuberger, 1998). Thus, what component such SHM levels in bnAbs (far higher than in typical Ab responses), is actually needed for vaccination to induce their breath development, is a critical, yet completely unresolved issue.

Based on the initial in vitro mutation studies and accompanying considerations noted above, it is likely that at least some, and likely, considerable amounts of the high bnAb SHM rates are in fact required for their specific maturation process, and thus have direct relevance in the vaccine setting. Assuming this is so, the question then becomes: how much of the specific, yet convoluted AM is functionally driven, versus being host tolerance-induced? In this regard, at least three, non-mutually excusive, bnAb-maturation specific explanations exist for why such high SHM rates are seen (Kelsoe, Verkoczy and Haynes, 2014; Verkoczy and Diaz, 2014) (Fig 2). The first is that these mutations are necessary to modify germline Abs, so as to meet unusually stringent structural requirements (Fig 2, Model "A"). In the case of HIV, due to extensive virus diversification resulting from the co-evolutionary "arms race" between virus and host (Liao et al., 2013b; Moore et al., 2012), such structural 
requirements might not only include high affinity, but also structural flexibility (Klein et al., 2013) to allow for restriction to core epitopes that are poorly recognized by naïve BCRs in the primary, germline Ab repertoire (Haynes et al., 2012b; Hoot et al., 2013; Ota et al., 2012; Xiao et al., 2009) and/or to counter natural variation in otherwise relatively-conserved bnAbtargeting regions (Kwong and Mascola, 2012).

The two other potential "vaccine-relevant" explanations for the unusual SHM levels bnAbs accumulate during prolonged infection, propose that tolerizing self-reactivity, now becoming relevant to limiting development of multiple bnAbs (Chen et al., 2013b; Doyle-Cooper et al., 2013; Finton et al., 2013; Haynes and Verkoczy, 2014; Liu et al., 2015; McGuire et al., 2016; Zhang et al., 2016), is the key driver (Fig 2, Models B and C). One of the two hypotheses by which this occurs (Yang et al., 2013) (Fig 2, Model B) is that due to mimicry of host antigens by many (or most) bnAb epitopes (Haynes et al., 2005b; Kelsoe, Verkoczy and Haynes, 2014), the potential bnAb pool of naïve B-cells, (i.e. with germline (unmutated) BCRs capable of binding self-mimicking bnAb epitopes) are deleted in early development, thus never go on to be recruited into GCs and be driven by AM. In such a "holes-in-the- B cell-repertoire" model, AM is not de novo, as the immune system is forced to work with what is available, and thus recruits weakly cross-reactive, previously mutated B-cell clones that eventually go on to generate bnAb via considerable additional AM. The other, distinct immune tolerance (and HIV mimicry)-based mechanism that has been proposed for driving high SHM levels in bnAbs isolated from infection (Verkoczy et al., 2013) (Fig 2, Model C), is that peripheral tolerance considerably prolongs $\mathrm{AM}$ of bnAb ${ }^{+} \mathrm{B}$-cells, when there exists close (but not complete overlap) in their reactivities for bnAb epitopes and the self-antigens they mimic. In this scenario, mutated, anergic mature bn $\mathrm{Ab}^{+} \mathrm{B}$-cells undergo antigen-driven, "conflicted" purifying selection that acts on V(D)J residues that remove self-reactivity while maintaining affinity for the bnAb epitope. Such a "tug-of-war" process would likely invoke multiple rounds of mutation/selection to de-couple self-reactivity from functional (neutralization) specificity. Attempts at escaping negative selection would result in acquiring many additional mutations that not only remove self-reactivity, but also inadvertently remove bnAb specificity. This process often yields "affinity reversion" byproducts, which normally get deleted (Han et al., 1995; Pulendran et al., 1995; Shokat and Goodnow, 1995). However, in other cases (for reasons as-of-yet not understood), such as those reactive to protective and/or glycan-dependent epitopes, persist as anergic clones in mature B-cell compartments (Sabouri et al., 2014a; Verkoczy et al., 2013), resulting in considerably more SHM, before the final desired "decoupled" end-product of this process is obtained. Additional evidence for this alternative peripheral tolerance process of "affinity reversion/de-maturation", initially proposed as part of a model for GC reaction dynamics (Diaz and Klinman, 2000), has most directly been demonstrated in a well-controlled and elegant set of studies in HEL KI mice (Sabouri et al., 2014a).

Regardless of what the relative contributions of AM-specific (tolerance and/or functionaldriven) or AM independent (i.e. chronic infection-related) issues mentioned above are in producing such high SHM in bnAbs after prolonged natural HIV-1 infection, this issue is nevertheless critical to resolve because if the minimal amount of SHM needed for bnAb generation by vaccination can be defined and the level is considerably less than what occurs during infection, this would provide strong rationale to focus on devising vaccine strategies 
that "short-circuit", rather than recapitulate the evolutionary pathways deciphered during infection, in order to make prime/boost strategies feasible, and thus clinically translatable. Similarly, for the traits of elongated/hydrophobic HCDR3s and in vitro poly/autoreactivity, understanding their in vivo tolerizing effects across bnAb lineages against distinct Env regions is critical, in order to define their tractability as vaccine targets, because immunization strategies can either be directed to lineages with no self-reactivity, or alternatively, focus on modulating tolerance. Such modulation approaches could include regimens that employ appropriate combinations of higher avidity BCR cross-linking, stronger (and/or more specific) Toll-like receptor (TLR) and helper T-cell signals (Chaturvedi, Dorward and Pierce, 2008; Cooke et al., 1994; Lau et al., 2005; Leadbetter et al., 2002; Marshak-Rothstein and Rifkin, 2007; Sekiguchi et al., 2002; Seo et al., 2002) or novel, more specific approaches such as checkpoint-specific, or even molecule-targeting strategies that will be discussed further below, in Section 4.5.

\subsection{Can HIV vaccination guide "reasonable" pathways to breadth at multiple Env targets?}

A second knowledge gap in developing a bnAb-eliciting vaccine, that could also benefit tremendously from having more practical and physiologically-relevant animal models, is understanding whether vaccination can evolve more than one bnAb class/Env target to a degree of breadth that is sufficient for protection, yet feasible for AM to achieve. This would be critical to know because viral escape mutants develop in response to bnAbs targeting only a single region (Liao et al., 2013b; Mascola and Haynes, 2013) and passively infused combinations of two, less-potent/broad bnAbs to distinct targets can synergize in conferring near-complete breadth (Bonsignori et al., 2012a; Bonsignori et al., 2012b; Doria-Rose et al., 2012; Mascola and Haynes, 2013). Taken together, these findings suggest an alternative bnAb-eliciting vaccine strategy, in which cumulative breadth is achieved by eliciting more modest breadth at two or more individual bnAb classes/targets, may not only be more a more reasonable "bar" for vaccine-guided AM to achieve, but may also reduce mutant escape frequency, and thus be at least equally, if not more efficacious. Achieving adequate cumulative breadth would likely also confer additional benefits, including overcoming expected individual $\mathrm{Ab}$ response diversity across the vaccinated population, that could occur in the setting of responses to HIV vaccination, due to numerous factors, including immunogenetic variation, e.g. MHC class II haplotype restriction, or stochastic issues, for example, glycan epitope-targeting bnAbs requiring especially long HCDR3s (PGT121, PG9/16, VRC-26, etc..), resulting in naïve repertoire frequencies so infrequent (likely far lower than VRC01, which has been shown to be rare, i.e. between 1 in a $\sim 1 / 2$ million to 1 in $\sim 2.5$ million (Jardine et al., 2016a; Sok et al., 2016)), that not all individuals may bear relevant lineage predecessors of that particular bnAb class.

Although it remains to be determined empirically, the possibility of pursuing such "cumulative breath" strategies is supported by some potentially encouraging anecdotes. For example, some bnAb intermediates already develop considerable neutralization breadth with more modest SHM levels. In one study, $40-80 \%$ breadth resulted from about $50 \%$ of the total SHM levels observed in the V3/glycan-specific bnAb PGT121 (Sok et al., 2013), which already accumulates relatively less SHM ( $20 \%$ VDJ aa mutation) than many other bnAbs. However, a potential caveat with this more modest estimated overall mutation level of $\sim 10 \%$ 
for achieving modest breadth in vitro is that at least some additional SHM would likely be produced in vivo as intrinsic bystander mutation. However, this particular issue is at least partially countered in theory by the identification and characterization of a new bnAb lineage, DH270, of the same V3/glycan-directed ("N332 supersite") bnAb class, whose matured bnAbs have comparable potency/breadth as PGT121, but whose matured bnAbs accumulates considerably less ( $<5.8 \%$ ) SHM (Bonsignori et al., 2017). Potential identification of other new bnAbs with low SHM, and that target distinct Env sites, would also help to further alleviate such concerns.

However, an additional, and likely more important caveat, even with the identification of such bnAb "outliers" i.e. bearing more reasonable SHM levels, is that we don't know the minimal number of mutations required for vaccination to induce (above and beyond those from in vitro mutational studies), since AM occurs in non-linear fashion, especially in situations where underpinnings of negative selection pressure or conflicted selection (against self, but for neutralization function) exist during maturation, which may occur in many bnAb lineages at some stage of maturation, and thus would still be problematic anywhere such an evolutionary "bottleneck" exists. In other words, even if only a single critical mutation is required for developing breadth and/or even more generally continuing a lineage maturation trajectory, but is one normally under negative selection (that the particular patient from which the original bnAb was isolated, would obviously not have been subjected to), this would likely demand vaccination to induce exceptional AM maneuvers to overcome. A specific example of such high counter-selection on the virus evolution side of bnAb lineage development are mutations capable of recognizing the CD4-proximal N276 residue glycan, which the VRC01 class of CD4bs ${ }^{+}$bnAbs require for neutralization of native Env.

Acquisition of this key glycan, in response to sequential immunization approaches tested thus far in VRC01 KI models (to be discussed further in sections below), appears to be a critical hurdle for bnAb responses, since some maturation to $\mathrm{nAb}$ responses does proceed, but VRC01-like precursors only undergo partial maturation, with serum activity restricted to that capable of neutralizing viruses lacking the N276 glycan (Briney et al., 2016; Tian et al., 2016). A similar potential example of mutation required at only a single critical residue, on the bnAb evolution side of lineage maturation, is the G57R substitution in the IA4 intermediate of the DH270 lineage, a mutation coldspot (i.e. a low-probability site for AID activity), that represents a key event for this bnAb to develop its breadth (Bonsignori et al., 2017). Thus, the question of whether reasonable breath via more achievable vaccine-elicited AM pathways, is achievable at a minimum of two (but preferably more Env targets) is another wide-open question for the field.

\subsection{Emergence of new HIV vaccine formulations and concepts: the need for more iterative testing}

The third key area for bnAb-based vaccine design, that would also benefit immensely from having more practical and robust experimental animal models available, is identifying what forms of HIV immunogens, amongst the myriad being generated, and which combinations thereof, can best induce breadth of nAb responses to HIV-1. Perhaps even more importantly is whether this only partially matters i.e. results in incremental differences, without other critical components i.e. appropriate adjuvanting, T-cell priming, or other vaccine concept. In 
this regard, the area of testing novel, "bnAb germline-targeting" and sequential boosting immunogens is already beginning to markedly benefit from use of such novel testing platforms, in the form of $1^{\text {st }}$ generation "bnAb reverted" KI models (Briney et al., 2016; Escolano et al., 2016), and more recently " $2^{\text {nd }}$ generation" versions of such KI models (Tian et al., 2016). Studies in such models (both will be discussed further in the next section and Section 6), are already providing some initial proof-of-concept (for at least certain bnAb classes), from which potentially key concepts are emerging, for instance, the related ideas that higher affinity priming immunogens are required to activate bnAb predecessors, and sequential administration of at least two (and likely several more) related Envs will be required to guide such bnAb responses appropriately. However, these studies represent only the tip of the iceberg, and given the virtually endless forms and combinations of HIV immunogens currently becoming available to the field, such evaluation, using such practical testing platforms, and versions equally practical but more physiologically relevant for informing vaccine design, will be of paramount importance. What key theoretical questions regarding immunogen design most critically need more comprehensive, robust empiric testing such platforms can afford?

Two of the more fundamental, related questions are: does the form HIV immunogens are in matter in terms of specificity and/or potency of initiating bnAb responses, and does this vary for different Env vaccine targets? At one end of the spectrum, long-standing advocates of polyvalent and/or consensus Env-based immunogens have argued that the presence of multiple Env helper T-cell and B-cell epitopes will be most adept at inducing robust GC responses and AM in as many individuals as is possible. Based on this line of reasoning, incorporating conserved Env epitopes from diverse viral strains would provide global coverage of such responses at a population level (Haynes, 2015). Furthermore, it has been long been suggested that preferentially focusing $\mathrm{Ab}$ responses to those against bnAbs mandate that Env immunogens be in stabilized native trimeric forms, whose structures are believed required to display bnAb epitopes, while occluding many of the more immunodominant non-nAb epitopes, that in natural trimeric Envs would not be accessible for recognition by bnAb ${ }^{+}$B-cells (de Taeye, Moore and Sanders, 2016; Sliepen and Sanders, 2016). However, vaccination with Env trimers on VLP surfaces (Goepfert et al., 2011; Tong et al., 2012) or inactivated HIV-1 virions expressing fusion-active trimeric Envs (Lifson et al., 2002) have thus far still only elicited heterologous, tier 1 nAbs (i.e. Abs that are crossreactive with multiple HIV-1 viral strains having high neutralization sensitivity).

Furthermore, recent studies using near-native soluble recombinant trimers have also resulted in induction of only predominantly tier $1 \mathrm{nAbs}$ (Sanders et al., 2015), although their elicitation of autologous, tier 2 nAbs (Abs reactive to sequence-matched viral strains, having moderate neutralization sensitivity) suggest they may represent a useful starting point. Furthermore, it has also been argued that such immunogens have either not yet been fully optimized (and or iteratively tested) for their ability to optimally activate bnAb precursors (Sliepen et al., 2015), and efforts to increase the affinities for bnAb predecessors by multimerization and/or via other maneuvers, like those being used to optimize native trimeric Envs designed to specifically target V2/glycan bnAb precursors (Andrabi et al., 2015; Gorman et al., 2016) will be discussed below, in Section 5.3. 
On the other end of the spectrum, proponents of "minimal" " immunogens for targeting bnAb lineage responses in vaccine regimens, especially for priming responses(Alam et al., 2017; Alam et al., 2013a; Alam et al., 2011; Hoot et al., 2013; Jardine et al., 2013; McGuire et al., 2013), since most traditional Env immunogens do not bind most precursors of bnAbs at all or only sub-optimally. Furthermore, Env as a complex, multi-epitope immunogens, even when in the most optimal conformation, may still produce significant "off-target" responses, due to expressing irrelevant epitopes, a problem that could be especially accentuated if most bnAb epitopes are anergic and have to compete with irrelevant clones to such epitopes, not under such controls (this issue will also be discussed further in Section 5). However, such minimal immunogens are just beginning to be developed and evaluated, and peptide-based or partial protein subunit vaccine strategies in the past have generally been considerably less successful than protein subunit or whole virus vaccines (Huang et al., 2016; McElrath and Haynes, 2010), possibly because they often lack adequate T helper epitopes. In reality, it is also possible that hybrid approaches may be required. For example, the strength or specificity of T-cell help, even in trimeric Env-based immunization regimens, where quality of T-cell help may be inadequate (due to tolerance controls and/or in some instances, restriction of B and T-cell epitope proximity (Zhang et al., 2014)) could still result in preferential activation of irrelevant non-bnAb responses. Regardless of which types or combinations of immunogens are required in prime/boost strategies, this issue, as with the others mentioned above, would also benefit highly from more comprehensive testing in more practical in vivo platforms.

\subsection{Balancing practicality \& physiologic relevance for HIV vaccine testing: Ig KI vs. other animal models}

All the major knowledge gaps for HIV bnAb vaccine development noted in the three above sections mandate methodical, iterative testing, in order to dissect the roadblocks impeding, and the minimal requirements to developing, bnAb responses to vaccination. This in turn will require more systematic evaluation of individual bnAbs (and their ancestors/maturation branch points) of multiple lineages representative of distinct bnAb classes/Env targets. As already discussed, the most serious limitation in bridging such knowledge gaps (up until very recently) has been a technical one: namely, the lack of appropriate in vivo platforms that allow such questions to be addressed at the required degree of resolution just noted. While no perfect immunization model exists in general, those best suited for this specific task requires the sufficient balance of physiological relevance and practicality (Fig 3). Currently, the only models with this potential are those genetically engineered by mouse $\mathrm{IgH} / \mathrm{L}$ locus-directed targeting of individual (or limited sets of) human pre-rearranged $\mathrm{V}(\mathrm{D}) \mathrm{J}$ exons encoding precursors of matured bnAbs, or alternatively, components of such $\mathrm{V}(\mathrm{D}) \mathrm{J}$ exons (i.e. their unrearranged human $\mathrm{V}$ (D), and $\mathrm{J}$ segments, that in vivo, get developmentally assembled into functional rearranged $\mathrm{V}(\mathrm{D}) \mathrm{J}$ exons). What individual criteria must such bnAb knock in (KI) mouse models fulfill to achieve this unique balance?

On the "practicality" end of the spectrum, bnAb KI mice satisfy two key criteria. First, they represent highly-iterative testing platforms, since by expressing repertoires enriched for bnAb precursors of individual lineages as starting points, they enable studies to be focused on such lineages, where an unparalleled level of resolution and manageable numbers of 
maturation products from numerous trajectories can be rapidly examined and tracked in response to immunization. In contrast, such analysis is not feasible to achieve in human vaccine trials, primate studies, or other wild type (non-genetically modified) animal models with normal polyclonal systems, since individual bnAb roadblocks manifest as a multifactorial set of limitations, resulting in eliciting subdominant bnAb responses, i.e. undetectable by serological-based assays, and thus lack the resolution to more conveniently measure any potential incremental improvements in regimens. The second criteria that bnAb $\mathrm{KI}$ models meet for the required practicality is that they have considerable flexibility: only in such models can B-cell selection, SHM, and GC/Tfh differentiation-specifying processes be genetically manipulated in the setting of focused bnAb lineages, thus allowing even more precise dissection of regulation and development of individual bnAbs. This can be done either by generating compound models via crossbreeding to other relevant, B-cell differentiation process-impacting $\mathrm{KI} / \mathrm{KO}$ strains, or by genetically manipulating timing and/or stage of bnAb expression in the repertoire. The frequency and type of enriched repertoires can also be altered; for instance, they can be derived either from HC only or HC $+\mathrm{LC}$ reverted bnAb rearrangements, preferentially rearranging germline segments, complete humanized Ig loci, or alternatively, as donor B-cells from one (or more) individual reverted bnAb KI model(s), adoptively co-transferred at varying limiting amounts (along with WT repertoire B-cells) into irradiated (or B-cell deficient) recipient mice. All these maneuvers may be critical, at least initially, in order for relevant "signals" of bnAb breadth development to be revealed and/or enhanced during vaccination, since all empirical studies performed to date have yet to yield an effective HIV vaccine, suggesting there exist individual roadblocks in bnAb generation exist that require systematic identification.

On the "physiological relevance" side of the spectrum (Fig 3), bnAb KI models need to serve as pre-clinical vaccine testing platforms capable of complementing human trials and non-human primate studies. Such a complementary role should minimally involve being lead generators for human trials, but ideally, also allow parallel evaluations with trials and/or NHP studies to be made, such that results generated in either setting could be sufficiently informative to the other, that results could be directly translated into subsequent iterations of both studies. In order to enable this kind of testing, such models must have both $\mathrm{Ab}$ repertoires and conserved processes that impact vaccine-mediated induction and maturation of Abs in that repertoire (including bnAb and their various atypical traits), that are sufficiently close to those present in the normal human immune system. These requirements are minimally met by bnAb KI models, due to the way they have been engineered, not only do they bear the relevant human V(D)J exons from which relevant bnAbs are formed, but due to their positioning into the Ig loci, all highly-conserved mechanisms relevant for bnAb activation and development are retained, including (most importantly for bnAb maturation) those driving germinal center (GC) reactions, where high-affinity $\mathrm{Ab}$ formation is orchestrated. Specifically, affinity maturation in GCs is achieved through the combinations of SHM, positive selection for high affinity binding to foreign antigen, and purifying selection (against self-antigen binding), all via nearly identical mechanisms in humans and mice. With further modifications in the way segments are knocked in, for example, as unrearranged $\mathrm{V}, \mathrm{D}$, and $\mathbf{J}$ segments rather than pre-rearranged $\mathrm{V}(\mathrm{D}) \mathrm{J}$ exons, and the frequencies at which they are made can recombine (such newer generation, emerging bnAb KI models 
are under development, and will be discussed in Section 6)), these conditions have the potential to not only be minimally met, but further enhanced to mimicking those in human bnAb development even more closely.

Of note is that the general physiological applicability of the "humanized Ig KI" approach (of which bnAb KI models are a sub-class), for specifically testing bnAb responses to vaccination, differs markedly from that of "humanized" mice, i.e. those reconstituted with human immune systems (reviewed in (Victor Garcia, 2016)(Garcia blanco)), of which perhaps the best characterized and most advanced version is the BLT (ㅁone marrow-LiverThymus) model, i.e. $\mathrm{Rag}^{-/-} \mathrm{\gamma c}^{-/-} \mathrm{CD} 47^{-/}$mice reconstituted with human bone marrow, liver, and thymus) (Melkus et al., 2006). The most notable problem with using such human immune-reconstituted models for vaccine testing (or to understand more fundamental aspects of vaccine guided-bnAb maturation) is that they have generally poor and sporadic $\mathrm{Ab}$ responses (potentially due to incomplete B cell maturation), including weak GC reactions, suboptimal AM; and to varying extents, incomplete CSR. bnAb KI mice are also physiologically superior vaccine models than regular outbred small animals, which not only have the practical issue of polyclonality (noted above), but also have various Ig locus-related immunogenetic features likely problematic for producing a B-cell repertoire capable of evolving the desired human bnAb specificities (reviewed in (Verkoczy and Diaz, 2014)). For instance, mice have both markedly shorter D segments than humans (Schroeder, 2006; Zemlin et al., 2003), restricting their abilities to produce long (>20 aa) HCDR3s, a trait found in $>2 / 3$ of bnAbs (Mascola and Haynes, 2013; Verkoczy and Diaz, 2014).

Additionally, $\sim 50 \%$ of bnAbs use diverse human $\lambda$ LCs which cannot be recapitulated at the WT mouse's atypically-abbreviated $\operatorname{Ig} \lambda$ locus, which in relation to the human $\operatorname{Ig} \lambda$ locus, has restricted diversity and poor expression of $\mathrm{V} \lambda$ segments. Significant differences also exist in $\mathrm{Ab}$ repertoire development between rabbits and humans, including the former using a much more limited set of $\mathrm{V}_{\mathrm{H}}$ segments and a distinct repertoire diversification mechanism, gene conversion (Allegrucci et al., 1991; Knight, 1992), that also pose problems to bnAb generation. By contrast to the various constraints just described for lagomorphs and unmanipulated mice (the two most utilized models in past HIV vaccine studies), any such caveats are circumvented in bnAb KI mice, since, as noted above, such models have been engineered to selectively express human Ig elements required for bnAb development, in place of those in the WT mouse Ig loci (that preclude their development), e.g. knocking-in longer human D segments (or human VDJ exons) at the mouse IgH locus, or human $\mathrm{V} \lambda \mathrm{J} \lambda$ rearrangements at the mouse $\operatorname{IgL}$ locus.

For the remainder of this review, I will discuss the various types of human bnAb KI strains that have been, and are being, developed by my group and others in the field, with the goal of illustrating how, to varying extents, their intended balance of physiological relevance and practicality should allow more focused examination of how B-cell repertoires expressing human bnAbs are formed and selected, and to what extent broad bnAb responses to novel vaccine strategies can develop. I will start with a historical perspective on the use of Ig KI technology to express $\mathrm{V}(\mathrm{D}) \mathrm{J}$ rearrangements of original ( $1^{\text {st }}$ generation) bnAbs, and more recently, " 2 nd generation" and/or "reverted" bnAbs, in order to demonstrate the role of host tolerance in controlling their production (Sections 4.1, 4.2, 4.3), and to elucidate strategies 
for overcoming these roadblocks (Sections 4.4, 4.5, 4.6). I then provide initial insights into vaccine-guided activation and development of bnAb lineages that are starting to emerge from recent immunization studies done with new human Ig KI models that either preferentially express reverted $\mathrm{V}(\mathrm{D}) \mathrm{J}$ rearrangements or fully germline (unrearranged) $\mathrm{V}$ gene segments of $2^{\text {nd }}$ generation bnAbs (Section 5). Finally, I will review technical advances for higher-throughput production of, or conditional expression in, bnAb KI models, to further accelerate basic studies of vaccine-guided bnAb development and regulation (Section 6). Ultimately, I project how such methodological improvements will make testing of individual bnAb lineages to distinct targets (and/or key members of such lineages) a more systematic exercise, thus making more feasible two critical goals for HIV vaccine development: defining the key parameters required for vaccine-guided bnAb induction and identifying the lineage-directed immunogens and prime/boost strategies can best do this.

\section{Human Ig KI models in elucidating bnAb tolerance controls and testing ways to overcome them}

Negative selection of B-cells does not occur as a single discrete process, but encompasses a continuum of complex and related phenomena. Indeed, the various effects of immune tolerance on B-cell development are driven by various factors during encounter of selfantigens with B-cells, including the form in which self-antigens are presented (Hartley et al., 1991), the affinity at which they bind (Chen et al., 1995b; Li et al., 2001), the location/site of their interactions with B-cells (Murakami et al., 1992; Russell et al., 1991), and the stage of B-cell maturation at which this encounter occurs (Benschop et al., 2001). Furthermore, negative selection of B-cells occurs at several checkpoints during their ontogeny (Wardemann et al., 2003) and by various mechanisms that can impact B-cell frequency, BCR signaling responsiveness, and/or BCR specificity (reviewed in (Goodnow et al., 2010; Shlomchik, 2008). Since the late 1980's, Ig transgenic models in which B-cells specific to defined self-antigens, have been engineered from single pre-rearranged specificities $\left(\mathrm{V}_{\mathrm{H}} \mathrm{DJ}_{\mathrm{H}}\right.$ and/or $\mathrm{V}_{\mathrm{L}} \mathrm{J}_{\mathrm{L}}$ rearrangements) either randomly integrated into the genome (i.e. conventional Ig transgenic models), or relatively more recently, knocked-into the IgH and L loci (i.e. sitedirected Ig transgenic, aka knock-in (KI) models), have been invaluable in providing the resolution required to systematically dissect negative selection processes (reviewed in (Goodnow, 1992; Kumar and Mohan, 2008)). These Ig tg/KI-elucidated mechanisms include central deletion (Erikson et al., 1991; Hartley et al., 1991; Nemazee and Burki, 1989; Okamoto et al., 1992), LC receptor editing (Gay et al., 1993; Tiegs, Russell and Nemazee, 1993), $V_{H}$ replacement (Chen et al., 1995a), peripheral anergy (Goodnow et al., 1988; Goodnow et al., 1989), follicular exclusion (Cyster, Hartley and Goodnow, 1994) and splenic marginal zone or peritoneal sequestration (Hayakawa et al., 2003; Li, Li and Weigert, 2002; Murakami et al., 1992). What is also clear in these various tg/KI systems is that tolerance is manifested in a gradient of "severity", that has taught us many of the principles of what factors influence in vivo tolerization outcomes, as well as the concept that not all Abs predicted to be auto-Abs by clinical definitions have tolerizing effects in vivo. Below, I will discuss how Ig KI mice, akin to these classic models, but specifically made to express $\mathrm{V}(\mathrm{D}) \mathrm{J}$ rearrangements of several prototypical bnAbs, have been used to formally demonstrate if, and dissect how, negative selection mechanisms limit their production, and 
more recently, how such models have now become the accepted standard to evaluate the potential role of such processes for newer bnAbs, with several KI models expressing such " $2^{\text {nd }}$ generation" bnAbs (or their precursors/lineage ancestors) now having been developed and currently under analysis, both in the presence or absence of vaccination.

\subsection{Initial work in KI mice demonstrating negative selection of MPER+ bnAb-expressing B- cells}

Prior to 2009, only a handful of monoclonal bnAbs (2F5, 4E10, 2G12, b12 and Z13e) had been identified. Of these " 1 st generation" bnAbs, only three (2F5, 4E10, and 2G12) expressed naturally-derived $\mathrm{HC} / \mathrm{LC}$ pairs by virtue of having been directly isolated from chronically HIV-1 infected subject peripheral blood mononuclear cells (PBMCs) using classical hybridoma technology, whereas the other two were obtained from phage display libraries of randomly-recombineered Abs. Of these three early bnAbs obtained by hybridoma cloning, both 2F5 and 4E10, directed to adjacent linear epitopes in the gp41 MPER, were reported to exhibit traits that represent strong predictors of negative B-cell selection: significantly elongated and hydrophobic HCDR3s, and in vitro polyreactivity and/or autoreactivity, as determined by immunohistochemical staining of HEp-2 cells and a standard clinically-defined autoantigen panel, respectively (Haynes et al., 2005a). This led to the hypothesis that induction of bnAb responses was impaired by B-cell tolerance mechanisms (Haynes et al., 2005b). Analogous to the approaches previously used to test in vivo tolerization of KI B-cells reactive to DNA (Chen et al., 1995b; Erikson et al., 1991), erythrocytes (Murakami et al., 1992; Okamoto et al., 1992), or to artificially-introduced selfantigens-created via dual KI/tg systems, such as the a-MHC Ig tg/KI+MHC 3-83 tg (Braun, Rajewsky and Pelanda, 2000; Nemazee and Burki, 1989) or a-hen egg lysozyme (HEL) Ig tg/KI+HEL tg models (Hartley et al., 1991), evidence supporting this hypothesis was first demonstrated in $2 \mathrm{~F} 5 \mathrm{~V}_{\mathrm{H}} \mathrm{DJ} \mathrm{H}_{\mathrm{H}} \mathrm{KI}$ mice exhibited, which exhibited profound deletion ( 95\%) of B cells expressing BCRs in bone marrow and a significant majority of the remaining B cells in the periphery were anergic (Verkoczy et al., 2010). Subsequently, three independent groups expressed the original (mutated) $\mathrm{V}_{\mathrm{H}} \mathrm{DJ}_{\mathrm{H}}$ and $\mathrm{V}_{\mathrm{H}} \mathrm{DJ}_{\mathrm{H}}+\mathrm{V}_{\mathrm{L}} \mathrm{J}_{\mathrm{L}}$ rearrangements of $2 \mathrm{~F} 5$ and/or 4E10 (Chen et al., 2013b; Doyle-Cooper et al., 2013; Finton et al., 2013; Verkoczy et al., 2011a; Verkoczy et al., 2010) and remarkably, all 2F5/4E10 KI models generated had very similar profound blockades at the pre-B to immature B-cell transition in the bone marrow, characteristic of clonal deletion at the first tolerance checkpoint, and similar to those seen in Ig KI/tg mice expressing high affinity and/or membrane-bound self-antigens (Chen et al., 1995b; Erikson et al., 1991; Hartley et al., 1991; Nemazee and Burki, 1989). The CD4bs-specific monoclonal bnAb b12, the other " 1 st generation" bnAb reported to exhibit in vitro poly/autoreactivity (Haynes et al., 2005a), was found to lack any obvious in vivo tolerizing self-reactivity in KI mice expressing its $\mathrm{V}(\mathrm{D}) \mathrm{J}$ rearrangements (Ota et al., 2013), although the physiologic significance of this finding currently remains ambiguous, since b12's origin from a phage library makes it unclear if a bona fide comparable HC/LC pairing exists in vivo, and additionally, detailed molecular or functional signaling analysis of the peripheral B-cell subsets in these mice was not reported. Nevertheless, this finding does raise the issue of drawing premature conclusions regarding how bnAbs may be controlled by negative selection in vivo, based strictly on readouts of standard in vitro poly-/autoreactivity assays, an issue that will be revisited further in several parts of this review. 


\subsection{Central deletion and other B-cell tolerance mechanisms observed in bnAb KI models}

Despite the stringency of central deletion observed in 2F5 and 4E10 VDJ+VJ KI mice, it was not found to be complete, as small populations of residual KI B-cells populate the periphery of both models, which could serve as targets for immunization. These residual KI B-cells, however, were found to be limited by additional, $2^{\circ}$ tolerance mechanisms, including having poor BCR expression and signaling, characteristic of being anergic (functionally-silenced) (Cambier et al., 2007), and/or undergoing extensive LC receptor editing events that mitigated self-reactivity and promoted rescue from clonal deletion (Chen et al., 2013b; Doyle-Cooper et al., 2013; Verkoczy et al., 2011a). Notably, many peripheral clones remained anergic, despite having undergone an extensive degree of LC editing (Chen et al., 2013b; Verkoczy et al., 2011a), which along with the finding that $\mathrm{V}_{\mathrm{H}} \mathrm{DJ}_{\mathrm{H}}$, i.e. " $\mathrm{HC}$ only" versions of these KI models, in which only $\mathrm{V}_{\mathrm{H}} \mathrm{DJ}_{\mathrm{H}}$ rearrangements are expressed and thus free to pair with the entire mouse LC repertoire, still exhibited profound deletion and anergy tolerance controls (Chen et al., 2013b; Doyle-Cooper et al., 2013; Finton et al., 2013; Verkoczy et al., 2011a; Verkoczy et al., 2010), suggested that the 2F5/4E10 HC selfreactivities are dominant and are not efficiently vetoed by LC editing.

In terms of further understanding the tolerance mechanisms controlling bnAb induction, the 2F5 VDJ+VJ strain, as the prototype bnAb KI tolerance model, has been the most extensively studied thus far (Fig 4), and as such has provided some additional insight into these processes. Specifically, in addition to the processes of deletion, anergy, and receptor editing noted above, further studies have also led to identification of a novel process termed Affinity Reversion/Ab redemption that represents a "last resort" mechanism for anergic $2 \mathrm{F5}^{+} \mathrm{B}$-cells that persist into mature B-cell subsets (including the aforementioned "uneditable" anergic population) for escaping apoptosis via purifying selection (Verkoczy et al., 2013). The same phenomenon has independently been uncovered and elegantly described in a well-controlled set of HEL KI models by Goodnow and colleagues (Sabouri et al., 2014a). Recently, evidence for this process in limiting completion of bnAb maturation has been observed in the development of 2F5-like lineages in vaccinated primates (Zhang et al., 2016). Based on these emerging data in support of this process, one hypothesis to account for the high degree of SHM in bnAbs has been proposed, wherein the incomplete overlap of bnAb epitopes with self-antigens creates a selection "tug-of-war" during AM: for HIV neutralization affinity, but against self (Haynes, Verkoczy and Kelsoe, 2014; Kelsoe, Verkoczy and Haynes, 2014; Verkoczy and Diaz, 2014). Since bnAbs appear to utilize tortuous AM pathways to develop breadth during their co-evolution with HIV in chronicallyinfected subjects (Mascola and Haynes, 2013), it will now be of interest to explore the relevance of Affinity Reversion during vaccine approaches for eliciting various bnAb lineages, since such a mechanism, if more universally relevant, would have obvious conceptual relevance in terms of guiding novel immunogen design approaches. In such cases, "escape clones" that have de-coupled Env and self-reactivities, i.e. with neutralizing potential, but lacking self-binding, could be identified and used to map Env mutants retaining only residues critical for function, and subsequently used as boosting immunogens, to drive B-cells, once they have been appropriately induced by priming to overcome anergy. 
From these studies in 4E10/2F5 KI models, three key, related questions have arisen: 1) do tolerance controls limit induction of all MPER ${ }^{+}$bnAbs? 2) to what extent do they control responses of bnAbs targeting other Env regions? And 3) are they also operational in bnAb precursors and/or intermediates, which as such, at least partially contribute to high SHM levels seen in fully-matured bnAbs? Understanding tolerance prevalence across bnAb lineages to distinct Env regions now becomes a critical question for the field, because obtaining such knowledge will be a critical component in defining their relative tractability as potential vaccine targets, specifically with respect to informing as to whether vaccine strategies should either be directed to lineages with no self-reactivity, or alternatively, be focused on modulating tolerance. Based on the studies done performed in the " $11^{\text {st }}$ generation" 2F5/4E10/b12 bnAb KI lines, it is clear such models represent the "gold standard" to now definitively test if and how in vitro self-reactivity physiologically impacts development of " $2^{\text {nd }}$ generation" bnAbs (bnAbs with more potency and/or breadth, and identified in 2009 onwards), and their precursors (before or during immunization), for two reasons. First, as alluded to in the previous section, the presence or absence of bnAb selfreactivity in vitro is often, but not always a predictor of their in vivo tolerization (reviewed in (Verkoczy and Diaz, 2014)). For instance, false negatives can arise due to self-antigens having restricted expression or affinities sufficient to trigger deletion in vivo (Lang et al., 1996) but below detection in vitro. Conversely, false positives may occur when in vitro reactivities to self-antigens don't reflect those capable of invoking tolerance at physiologically relevant sites of B-cell encounter. Secondly, because host tolerance occurs in a continuum of developmental checkpoints and processes (Kelsoe, Verkoczy and Haynes, 2014; Verkoczy et al., 2011b) only through analysis via their expression in the setting of Ig $\mathrm{KI}$ models can precise mechanisms responsible for limiting bnAb production and most critically, ways to manipulate such processes, be identified, as has been previously done for examining the developmental fates of various auto-Ab specificities.

\subsection{Further evidence of host controls in newer bnAb precursor KI strains, and in other recent studies}

Although potential in vivo tolerization has only been evaluated for many of the $2^{\text {nd }}$ generation bnAbs or their lineage members, several initial insights have begun to emerge from studies in recently produced KI models expressing "germline-reverted" or unmutated common ancestor (UCA) rearrangements from some of these newer bnAbs. One such example is a recent study in which mice expressing $2 \mathrm{~F} 5$ 's unmutated ancestor (UA) $\mathrm{V}_{\mathrm{H}} \mathrm{DJ}_{\mathrm{H}}$ $+\mathrm{V} \kappa \mathrm{J} \kappa$ rearrangements were generated, and found to be under more profound deletion and anergy controls relative to the original (mutated) 2F5 dKI mice (Zhang et al., 2016), demonstrating in certain instances, high SHM levels in bnAbs can accumulate at least in part due to elimination of self-reactivity. In this particular case, the accumulation of SHM correlated and relative lowering of in vivo tolerization of this bnAb and its UA precursor could potentially be explained by AM to breadth development having focused this bnAb precursor's specificity from one of high initial in vitro polyreactivity/higher lipid affinity (Alam et al., 2011) to specificity for only one (or a few) self-antigen(s). A second recent example where the impact of expressing a $2^{\text {nd }}$ generation bnAb precursor on $\mathrm{B}$-cell development has been evaluated is a study in which a gl-3BNC60 VDJ+VJ KI model, coexpressing $\mathrm{V}$ germline-reverted $\mathrm{V}_{\mathrm{H}} \mathrm{DJ}_{\mathrm{H}}$ and $\mathrm{V}_{\mathrm{L}} \mathrm{J}_{\mathrm{L}}$ rearrangements of $3 \mathrm{BNC60}$, a "CD4 
mimic" VRC01-class CD4bs+ bnAb (McGuire et al., 2016). In this strain, KI B-cells were partially deleted in the bone marrow, and peripheral cells were either anergic or exhibited extensive editing of their gl-3BNC60 VJ rearrangement via secondary rearrangements of their $\lambda$ LC locus. Most recently, the effect of expressing another $2^{\text {nd }}$ generation bnAb precursor, isolated from a "HCDR3-binder" CD4bs+ bnAb lineage, CH103, on B-cell development was evaluated (W. Williams, B. Haynes, and L. Verkoczy, manuscript

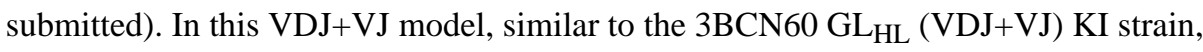
partial clonal deletion was observed, but with this particular bnAb specificity, occurred at the $2^{\text {nd }}$ tolerance checkpoint (i.e. the transitional to mature B-cell stage), and the mature B-cell subset was predominantly comprised of naïve B-cells that had undergone receptor editing of their knocked-in UCA CH013 LCs. Interestingly, the fraction of remaining clones that did manage to retain both their $\mathrm{HC}+\mathrm{LC} \mathrm{KI}$ alleles (were anergic), represented a very minor subset, $<10$ and $0.5 \%$ of the mature subset in homozygous and heterozygous versions of this strain, respectively, suggesting LC editing was the major tolerance mechanism in this model. Interestingly, LC editing also appeared to be highly restricted (predominantly to a single V $\mathrm{K}$ family), reminiscent of the medium-affinity version within the $3 \mathrm{H} 9$ anti-DNA series of $\mathrm{Ig} \mathrm{KI}$ models, 3H9/56R (Li et al., 2001; Li, Li and Weigert, 2002), suggesting that while editing could veto $\mathrm{CH} 103$ self-reactivity, it could only do so with a highly restricted set of "LC editors".

In contrast to these two reports, $\mathrm{V}_{\mathrm{H}} 1-2 / \mathrm{KI}$ mice expressing the unrearranged germline segment $\mathrm{V}_{\mathrm{H}} 1-2$, found in all members of the VRC01 bnAb class, along with the VRC01 germline $V_{L} J_{L}$ rearrangement did not have any defects in B-cell development, but when a robust vaccine regimen, tailored for optimal priming and with a rational sequential boosting strategy (discussed in detail, further below), only partial maturation of VRC01-like precursors could be induced, with serum neutralization activity of mutant viruses (lacking key CD4bs-proximal glycans), but not of WT (glycan-containing) ones (Tian et al., 2016). Since the VRC01 antibody, although lacking polyreactivity in protein-arrays, has high avidity for the conserved ligase UBE3A, like several other CD4bs ${ }^{+}$bnAbs (Liu et al., 2015), it remains to be seen if this vaccine-guided maturation blockade is a result of negative selection (possibly UBE3A-mediated) in the GC reaction. Interestingly, the VRC01 and 3BNC60 lines express the same $\mathrm{V}_{\mathrm{H}^{1}} 1-2$ segment as well as the same 5aa-LCDR3 residues, despite their marked pre-immune developmental differences (i.e. precursor VRC01 with normal B-cell development and precursor 3BNC60 exhibiting deletion, anergy, and editing),

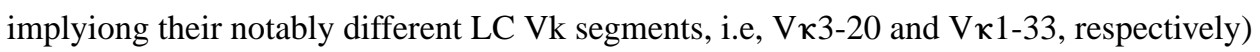
may specify these distinct developmental/selection fates. Nevertheless, more generally, these data provide evidence that the processes of B-cell elimination, silencing, or modification apply not only for all MPER ${ }^{+}$bnAbs tested thus far in vivo, but also most (if not potentially $\underline{\text { all) }}$ predecessors of $\mathrm{CD}_{4} \mathrm{bs}^{+}$bnAbs evaluated to date, are under varying degrees of these controls, at some point in their development, either before or during vaccination.

Equally important developments have also emerged from two key new studies performed in settings other than KI models, which further support findings of considerable tolerance prevalence across bnAb lineages, thus complementing the above-mentioned initial studies in these newer (non-MPER bnAb) KI models. In one of these studies, a protein array of $~ 9400$ host proteins was used to screen a large array of HIV Abs (including many $2^{\text {nd }}$ generation 
bnAbs directed to the four major Env epitope clusters) for off-target binding, and found $>2 / 3$ were poly- and/or autoreactive (Liu et al., 2015), including two of the most potent and broad ones, the MPER ${ }^{+}$bnAb 10E8 and the CD4bs specific bnAb VRC01, both for which selfreactivity was not originally detected in standard in vitro assays (Huang et al., 2012; Wu et al., 2010; Zhou et al., 2010). Importantly, only one non-neutralizing HIV Ab tested in this assay exhibited polyreactivity, suggesting poly- and/or autoreactivity is a property of bnAb functional specificity rather than a byproduct of the inflammatory/dysregulation milieu that typifies longer-term chronic HIV-1 infection (Haynes et al., 2011; Moir and Fauci, 2013). Also consistent with host controls limiting many (or most) bnAbs is a new study providing direct evidence in support of the corollary that bnAbs should be more easily generated in autoimmune patients (due to defective central and/or peripheral tolerance checkpoints), a concept that is consistent with anecdotal observations of disproportionately low frequencies of $\mathrm{SLE}^{+}$patients also having HIV infection (Barthel and Wallace, 1993; Calza et al., 2003; Kaye, 1989; Mylonakis et al., 2000; Palacios et al., 2002). In this new, comprehensive study, two HIV-infected cohorts differing in their abilities to produce bnAbs were compared, and relative to matched subjects that failed to generate bnAb responses, those making bnAbs had significantly higher frequencies of circulating plasma auto-Abs, higher numbers of $\mathrm{CD} 4^{+} \mathrm{T}$ follicular helper cells and lower numbers of T regulatory cells, the latter which also have higher levels PD-1 expression that has also been associated with impaired regulatory capacity (Moody et al., 2016).

Thus, considerable data is mounting for the role of $\mathrm{B}$-cell tolerance in limiting bn $\mathrm{Ab}$ development, and taken together with the above recent cohort comparison study, points to a rational path forward for vaccine development that not only considers immunogens capable of recreating (and likely needing to improve on) the viral events occurring during natural infection, but adjuvant formulations that can also simulate the relevant immune perturbations identified in some individuals during chronic infection. In the two sections below, some early studies in bnAb KI mice and other animal models, aimed at overcoming negative Bcell selection in order to enhance sub-optimal (b)nAb responses to HIV-1, will first be reviewed, followed by discussing newer tolerance checkpoint inhibition strategies, either using mAbs and/or small molecule inhibitors under consideration for carrying such types of studies in a more directed fashion.

\section{4 $\mathrm{KI}$ mice as models to identify strategies to break bnAb anergy}

As just discussed above, from accumulating data in multiple bnAb KI models, it appears many, if not most bNAb lineages (even some found initially to lack in vitro polyautoreactivity, e.g. 3BNC60) will be limited by the various B-cell tolerance controls at some point in their evolution. Assuming tolerizing self-reactivity of bnAbs is indeed a prevalent issue (i.e. present in bnAbs against multiple Env targets, and/or at key branchpoints in common maturation pathways of many lineages), then several major questions now emerge for understanding how to induce bnAbs. Two key, related ones are: 1) can bnAb selfreactivity can be overcome by vaccine strategies? and 2) if so, can inducing them by vaccination be done safely? With respect to the first question, a common theme emerging from studies in 2F5 UA, gl-3BCN60 VDJ+VJ KI, and now, CH103 UCA VDJ+VJ mice, is that although B-cells that express bnAb precursors as BCRs appear to often be tolerized 
even prior to vaccine-driven maturation (McGuire et al., 2016; Zhang et al., 2016), it is nevertheless encouraging that not all such B cells are culled early in development by clonal deletion. This results in residual anergic $\mathrm{KI}^{+} \mathrm{B}$-cell populations that persist in peripheral tissues at various developmental stages, and thus are available for vaccine regimens to target/re-activate. However, this is not trivial to do, since anergic B-cell are preferentially selected against by immunization-mediated exclusion (Cyster, Hartley and Goodnow, 1994). Furthermore, it is now becoming better appreciated that B-cell anergy itself, does not manifest as a single discrete phenotype, both in terms of stages at which it can potentially occur and the phenotypic and functional heterogeneity of the B-cells it functionally silences (Cambier et al., 2007). Thus, the potential reversibility of BCR unresponsiveness of various anergic B-cells populations to immunization may vary. Finally, reversing anergy does not preclude re-activated self-reactive B-cells from having their self-mimicking Env specificity eliminated by some of the $\mathrm{V}(\mathrm{D}) \mathrm{J}$-modifying processes discussed above, and thus, depending on the stage in B-cell development at which anergy is broken, this can involve preferential selection for clones bearing BCRs that either have undergone receptor editing (DoyleCooper et al., 2013; Verkoczy et al., 2011a; Zhang et al., 2016), and/or in the case of those in mature B-cell compartments specifically, have been subjected to purifying selection via SHM-targeted elimination of self-antigen binding Ab residues, i.e. redemption/affinity reversion (Sabouri et al., 2014b; Verkoczy et al., 2013).

Previous studies examining signals required to overcome B-cell unresponsiveness in the prototypical series of a-hen egg lysozyme (HEL)+soluble HEL anergy KI/transgenic models have shown that addition of stronger or more specific T-cell help (Sekiguchi et al., 2002; Seo et al., 2002) and/or higher avidity (multimeric) BCR signals (Cooke et al., 1994; Goodnow, Brink and Adams, 1991) to enhance B-cell survival (in adoptive transfer settings where such signals are normally limiting), can re-activate anergic B-cells to secrete class-switched Abs, and other studies have similarly shown that BCR/TLR co-ligation can trigger residual anti$\mathrm{DNA}^{+}$self-reactive peripheral B-cells (Chaturvedi, Dorward and Pierce, 2008; Lau et al., 2005; Leadbetter et al., 2002; Marshak-Rothstein and Rifkin, 2007). Interestingly, two recent studies performed in wild type mice have demonstrated that overall anti-HIV Ab responses can be enhanced, when Env immunizations are carried out in the presence of cytokines capable of providing strong co-stimulatory signals and/or enhanced peripheral B-cell survival (Dosenovic et al., 2012; Gupta et al., 2015). In one of these studies, preadministration with BAFF/BlyS resulted in increased frequencies of self-reactive B-cells that was accompanied by increased potencies of Tier 1 serum nAb responses to Env gp120 immunization (Dosenovic et al., 2012). In the other study, DNA vectors expressing multimerized BAFF or APRIL, when added to an Env gp140 + IL-12 immunization regimen, induced more robust GCs and modestly enhanced autologous Tier $1 \& 2$ serum nAb titers (Gupta et al., 2015). However, due to the limitations of performing immunization studies in wild type mice I discussed earlier, related both to the restrictions imparted on potential bnAb responses by murine immunogenetics and the current sub-dominance of serum bnAb responses in fully polyclonal settings, including the Env specificities of $\mathrm{Ab}$ responses in these studies were cumulative and variable, it could not be ascertained how individual bnAb lineages were initiated by such modified vaccine regimens, if at all. 
In bnAb KI studies, where it is possible to evaluate specific effects of anergy-targeting vaccine regimens on the development of individual bnAb lineages, results have begun to provide proof-of-principle that self-reactivity of bnAbs (or their precursors) can indeed be overcome by immunization. In gl-3BCN60 VDJ+VJ KI mice, McGuire et al. found that anergic $\mathrm{KI}^{+}$cells could be re-activated, but only when $426 \mathrm{c}$.TM4 $4 \mathrm{~V} 1-3$, a soluble GLtargeting gp140 immunogen, was highly multimerized (McGuire et al., 2016), thus recapitulating previous findings of the experimental anergy models, such as in various antiHEL tg/KI strains, in which particulate HEL antigens can re-activate anergic B-cells that soluble HEL failed in doing so (Goodnow, Brink and Adams, 1991; Sabouri et al., 2014a). However, even with this modification, the gl-3BNC60 V(D)J rearrangements underwent almost no SHM. Likewise, in 2F5 UA VDJ+VJ KI mice, Zhang et al. demonstrated that a residual anergic $\mathrm{KI}^{+} \mathrm{B}$-cell subset could be targeted for expansion/activation by a vaccine regimen comprised of MPER peptides, when conjugated to liposomes and formulated with TLR agonists, but these expanded clones underwent minimal immunoglobulin class switching (CSR) and SHM (Zhang et al., 2016). This raises the possibility that in both models, additional signal(s) may be required to activate a T-dependent response. Interestingly, Verkoczy et al. previously found this same MPER peptide/liposome/TLR agonist regimen was also capable of inducing residual anergic $\mathrm{dKI}^{+}$clones in original (matured) 2F5 VDJ+VJ mice to produce high $(\mathrm{mg} / \mathrm{ml}$ ) titers of serum bnAb IgGs (Fig 4) (Verkoczy et al., 2013). However, unlike 2F5 UA KI ${ }^{+}$cells, they were able to undergo class switching (possibly due to being under less profound tolerance controls (Zhang et al., 2016) and/or the mature 2F5 Ab having higher affinity for the MPER epitope than the 2F5 UA Ab (Alam et al., 2011)). It is noteworthy that despite the considerable class-switching observed by vaccine-mediated re-activation of $2 \mathrm{~F} 5$ mature $\mathrm{KI}^{+}$anergic $\mathrm{B}$-cells, this was genetically demonstrated to occur via a T-independent pathway (Verkoczy et al., 2013), suggesting T cell help and/or BCR signals were insufficient for activating $2 \mathrm{~F} 5$ mature $\mathrm{dKI}^{+} \mathrm{B}$-cells, and to an even greater degree, $2 \mathrm{~F} 5 \mathrm{UA} \mathrm{dKI}^{+} \mathrm{B}$-cells. Notably, while this earlier study also found that both presentation of the MPER 2F5 bnAb epitope in liposomes and TLR adjuvanting were critical to break anergy (Verkoczy et al., 2013), it did not ascertain if this was due to antigen multimerization in combination with TLR co-ligation (thus working as a classic Type $2 \mathrm{~T}$-independent immunogen), or if more specific components in the regimen were involved. One clue supporting the latter possibility has come from follow-up studies that further dissected the regimen, in which specific presentation of the MPER peptide in liposomes, i.e.in an orientation designed to mimic the epitopes as they would be expressed on HIV-1 virions (in contrast to general multimeric display), and only with the TLR-4 agonist monophosphoryl lipid A (versus other TLR agonists tested), were both key to reverse the anergic state of peripheral $2 \mathrm{~F}^{2} \mathrm{dKI}^{+} \mathrm{B}$-cells and for the induction of high serum bnAb IgG titers in 2F5 and 4E10 VDJ+VJ mice ((Chen et al., 2013a); H. Bouton-Verville, L. Verkoczy et al., manuscript in preparation), suggesting a key, specific role for lipid components in overcoming anergy of $\mathrm{MPER}^{+}$bnAbs. An intriguing remaining question regarding overcoming MPER bnAb anergy is whether strength of $\mathrm{T}_{\mathrm{H}}$ priming and degree of tolerization are related (i.e. is stronger and/or more specific $\mathrm{T}$ cell help required to fully reactivate and drive maturation of more profoundly anergic bnAb precursors?), an issue that can now be rigorously examined via parallel evaluation of priming strategies in mature 2F5 $\mathrm{KI}$ and 2F5 UA KI models. More generally, it will also be of interest to understand if signals 
for triggering anergic $\mathrm{KI}$ cells in the gl-3BCN60 and 2F5 UA KI models are similar to those potentially needed to re-activate bnAb precursors targeting other Env regions.

\subsection{Testing more targeted tolerance checkpoint modulation approaches in bnAb KI mice}

\subsubsection{Modulation of peripheral bnAb tolerance using CD4 T-cell inhibition}

therapy-Many of the same genes and molecular signaling pathways that control the negative selection checkpoints involved in vetting self-reactive B-cells (Figs 4,5) including clones expressing bnAbs (and/or their predecessors), have considerable overlap with those involved in tumor suppression (reviewed in (Goodnow, 2007)). Since many drugs targeting checkpoint survival genes and differentiation pathways have been developed by the cancer field in the clinical setting, then therapies involving inhibitors of such checkpoints, by extension, could also be available for use to more transiently modulating distinct B-cell tolerance checkpoints that prevent bnAb maturation and/or, either prior or during HIV immunization. Many of these more successful approaches tried thus far for cancer (i.e. which have resulted in significant clinical advances, and in many patients has elicited long term clinical benefits) are immune checkpoint therapies that target regulatory pathways limiting T-cell proliferation and enhanced immune responses to malignancies (Sharma and Allison, 2015a; Sharma and Allison, 2015b), and as such, are most relevant for peripheral Bcell tolerance checkpoints in particular. Following the lead of these more effective cancer treatments, HIV-1 vaccine immunogens using such treatment regimens, either before or during immunizations, have therefore now begun to be carried out in both macaques, and in models with more pronounced, selective tolerization later at the $2^{\text {nd }}$ checkpoint (transitional to mature B-cell stage) and/or even later during the GC reaction, e.g. CH103 UCA dKI and gl-3BNC60HJ (VDJ+VJ) mice would be good initial readouts for testing such approaches (G Kelsoe, B. Haynes, and T. Bradley, personal communication, \& in collaboration with L. Verkoczy). Examples of such "modified" HIV-1 regimens include those using Abs previously used in cancer therapeutics, namely Abs against CTLA-4, the programmed death-1 receptor, i.e. PD-1, and the IL-2 receptor a chain, i.e. CD25 (Belkaid and Tarbell, 2009; Chambers et al., 2001; Sharma and Allison, 2015a; Sharma and Allison, 2015b). Although both functionally complicated, CTLA-4 and PD-1 are believed to have nonoverlapping modes of action: while CTLA-4 is generally thought to inhibit co-stimulation (Chambers et al., 2001; Teft, Kirchhof and Madrenas, 2006), PD-1 works by differentially interfering with signals mediated through the $\mathrm{T}$ cell receptors (TCR) which on conventional T-cells, results in delivery of inhibitory signals, while on $\mathrm{T}_{\text {regs }}$, enhance proliferation and survival (Keir et al., 2008; Sharpe et al., 2007). Thus, if the main goal of bnAb-potentiating vaccine strategies is to increase survival of peripherally tolerized mature follicular B-cells, then one class of regimens appropriate for doing so could be ones that include anti-CTLA-4 and anti-PD-1 checkpoint inhibitors, whose predicted single (or combined) actions in the context of peripheral B-cell tolerance, should be to enhance T helper cell activity, either in T-cell zones to promote early B-cell recruitment and expansion, or later during GC responses, to increase $\mathrm{T}_{\mathrm{FH}}$ activity. Similarly, appropriately administered anti-CD25 treatment should allow preferential depletion of Treg and $\mathrm{T}_{\mathrm{FReg}}$ populations (Belkaid and Tarbell, 2009). Thus, if the goal of a modified "bnAb-potentiating" vaccine strategy is alternatively (or additionally), to prolong vaccine-induced AM of bnAb lineages in GCs, for example during late boosts in sequential-based regimens (which will be detailed further 
below), then another type of modified "checkpoint-modulating" HIV regimen could be one transiently employing antibodies to CD25 in order to reduce $\mathrm{T}_{\text {Reg }}$ and $\mathrm{T}_{\mathrm{FReg}}$ numbers.

\subsubsection{Next generation of peripheral bnAb tolerance modulation: small molecule inhibitor-based regimens-While proving successful in cancer treatment,} the above strategies may nevertheless have various unintended, off-target effects, which in the setting of a preventative HIV-1 vaccine, would not pass the higher bar in terms of safety considerations. However, even were such approaches not be practical for clinical vaccine trials, they will still contribute to our emerging understanding of why even the best HIV immunogens and regimens available still fall well short of eliciting significant protective responses. Meanwhile, the search for more selective, ancillary small molecule inhibitor (SMI) strategies that can be administered locally (thus further reducing the likelihood of inducing off-target effects that systemic administration may evoke, should be aided by ongoing discovery of new signaling molecules/genes involved in $\mathrm{GC} / \mathrm{memory} / \mathrm{plasma}$ formation. Ultimately, to further specify and/or potentiate the precise delivery of such SMIs, administering them in subset targeted or more potent DNA/RNA platforms also under development by several groups could be used, and timing of their administration during vaccination will likely also likely be critical. Also noteworthy for all these endeavors is that they will require highly iterative testing, which is likely to best be done in appropriate bnAb KI models. As discussed in the previous section, distinct versions of such models are now emerging from several groups, which truly embody prototypes for different checkpoint blockades of distinct types of bnAb lineages, and many more will be reported shortly; thus an ample "arsenal" of bnAb models is anticipated to be available for such testing. Finally, several interesting candidates have been recently identified, having potentially key roles in the still poorly understood molecular programs of GC formation and selection involving the finer precise aspects of GC entry, duration, and exit as well as Tfh differentiation/activation, and thus for which developing novel, more selective small molecule inhibitors/activators and their subsequent iterative testing will be highly significant. Examples of such factors include molecules implicated in the GC program, such as IRF4 and IRF8, transcriptional regulators of the GC/plasmacyte axis master switches bcl6 and Blimp-1 (Klein et al., 2006; Lee et al., 2006; Sciammas et al., 2006), the AID-associated GC-restricted GTPase "SLIP-GC" (Richter et al., 2009; Richter et al., 2012), the GC-associated nuclear protein "GANP" (Kuwahara et al., 2004; Kuwahara et al., 2001; Sakaguchi et al., 2005; Singh et al., 2013), and other novel molecules involved in the Tfh differentiation program, like Activin (Locci et al., 2016).

\subsubsection{Small molecule inhibitor-based strategies for modulating central bnAb} tolerance-As with the small molecule inhibitor-based strategies noted above for releasing bn $\mathrm{Ab}^{+} \mathrm{B}$-cells from peripheral tolerance controls, those that precisely target genes regulating pathways involved in the central $\left(1^{\text {st }}\right)$ tolerance checkpoint could also be used, specifically in strains like 2F5 dKI mice, the prototypical central tolerance model (Fig 4), or in other newer KI models that will be emerging, with similar tolerization-associated developmental blockades in the bone marrow. Two current studies using small molecule inhibitors aimed at releasing bn $\mathrm{Ab}^{+} \mathrm{B}$-cells from central tolerance controls, in order to increase the frequency of bnAb precursors in the available naïve repertoire, are underway. In one study, promising 
results have just been reported in which transient use of the small molecule inhibitor hydroxyl-chloroquine, a compound already FDA-approved for malaria treatment, has been used to modulate a newly-identified axis involved in central tolerance, the BCR/Myd88/AID pathway (Kuraoka et al., 2017). In this study, the goal was to exploit the synergy between BCR and MyD88 signaling required for maintaining central B-cell tolerance and the knowledge that intracellular acidification is a necessary step in activating intracellular TLR signaling. Thus, to overcome Myd88/AID-mediated central tolerance, mature and UA 2F5 dKI mice were treated with a seven day regimen of hydroxy-chloroquine, an inhibitor of the key TLR acidification step, which markedly relaxed bnAb ${ }^{+} \mathrm{B}$-cell tolerance, as manifested by significantly increased numbers of transitional, mature follicular, and marginal zone $2 \mathrm{~F} 5$ $\mathrm{KI}^{+} \mathrm{B}$ cell subsets. In another study currently underway (L. Verkoczy in collaboration with B. Haynes, G. Kelsoe, personal communization), inhibitors of the PI3K signaling axis, another central tolerance/positive selection switch (wherein PI3K has been shown to be key for positively selecting immature bone marrow B-cells; (Llorian et al., 2007; Verkoczy et al., 2007)), are being evaluated. Specifically, various small molecule inhibitors selective for downstream negative effectors of PI3K (Benhamou et al., 2016; Coffre et al., 2016; Verkoczy et al., 2007), including those targeting foxo1, a transcription factor which plays a key role in promoting RAG expression required for LC receptor editing (and thus impeding positive selection) (Amin and Schlissel, 2008; Chow et al., 2013), are under investigation, for their abilities to increase $2 \mathrm{~F} 5 \mathrm{KI}^{+} \mathrm{B}$-cell frequencies and $\mathrm{BCR}$ signaling responsiveness. The recent identification of specific microRNAs regulating central tolerance signaling pathways (Coffre et al., 2016; Gonzalez-Martin et al., 2016; Lai et al., 2016), including the PI3K/foxo1 pathway (Coffre et al., 2016), presents yet another potential layer of transient manipulation that similar molecule-specific strategies could exploit in the near future.

\subsection{KI models for testing pathogenicity of bnAbs targeting self-mimicking Env epitopes}

As we noted above in section 4.1, Ab poly/autoreactivity, as defined by in vitro assays often, but not always correlate with in vivo tolerizing activity. This assertion is borne out by the presence of natural pools of B-cells that would be defined as polyreactive and/or autoreactive by standard clinical assays in all healthy individuals, including an estimated $20 \%$ of the mature naïve B-cell repertoire circulating in blood (Wardemann et al., 2003). Such "acceptable" in vitro poly/autoreactivity is also harbored by a large fraction within reservoirs of more specialized mature B-cells such as the peritoneal B-1 (Hardy, 2006) and splenic Marginal Zone (Martin and Kearney, 2002) compartments, the latter in particular also shown to be enriched for specificities resembling precursors of some bnAbs such as the VRC01 class (Pujanauski et al., 2013). Furthermore, there also exist examples of mAbs clinically identified as auto-Abs, that have been subsequently shown to have no effect on Bcell ontogeny when transgenically expressed in mice as BCR specific for the Pemphigus autoantigen, DSG3 (Ota et al., 2004) or the La antigen of SLE (Aplin et al., 2003). Conversely, and more likely the case (due to current technical and theoretical limitations with relevant detection methodologies) Abs with tolerizing specificities could be missed in screens using standard autoantigen panels, including ones exhibiting tissue specific expression, or with ultra-low in vitro affinities to effect tolerance controls in vivo. Examples of such Abs include those knocked-in or transgenically introduced as BCRs specific for physiological antigens like erythrocytes (Murakami et al., 1992), pancreatic insulin B-islets 
(Rojas, Hulbert and Thomas, 2001), IgG2 (Wang and Shlomchik, 1997), ATA determinant, i.e. glycosylated Thy-1 (Hayakawa et al., 2003), and Goodpasture antigen a 3 collagen (Zhang et al., 2008).

A related concept to the one above, namely that in vitro poly/autoreactvity of Abs does not always evoke in vivo tolerization, is that self-reactive Abs capable of evoking B-cell deletion/anergy do not necessarily trigger pathogenic effects, even after have undergone IgG-switching and/or extensive SHM. However, since accumulating data in multiple bnAb $\mathrm{KI}$ model studies predicts many (if not most) bNAb lineages (even those lacking detectable in vitro poly-autoreactivity, e.g. 3BNC60) will most likely be limited by various B-cell tolerance controls, at some point in their evolution, it will be critical to know which lineages capable of generating breadth pathogenic, in order for their safe elicitation by vaccine strategies. In this regard, bnAb KI mice, which have already proven to be especially powerful platforms to dissect the fate of bnAb lineages in various immune tissues and to test if and how to re-activate tolerized bnAb lineage members, can now also be used, along with more powerful in vitro screening methodologies to evaluate their polyreactivity versus autoreactivity, such as by proto-array (Bonsignori et al., 2014; Liao et al., 2013b; Liu et al., 2015; Yang et al., 2013) and PhIP-Seq library analysis (Finton et al., 2013; Larman et al., 2011), to better understand what types of re-activated self-reactive bnAbs may be more problematic, in terms of any potential adverse pathogenic effects. Ultimately, these types of platforms can also be used to directly test for any negative, off target physiological effects of any promising bnAb lineage that has been already studies in such models.

An example of the utility of bnAb KI models for the purposes noted above, is a series of studies done in the related, original (affinity matured) 2F5 and 4E10 dKI models. Specifically, two earlier studies have found that 2F5 and 4E10 exhibit vastly different in vitro self-reactivity profiles, despite their proximal epitopes in gp141 MPER. In an earlier study, 4E10 was found to exhibit substantially higher reactivity to lipids than does 2F5 (Haynes et al., 2005a). In a later study, using more comprehensive screening of 9500 host proteins using protein arrays (Yang et al., 2013), 4E10 was found to not only have strong affinity for an RNA splicing factor, splicing factor 3b subunit 3 (SF3B3), but also exhibited exceptionally broad polyreactivity, while $2 \mathrm{~F} 5$ demonstrated no/minimal polyreactivity, but instead, had high, specific affinity for Kynurinenase, a highly-conserved candidate autoantigen involved in trypophane metabolism, that represents the only known protein to contains an epitope in its a-helical $\mathrm{H} 4$ domain that is an exact sequence mimic of the linear 2F5 minimal epitope specified by the ELDKWA motif found in the gp41 MPER ectodomain. Notably, the specific in vivo tolerization patterns observed in the 2F5 and 4E10 dKI models also appeared to mirror the differential in vitro reactivities identified for these two bnAbs (Chen et al., 2013b). In particular, LC editing selectively mediated removal of the 2F5 MPER epitope in peripheral follicular B-cells of mature 2F5 dKI mice, whereas it preferentially targeted polyspecific/lipid binding (over MPER epitope binding) in those from 4E10 mature dKI mice. Also of note, induction of serum Abs to the 4E10 epitope in MPER has proved more difficult than inducting those directed to the 2F5 epitope, upon immunization with similar MPER- liposome-based regimens, in several experimental animal models, including opossums, 2F5/4E10 dKI mice, and in Rhesus macaques ((Yang et al., 2013; Zhang et al., 2016) and H. Bouton-Verville, L. Verkoczy et al., manuscript in 
preparation), further suggesting disparate in vivo outcomes of vaccination in inducing nAbs directed to these related epitopes.

Of highest relevance however, is that in addition to the consequences of the distinct in vitro self-reactivities these related $\mathrm{MPER}^{+}$bnAbs effect on tolerization and immunization, they have now also been found to distinctly impact pathogenicity. In particular, while significant lupus anti-coagulant activity and blocking effects in clotting tests has been reported for 4E10 in vitro (likely at least partly resulting from its strong reactivity to cardiolipin), none was reported for 2F5 (Li et al., 2011). Furthermore, when passively administered to humans, 4E10 was biologically active and exhibited prolonged partial thromboplastin and prothrombin times in vivo, whereas no obvious clinically adverse effects were reported in passive infusions with 2F5 (Vcelar et al., 2007). Thus, while 4E10 has clearly been demonstrated to have negative physiologic effects both in vitro and in vivo associated with its broad polyspecificity and affinity for lipids, 2F5 did not appear to have any such effects in similar assays. However, any other off-target effects resulting from its induction could not be ruled out in these earlier studies, given that its specificity for Kynureninase had not yet been determined, nor had it's the physiological significance of its autoreactivity been examined in a vaccination setting. Remaining concerns about eliciting 2F5-like bnAbs as a safe vaccine target have largely been alleviated in a recently completed pathogenicity study performed in both the 2F5 mature dKI model and in Rhesus macaques immunized with the MPER-liposome regimen (Bradley et al., 2016). In this report, despite elicitation of high circulating plasma IgG $2 \mathrm{~F} 5$ in $2 \mathrm{~F} 5 \mathrm{dKI}$ mice or 2F5-like Kynureninase cross-reacting $\mathrm{Ab}$ titers in the non-human primates, no associated autoimmune disease were seen in either model. Furthermore, no disruptions in Kynurinenase enzyme activity or tissue tryptophan metabolism were observed in blood or in numerous tissues assessed, including the brain, indicating the $2 \mathrm{~F} 5 \mathrm{bnAb}$ 's inherent autoreactivity does not induce tissue damage in vivo.

As has been previously noted (Haynes et al., 2005b; Kelsoe, Verkoczy and Haynes, 2014), host mimicry by HIV-1, for example, that involving 2F5's linear MPER epitope with Kynureninase (Yang et al., 2013), provides a powerful method of concealing its vulnerable bnAb epitopes. Such epitopes essentially "hide in plain sight", via the various selection processes that purge the very B cells required for protective immunity, i.e. those acquiring bnAb specificities. Thus, in addition to their clinical relevance in confirming nonpathogenicity of bnAbs reactive for self-mimicking Env epitopes (e.g. 2F5), studies in mature versions of bnAb KI models (e.g. the "original" 2F5 dKI strain noted above), in combination with host protein arrays for defining physiologically-distinct outcomes of 4E10 and 2F5 self-reactivities, also raise some fundamental questions for HIV vaccinology, regarding both prevalence of HIV host mimicry at different bnAb epitope clusters in Env, and the potential relationship of any such mimicry with pathogenicity.

First, regarding the prevalence of HIV mimicry, other potential bnAbs like 2F5, with no/ minimal polyspecificity but conserved autoreactivity such as many of the CD4bs ${ }^{+}$bnAbs have with UBE3A ligase (Liu et al., 2015), and thus may represent only the tip of the iceberg. Indeed, antigenic mimicry of mammalian host structures has been described as an effective evasion mechanism for many bacterial and fungal pathogens (reviewed in (Patel and Kearney, 2016)), including some bacterial examples such as neuronal ganglioside 
mimicry by bacterial liposoligosaccharides (LOS) from Campylobacter jejuni strains (Bowes et al., 2002; Yu et al., 2011), Lewis blood antigen group mimicry by LOS of Helicobacter pylori strains (Appelmelk et al., 1996), and mimicry by the adhesin FimHI (expressed on fimbriated bacteria) of lysosomal membrane protein-2 (LAMP-2) (Kain et al., 2008). Furthermore, many of these examples involve bacterial mimicry of glycan-dependent epitopes, which thus implies HIV mimicry may not just be relevant to MPER and CD4bs ${ }^{+}$ bnAbs, but given the unusual degree of glycosylation that Env uses to cloak many of its vulnerable sites (Burton and Hangartner, 2016; Pantophlet and Burton, 2006), this likely extends to the V2 and V3 glycan-dependent bnAb epitope clusters in Env. One potential vaccine strategy based on exploiting glycan mimicry to break tolerance of cross-reactive bnAb epitopes in such potential regions has been one identifying the striking similarities of rhizobial LOS backbone with mammalian oligomannose (Clark et al., 2012), which was demonstrated structurally by a crytal structure of the glycan-dependent bnAb 2G12 with this bacterial structure (Stanfield et al., 2015). One potential logistic issue with vaccine strategies that exploit HIV mimicry however, is the previously-discussed concept of affinity reversion/Ab redemption (Haynes, Verkoczy and Kelsoe, 2014; Sabouri et al., 2014a; Verkoczy et al., 2013; Verkoczy and Diaz, 2014), since the cross-reactivity of Ab specificities generated by AM in the GC reaction are likely to be close between the revenant self and foreign antigens, and thus could result in especially protracted rounds of "conflicted selection". Thus, appropriate boosting for such approaches will not only require highlydetailed knowledge of what these precise overlaps between self and non-self antigens are, but also highly-iterative in vivo platform like bnAb KI models for testing immunogens designed based on such considerations.

Secondly, regarding the issue of HIV mimicry and potential pathogenicity, it is tempting to speculate that other bnAbs, similar to $2 \mathrm{~F} 5$, with more directed, HIV mimicry-associated specificity (and as opposed to bnAbs with more general "off-target" polyreactivities like 4E10), may have higher likelihoods of also lacking pathogenicity. However, not all hostresembling epitope expression by pathogens may be devoid of inducing "off-target" physiology effects, as in some cases, such microorganisms have been implicated (albeit somewhat loosely) in autoimmunity development (Cusick, Libbey and Fujinami, 2012). Nevertheless, this again points to the importance of testing pathogenicity on a "case-bycase" basis, for any bnAbs lineage for which a viable vaccine strategy based on HIV mimicry can be developed, using relevant bnAb KI models expressing such specificities. Since many more such models are now being made due to emerging high-throughput technology (that will be discussed further below in Section 6), this additional, relevant layer of testing should become rather routine.

\section{5. bnAb KI models for testing new HIV vaccine strategies}

\subsection{The advent of bnAb precursor-targeting immunogens}

A potentially key roadblock which has been identified for HIV vaccine design, and for inducing bnAbs in particular, has been the observation that most recombinant Env immunogens the field has been generating and testing up until relatively recently, interact sub-optimally with unmutated ancestor or experimentally-reverted "precursor" versions of 
original (affinity-matured) bnAbs (Haynes et al., 2012b; Hoot et al., 2013; Mascola and Haynes, 2013; Ota et al., 2012; Xiao et al., 2009). This has led to the hypothesis that most naïve B-cells in the pre-immune repertoire, which express unmutated precursor bnAbs as BCRs, fail to be activated by such immunogens, because they either fail to engage them altogether, or bind them at affinities that in theory, would be insufficient for priming them in vivo (Dal Porto et al., 2002). A related hypothesis is that Env immunogens in non-native forms not only bind bnAbs at insufficient affinities, but also lack adequate specificity for them, instead binding multiple other "off-target" paratopes on competing naïve B-cell clones (Mascola and Haynes, 2013; McGuire et al., 2014; Pantophlet and Burton, 2006; Wu et al., 2011). Thus, to overcome these limitations, considerable efforts by several groups in the last few years have been placed on designing immunogens that can specifically target and engage precursors of various bnAb classes (Alam et al., 2013a; Alam et al., 2013b; Andrabi et al., 2015; Bhiman et al., 2015; de Taeye et al., 2015; Gorman et al., 2016; Hoot et al., 2013; Jardine et al., 2013; Kwon et al., 2015; Liao et al., 2013a; Liao et al., 2013b; Ma et al., 2011; McGuire et al., 2013; Sanders et al., 2015; Sliepen et al., 2015).

Several types of approaches have been employed in designing such "bnAb priming" immunogens. One of the two original strategies has been to use time-of-infection information from longitudinal sampling (Bhiman et al., 2015; Liao et al., 2013b), in particular, Env of the transmitted/founder (TF) which binds the bona fide unmutated common ancestor (UCA) of a given HIV1 infected subject's co-evolved bnAb lineage, as the template from which the priming immunogen is generated. While this concept of deriving "natural" Env priming immunogens from a given bnAb co-evolved lineage's TF virus was first described for the "HCDR3-binder" CD4bs+ bnAb lineage CH103 (Liao et al., 2013b), similarly-derived priming immunogens for other lineages have now also been described, including the $\mathrm{CH} 103$-co-operating "CD4 mimic" CD4bs+ bnAb lineage $\mathrm{CH} 235$ (Bonsignori et al., 2016; Gao et al., 2014) and the V1V2/glycan-directed bnAb lineage VRC26-CAP256 (Bhiman et al., 2015; Doria-Rose et al., 2014). The alternative, original strategy proposed for developing bnAb precursor-priming immunogens is a "reverse engineering" approach, which involves a combination of in vitro mutation/selection and in silico computational methodologies to synthetically engineer candidate immunogens capable of binding multiple precursors and mature BCRs within a given bnAb "class" i.e. directed to a common bnAb epitope cluster in Env (Jardine et al., 2013; McGuire et al., 2013). Thus far, such "germlinetargeting" (GT) immunogens have been experimentally-generated for the "VRC01/CD4 mimic" class of CD4bs+ bnAbs, based on this reverse engineering approach, and thus far, amongst all the candidate bnAb precursor-directed immunogens, have also been the most extensively evaluated, in terms of their ability to prime and B-cells in vivo, using several VRC01 germline KI models, which will be discussed in detail in the next section. Finally, three additional, novel approaches have been described to enhance affinities at which bnAb precursors can bind near-native trimeric Env immunogens. Such near-native trimers are already thought to be more specific for bnAbs than other forms of Env immunogens (such as monomeric gp120s), due to being in more "closed" conformations that should allow greater access to bnAb epitopes, while occluding greater numbers of non-nAb epitopes, the former because certain bnAb epitopes are only being present when Env is in native-like conformations and the latter because appropriate constraints are placed on the angles at 
which Abs can approach various key Env regions of vulnerability (de Taeye, Moore and Sanders, 2016; de Taeye et al., 2015; Sanders et al., 2015; Sliepen and Sanders, 2016). Two such "enhancement" strategies for generating higher affinity versions of near-native trimers have been employed on those engineered to preferentially target V2/glycan+ bnAb precursors. One of these strategies has been to select such immunogens from large-scale screens of viral isolates that can neutralize "germline-reverted" versions of several prototypical V2/glycan+ bnAbs e.g. PG9, CH01, PGT145, CAP256 (Andrabi et al., 2015). Alternatively, higher-affinity, V2/glycan-targeting near-native trimers have been produced by engineering chimeric trimers, in which $\mathrm{V} 2$ /glycan epitopes from viral isolates having enhanced binding to V2/glycan+ bnAb UCA precursors have been grafted into "DS-Env" stabilized trimers (Gorman et al., 2016; Kwon et al., 2015). The third recent, potentially most comprehensive approach currently underway for making such enhanced trimeric immunogens, is a series of additional modifications being made in the best described a native-like trimer, the BG505 SOSIP 664 Env platform, which has been stabilized by disulfide bonds to link gp120 and gp41 (which has been truncated at position 664 resulting in deletion of the MPER), as well as an additional mutation to stabilize gp41. This stabilized, near-native trimer immunogen can already bind certain classes of bnAb precursors with appreciable affinity e.g. V1V2/glycan-directed bnAb precursors (Sliepen et al., 2015); but additional mutations are currently being designed to further enhance binding to various CD4bs+ and/or V1V2/glycan+ bnAb UCA/reverted precursors (R. Sanders, personal communication).

All these above-noted priming immunogens have thus not only been selected for their specificity in targeting bnAb precursors, but their ability to bind them at low uM (and often, $\mathrm{nM}$ ) affinities, based on the rationale that these affinities are well above minimal theoretical thresholds for effectively engaging and activating the pre-immune B-cell repertoire in vivo (Dal Porto et al., 2002; Dal Porto et al., 1998; Shih et al., 2002) and thus have been generated with the intent of "kick-starting" such bnAb precursors and/or initiating lineage development in vivo. In some cases, the potential of such germline-targeting immunogens for priming/activating bnAb precursors has been further validated by demonstrating their capability to induce robust calcium flux in bnAb-transfected B-cells in vitro (Jardine et al., 2013; McGuire et al., 2013). In the next section, we will review some of the initial immunization priming studies done and/or underway in various KI models for evaluating candidate bnAb precursor-priming immunogens, some (mostly for the VRC01 class bnAbs) which are now beginning to demonstrate they indeed can activate and elicit bnAb precursors, and thus behave in vivo as have been hoped.

\subsection{Evaluation of bnAb activation/priming by precursor-targeting immunogens in $\mathrm{KI}$ models}

Immunization studies in various types of Ig humanized KI models have now begun to provide convincing evidence, at least for certain bnAb classes, that "precursors" of bnAbs can be kick-started by "priming" immunogens. As noted above, much of this work is still very much at the proof-of-concept stage, since it has focused thus far predominantly on evaluating immunogens for activating precursors of one bnAb type in particular, the VRC01like ("CD4 mimic") class of CD4bs ${ }^{+}$bnAbs, using several KI models, all engineered to 
express human $\mathrm{V}_{\mathrm{H}} 1-2$, a germline segment common to these bnAbs. Below however, we will not only discuss these studies in detail, but also review other, more recent "priming studies" to evaluate immunogens targeting precursors of the V3 high-mannose PGT121-like ("N332 supersite") class of bnAbs, as well as currently- ongoing investigations of immunogens aimed at initiating other bnAb classes/lineages, in various novel KI models.

To evaluate priming immunogens targeting precursors of the "CD4 mimic" class of CD4bs ${ }^{+}$ bnAbs, two precursor bnAb KI models, VRC01gH and $3 \mathrm{CBN} 60 \mathrm{GLV}_{\mathrm{H}}$ were constructed (Dosenovic et al., 2015; Jardine et al., 2016a). These models were engineered to forced expression of $\mathrm{V}_{\mathrm{H}} \mathrm{DJ}_{\mathrm{H}}$ rearrangements (via their integration into the $\mathrm{J}_{\mathrm{H}}$ cluster) of either VRC01 or 3CBN60, a VRC01 family member. Both rearrangements used the common germline $\mathrm{V}_{\mathrm{H}} 1-2$ segment, but retained the mutated HCDR3 regions of the original VRC01 and 3CBN60 bnAbs, respectively (Figs 6A,B). In both models, B-cell development appeared to be largely normal. Furthermore, in the VRC $01 \mathrm{gH}$ model, $85 \%$ of B-cells expressed the VRC01gH (Jardine et al., 2015), whereas essentially all naive B-cells in the $3 C_{B N 60}$ GLV $_{H}$ model expressed the "knocked-in" $\mathrm{GLV}_{\mathrm{H}}-3 \mathrm{BNC60}$ rearrangement (Dosenovic et al., 2015). In both cases, the cognate bnAb LCs were not knocked in, under the premise that mouse LCs with 5-aa CDR L3 could function in association with $\mathrm{V}_{\mathrm{H}} 1$-2-expressing HCs as VRC01 precursors.

With respect to the VRC01gH model specifically, immunization studies were performed to evaluate the efficacy of a candidate VCR01 Ab-priming immunogen, eOD-GT8 (Jardine et al., 2015). This immunogen was derived from the original concept of converting Env into a form that could bind VRC01 precursor Abs with sufficient affinity, by overcoming the major impediment to this: removing the CD4bs-proximal N-linked glycans near the CD4bs. Removal of these glycans was done by mutating the glycosylation site at N276 in loop D and the N460 and N463 sites in the V5 domain of a clade C virus (426c), significantly enhanced the binding affinity between the mutant Env and precursor VRC01 Abs (McGuire et al., 2013). However, the triple glycosylation mutations alone, did not provide a complete solution to the problem of Env/glVRC01 interactions, since the triple mutant 426c Env bound some, but not all, precursor VRC01-class Abs. To deal with this issue, a more dramatic modification of Env, i.e. engineering a minimal gp120 outer domain (eOD), was the starting point for development of the eOD-GT8 immunogen (Jardine et al., 2013; Jardine et al., 2016a). While the original eOD base interacted poorly with gl-VRC01 Abs, it was eventually engineered into the broad precursor VRC01-binder, eOD-GT8, via several iterations of structure-based modifications (Jardine et al., 2016a).

For immunization purposes, eOD-GT8 was formulated as 60-mer nanoparticles, with the goal of binding BCRs at higher avidity than as a monomer, thus potentially activating B-cell cross-linking. Immunization of VRC01gH mice with eOD-GT8 60mer elicited memory Bcells that expressed VRC01-like Abs. Specifically, about 90\% of CD4bs-specific IgG ${ }^{+}$Bcells expressed Abs bearing the VRC01gH paired to mouse LCs with 5-aa CDRL3s; moreover, the CDRL3s were enriched for a partial VRC01 consensus motif QQYXX. Interestingly, amongst the adjuvants used to formulate the eOD-GT8 60-mers with (alum, Iscomatrix, and Ribi), Ribi appeared to induce the most SHM. Functionally, these Abs bound specifically to the CD4bs, but exhibited no neutralization activity, owing to the 
limited overall SHM frequencies observed. Nevertheless, these results indicate that the eODGT8 60mer is an effective priming antigen for activating B-cells expressing VRC01 precursor Abs. In contrast, immunization with the native trimeric Env protein, BG505 SOSIP, elicited no VRC01-like Abs, likely due to its poor interaction with VRC01 precursors.

Similar immunization "priming-phase" studies have also been done in the germline-reverted "3BNC60 $\mathrm{GLV}_{\mathrm{H}}$ model", to evaluate the ability of eOD-GT8, and a second candidate VCR01 germline-targeting immunogen, multimeric 426c TM4 $\Delta \mathrm{V} 1-3$, to prime VRC01 class bnAb precursors (Dosenovic et al., 2015). The 426c.TM4 $\Delta$ V1-3 immunogen (McGuire et al., 2016) contains mutations in three $\mathrm{N}$-linked glycosylation sites near the CD4bs as well as truncations of variable loops 1-3 ( $\Delta \mathrm{V} 1-3)$, modifications that significantly improved the binding affinity of 426c Env toward VRC01 Ab precursors. One or two immunizations of 3BNC60 $\mathrm{GLV}_{\mathrm{H}}$ mice with these germline-targeting immunogens induced robust $\mathrm{Ab}$ responses; and some had CD4bs-specificity, especially so with eOD-GT8. As seen in the VRC01 gH model, B-cells expressing 5-aa CDRL3s were highly infrequent in the naïve repertoire of 3BNC360 $\mathrm{GLV}_{\mathrm{H}}$ mice, but CD4bs-specific sorted memory cells with 5-aa CDRL3 signatures could be found after priming. Also like the VRC01 gH model, multiple immunizations with recombinant native-like trimers Envs failed to elicit significant CD4bsspecific serum Ab responses, as would be expected, since the reverted 3BNCC60 Ab fails to bind this trimer in vitro. Additionally, neither mAbs nor serum Abs in mice from which 5-aa CDRL3s were observed exhibited neutralization activity, also as expected from priming with a single precursor-targeting immunogen (and no boosting). However, one notable difference in this model is that only one out of four immunized mice displayed the classical 5-aa CDRL3 signature, and only a minor fraction of clones sorted for CD4bs-specificity exhibited this feature, in contrast to the majority sorted from immunized VRC01 $\mathrm{gH}$ mice.

Interestingly, when parallel immunization studies were carried out in a version of the 3BNC60 KI mouse model $\left(\mathrm{MuV}_{\mathrm{H}}\right)$ that instead of expressing germline-reverted $\mathrm{V}_{\mathrm{H}} \mathrm{DJ}_{\mathrm{H}}$ rearrangements, expressed mature $3 \mathrm{BNC} 60 \mathrm{~V}_{\mathrm{H}} \mathrm{DJ}_{\mathrm{H}}$ rearrangements (Dosenovic et al., 2015) (Fig 5C). In this system, an Ab composed of mature 3BNC60 HC pairing with a $\mathrm{LC}$ with 5aa CDR L3 was thought of as a synthetic 3BNC60 "intermediate", and the model was designed to test the ability of immunogens to promote further maturation of this intermediate, primarily through optimization of the LC. The introduction of a mature 3BNC60 HC into this model dramatically improved its immune response to eOD-GT8 60mer and native Env trimer, BG505 SOSIP. In both cases, immunization led to marked induction of CD4bs-specific Abs and neutralization activities in sera. Correspondingly, the frequency of CD4bs-specific $\mathrm{IgG}^{+} \mathrm{B}$-cells in $\mathrm{MuV}_{\mathrm{H}}$ mice increased dramatically in response to immunization with either eOD-GT8 or BG505 SOSIP, and most of these B-cells expressed mouse LCs with 5-aa CDR L3s, which contains an E residue at the $4^{\text {th }}$ position, a signature of VRC01-class Abs. Moreover, these cloned Abs were CD4bs-specific and recapitulated the serum neutralization activities. Overall, BG505 SOSIP elicited broader neutralization activities than eOD-GT8 60mer. These results reinforce the notion that maturation of intermediate Abs will require more native-like Env immunogens, an issue that will be discussed in detail in below sections regarding boosting concepts for broadening bnAb responses. 
The reason for this dramatically lower induction of VRC01-like Abs in the 3BNC60 GLV $\mathrm{B}_{\mathrm{H}}$ model, relative to the VRC01gH model, is intriguing, and remains to be formally determined, but can likely be attributed to differences in binding affinities that VRC01 $\mathrm{gH}$ and 3BNC60 $\mathrm{GLV}_{\mathrm{H}}$-expressing B-cell clones have for eOD-GT8 in vivo. As already noted, both knocked-in $\mathrm{HCs}$ utilize the same germline $\mathrm{V}_{\mathrm{H}} 1-2 * 02$ segment, but contain HCDR3 from the mature VRC01 and 3BNC60 bnAbs. In this regard, when the reverted/gl-VRC01 and 3BNC560 are made in vitro as IgG Abs, eOD-GT8 interacts with gl-VRC01 Ab at roughly a log higher affinity than does gl-3BNC60. Alternatively (or additionally), while the overall pre-immune B-cell repertoire in the $3 \mathrm{BNC} 60 \mathrm{GLV}_{\mathrm{H}} \mathrm{KI}$ model is not under any obvious developmental blockades, the rare clones expressing 5-aa CDRL3s may be under negative selection mechanisms, thus may be at a selection/survival disadvantage, relative to off-target clones (which are not under tolerance control), in response to immunization. Consistent with this possibility, is the finding that unlike the normal pre-immune B-cell repertoire of 3BNC60 $\mathrm{GLV}_{\mathrm{H}}$ mice (which only express the gl-reverted $\mathrm{HC}$ rearrangement, and can thus pair with diverse mouse LCs), that of 3BNC60 $\mathrm{GLV}_{\mathrm{HL}}$ mice, a model forced to initially express not only the gl-reverted HC rearrangement, but also the 5aa-HCDR3containing gl reverted-LC rearrangement of 3BNC60, is under considerable negative selection (McGuire et al., 2016), including exhibiting partial clonal deletion in the bone marrow, and a residual peripheral repertoire that is largely comprised of naïve B-cells which have undergone receptor editing via replacement of their knocked-in germline-reverted 5-aa LCDR3-containing LCs with endogenous mouse $\lambda \mathrm{LCs}$, and clones which did manage to retain both their $\mathrm{KI}$ gl HC+LCs were under anergy controls (Fig 5D).

A significant caveat with the priming studies done in the above-described VRC01 $\mathrm{gH}$ and $3 \mathrm{BNC60} \mathrm{GLV}_{\mathrm{H}}$ models is that in retaining mature HCDR3s from the original bnAbs, they are not equivalent to bona fide VRC01 precursors. Furthermore, both models express prerearranged VDJs bearing $\mathrm{V}_{\mathrm{H}} 1-2$ linked to fixed HCDR3s, whereas in the human $\mathrm{Ab}$ repertoire, $\mathrm{V}_{\mathrm{H}} 1-2$ is linked to diverse HCDR3s, a subset of which may be compatible with the development of VRC01-like Abs. Thus, to evaluate the validity of eOD-GT8 as a priming immunogen in a repertoire with diverse VRC01 precursors (i.e. in a setting where human $\mathrm{V}_{\mathrm{H}} 1-2$ could be expressed in association with a repertoire of HCDR3s), a " $\mathrm{V}_{\mathrm{H}} 1-2$ " $\mathrm{KI}$ model has more recently been generated, where the human $\mathrm{V}_{\mathrm{H}} 1-2$ segment is knocked-in to selectively replace a single mouse $V_{H}$ segment (Fig 5E) (Tian et al., 2016). Since unrearranged $\mathrm{V}_{\mathrm{H}} 1-2$, along with mouse $\mathrm{D}$ and $\mathrm{J}_{\mathrm{H}}$ segments needs to undergo normal $\mathrm{V}(\mathrm{D}) \mathrm{J}$ recombination in this system, the frequency of B-cells expressing potential VRC01 precursors are expected to be very low, thus presenting practical difficulties for immunization studies. In order to circumvent this issue, i.e. generate a diverse repertoire of $\mathrm{V}_{\mathrm{H}}$ 1-2-expressing $\mathrm{Abs}$, but at high enough frequencies to allow for a robust immunization readout, the $\mathrm{V}_{\mathrm{H}} 1-2 \mathrm{KI}$ model was engineered with two specific modifications. First, $\mathrm{V}_{\mathrm{H}} 1-2 * 02$ was substituted specifically for mouse $\mathrm{V}_{\mathrm{H}} 81 \mathrm{X}$, the $\mathrm{V}_{\mathrm{H}}$ segment at the endogenous $\mathrm{IgH}$ locus that is most proximal to the $\mathrm{D}$ segments, which at least in part explains its most frequent-utilization amongst all $\mathrm{V}_{\mathrm{H}}$ segments, during $\mathrm{HC}$ rearrangement (Alt et al., 2013). Secondly, the bias for $\mathrm{V}_{\mathrm{H}} 81 \mathrm{X}$ rearrangement was made even more pronounced, by deleting a regulatory element at the intergenic region between $\mathrm{V}_{\mathrm{H}}$ and $\mathrm{D}$ segments, IGCRI (Guo et al., 2011). Thus, by replacing $\mathrm{V}_{\mathrm{H}} 81 \mathrm{X}$ with $\mathrm{V}_{\mathrm{H}} 1-2 * 02$, in the 
context of IGCRI deletion, approximately $45 \%$ of peripheral B-cells expressed $\mathrm{V}_{\mathrm{H}} 1-2$ in this KI model (Tian et al., 2016), yet could still retain their ability to recombine with all mouse D and $\mathrm{J}_{\mathrm{H}}$ segments, and thus their potential for creating a diverse set of HCDR3s. Given these features, this model provided the opportunity to evaluate the ability of eOD-GT8 to selectively engage the VRC01 precursor amongst a diverse pool of $\mathrm{V}_{\mathrm{H}} 1-2 * 02+\mathrm{B}$-cells. Priming of this model with the eOD-GT8 60mer formulated with poly I:C adjuvant elicited robust $\mathrm{IgG}^{+} \mathrm{CD}_{4 \mathrm{bs}^{+}}$serum $\mathrm{Ab}$ and memory B-cell responses. Of the LCs expressed by $\mathrm{CD} 4 \mathrm{bs}^{+}$memory B-cells, the frequency of 5-aa CDRL3s, although minor (in the same range as those elicited in the 3BNC60 model) was considerably higher than those found in the preimmune repertoire, and higher doses $(60 \mu \mathrm{g})$ of eOD-GT8 appearing elicited the highest frequencies. Along with the 5-aa CDRL3 signature, CDRL3s were enriched for the partial consensus VRC01 motif of QQYXX, also as found previously in the standard VRC01 $\mathrm{gH}$ and $3 \mathrm{BNC60} \mathrm{GLV}_{\mathrm{H}} \mathrm{KI}$ models. In binding assays, the elicited VRC01-like Abs were specific for CD4bs, but also like in the other models, had not matured sufficiently to develop neutralization activity. Thus, these results indicate that even in a more challenging environment, i.e. that expressing a more complex repertoire capable of generating diverse HCDR3s, it was still feasible to selectively activate B-cells expressing VRC01-like precursors with the high-affinity VRC01-class priming immunogen, eOD-GT8.

Although the $\mathrm{V}_{\mathrm{H}} 1-2 \mathrm{KI}$ model contains a diverse repertoire of $\mathrm{V}_{\mathrm{H}} 1-2 * 02^{+} \mathrm{HCs}$, the frequency of $\mathrm{B}$ cells expressing $\mathrm{V}_{\mathrm{H}} 1-2 * 02(45 \%)$ was far higher than in the human $\mathrm{Ab}$ repertoire ( 3\%) (Arnaout et al., 2011; DeKosky et al., 2015; Sok et al., 2016). Furthermore, this model uses mouse $\mathrm{D}$ and $\mathrm{J}$ regions, the latter that lack high homology to human $\mathrm{J}$ segments. Thus, as the "highest bar" to evaluate the potential of eOD-GT8 to engage genuine VRC01 precursors, two related studies have been more recently reported. First, the frequency of such bona fide precursors in humans was evaluated, by isolating eOD-GT8 binding B-cells from human peripheral blood (Jardine et al., 2016a). From 61.6 million naïve B-cells, 26 isolated B-cells (1 in 2.4 million) were found to express Abs bearing $\mathrm{V}_{\mathrm{H}} 1-2 \mathrm{HC}$ and LCs with 5-aa CDRL3s. Moreover, based on structural studies, isolated VRC01-like Abs bound the CD4bs essentially the same way as VRC01-class Abs. Secondly, to further evaluate the efficacies of eOD-GT8 as a priming immunogen in a fully polyclonal and Ig-humanized environment, priming with eOD-GT8 was conducted in Kymab mice, in which the complete human $\operatorname{IgH}$, Ig $\kappa$ and $\operatorname{Ig} \lambda$ minigene segment repertoires were incorporated into the corresponding mouse loci (Lee et al., 2014). In these mice, human $\mathrm{V}_{\mathrm{H}} 1-2 \mathrm{HC}+\mathrm{B}-\mathrm{cells}$ represent somewhat similar frequencies $(\sim 1 \%)$ as found in the human repertoire (Arnaout et al., 2011; DeKosky et al., 2015; Sok et al., 2016), but those with 5-aa CDRL3s are found at $0.018 \%, 90$ fold lower than in humans (Sok et al., 2016). When accounting for the lower total B-cell numbers mice have, relative to humans, VRC01-like precursors were expected to be even more infrequent in Kymab mice than in humans. Supporting this prediction, no VRC01-class B-cells were identified by eOD-GT8 sorting of 300 million B cells from naïve Kymab mice (Sok et al., 2016); using the same approach for detecting the 1 in 2.4 million in humans (Jardine et al., 2016a). In spite of this paucity of VRC01-class precursors in Kymab mice, priming with the eOD-GT8 60mer elicited VRC01-like Abs in $29 \%$ of immunized animals, with $1 \%$ of CD4bs ${ }^{+}$Abs in responding mice expressing $\mathrm{V}_{\mathrm{H}} 1-2 \mathrm{HCs}$ paired to various $\mathrm{LCs}$ that corresponding to those found in 
VRC01-class Abs, and which bore 5-aa CDRL3s and (Sok et al., 2016). As with the VRC01 $\mathrm{gH}, 3 \mathrm{CBN60} \mathrm{GLV}_{\mathrm{H}}$, and $\mathrm{V}_{\mathrm{H}} 1-2 \mathrm{KI}$ models, these CDRL3s were also enriched for the partial VRC01 consensus QQYXX. Also similar to the priming results in the other KI models, none of the elicited VRC01-like Abs matured enough to attain neutralization activities, and immunization with a near native Env trimer, BG505 SOSIP, failed to elicit VRC01-like Abs in the Kymab model. Thus, these studies represent the most stringent test proving that eODGT8 can serve as an effective priming immunogen, and along with studies evaluating the multimeric 426 core immunogen, provides a beachhead that priming with immunogens capable of binding VRC01 precursors at high affinity in vitro are required to activate/initiate development of VRC01-class Abs in vivo.

Also very recently, evaluation of other priming immunogens, those aimed at inducing the V3 high mannose patch-specific "N332 supersite" class of bnAbs, have been conducted in two PGT121 KI models (Escolano et al., 2016). One of these models, $\mathrm{GL}_{\mathrm{HL}} 121$, was constructed to express a "precursor" germline (gl) PGT121 Ab (Steichen et al., 2106), whose HC rearrangement is comprised of germline $\mathrm{V}$ and $\mathrm{J}$ segments plus the HCDR3 of the least mutated member in the PGT121 lineage, while the other model, Mut ${ }_{\mathrm{H}} \mathrm{GL}_{\mathrm{L}} 121$, was constructed to express a synthetic intermediate PGT121 Ab, comprised of the original (affinity-matured) PGT 121 bnAb's mutated HC rearrangements, but paired to the glPGT121 LC rearrangement. As with the VRC01gH and 3CBN60 $\mathrm{GLV}_{\mathrm{H}}$ models noted above, the rearranged $\mathrm{V}_{\mathrm{H}} \mathrm{DJ}_{\mathrm{H}}$ exons of these two $\mathrm{KI}$ models were integrated into the mouse $\mathrm{J}_{\mathrm{H}}$, locus, but these models differed from the VRC01 class KI models, in that PGT121 gl$\mathrm{V}_{\mathrm{L}} \mathrm{J}_{\mathrm{L}}$ rearrangements were also integrated into their $\mathrm{J} \kappa$ clusters. Immunization studies in these two models were performed to evaluate the efficacy of two candidate precursor/ reverted PGT121-binding immunogens in particular, 10MUT and 11MUTB. Similar to the reverse engineering approaches used to design the VRC01 class-targeting immunogens $426 \mathrm{c}$ and eOD-GT8, 10MUT and 11MUTB were generated into precursor/gl-PGT121 binders via several rounds of mutagenesis and selection of a native trimeric Env protein, BG505 T332N, which exhibited no appreciable binding to reverted gl-PGT121 Abs (Steichen et al., 2106). In both models, B-cell development appeared to be essentially normal and all B-cells expressed both knocked in $\mathrm{HC}$ and LC rearrangements, consistent with the lack of noticeable (in vitro) poly-/autoreactivity if the PGT121 Ab. As expected, repeated immunization of $\mathrm{GL}_{\mathrm{HL}} 121$ and $\mathrm{Mut}_{\mathrm{H}} \mathrm{GL}_{\mathrm{L}} 121$ mice with native trimeric Env proteins YU2 or BG505 SOSIP, failed to elicit any detectable Ab response, whereas a single immunization with the germline-binding immunogen 10MUT was sufficient to elicit readily detectable 10MUT-binding Abs that were PGT121-like, as interactions with 10MUT were abolished by mutational analysis of the PGT121 epitope.

There are two substantial caveats to these KI models that warrant further investigation. First, the $\mathrm{GL}_{\mathrm{HL}}$ model uses a "least-mutated" member structural inference with key HCDR3 mutations remaining, and thus represents a quasi-intermediate Ab that may have bypassed an earlier selection blockade and/or may not exist in the human repertoire. Secondly, the fact that PGT121 bears a very long HCDR3 makes it probable of having been stringently culled, prior to LC pairing, thus any bona fide PGT121 precursors are likely to be present at exceptionally low frequencies (less than VRC01). Thus, it remains to be seen if bona fide PGT121 precursors can be found in all healthy humans and activated in Kyma mice by 
"N332 supersite" Ab priming-immunogens such as 10MUT. Nevertheless, in conjunction with the priming studies with VRC01 class-targeting immunogens, these initial studies in PGT121 KI models further highlight both the value of Env reverse engineering as an approach to develop potentially efficacious immunogens and human Ig KI mouse models, as lead/translational in vivo platforms for testing their potential.

\subsection{Key issues for bnAb precursor activation and ongoing priming studies in $\mathrm{KI}$ models}

As noted above, significant progress has been made with learning how to prime VRC01-like bnAb development using high affinity precursor-targeting immunogens, and promising initial results also appear to be emerging with those aimed at priming the PGT121 bnAb class. However, as discussed in Section 3.3, an efficacious HIV vaccine will likely need to induce several bnAb classes, for several reasons, including i) achieving adequate cumulative breadth (especially if high SHM levels are truly required, since this will be challenging for vaccination to feasibly recapitulate), ii) minimizing viral escape, and iii) overcoming immunogenetic-mediated response diversity between vaccinated individuals (e.g. due to MHC class II haplotype variation, or for bnAbs with especially long HCDR3s like PGT121, due to repertoire frequencies being potentially so low that not all individuals bear relevant lineage precursors of a particular bnAb class). In this regard, studies to evaluate candidate priming immunogens to various other bnAbs (or bnAb lineages) in several novel bnAb precursor KI models are currently underway, including those in CH103 UCA KI (Williams, Haynes, and Verkoczy, submitted), CH01 UCA KI (Verkoczy \& Haynes, unpublished results), DH270 UCA KI (Alt and Tian, unpublished results), and 2F5 UCA KI (Zhang et al., 2016) or DH512 (i.e. 10E8-like) UCA KI (Alt \& Tian, unpublished results) models, to assess candidate priming immunogens for targeting, respectively: the HCDR3-binder class of CD4bs ${ }^{+}$bnAbs (Gao et al., 2014; Liao et al., 2013b), V2 apex-directed bnAbs (Alam et al., 2013a; Alam et al., 2013b; Andrabi et al., 2015; Gorman et al., 2016), V3 high-mannose patch ("N332" supersite)-specific bnbAbs (Alam et al., 2017), and MPER-specific bnAbs (Alam et al., 2011; Zhang et al., 2016).

With respect to ongoing CH103 UCA KI priming studies specifically (discussed further below), it is noteworthy that the transmitted/founder (TF) virus-derived priming immunogens and the UCA KI model made for evaluating them, have both been engineered based on the B-cell lineage design (Haynes et al., 2012b) of activating and maturing bona fide (time-of-infection) UCAs, which represents an alternative concept to that of reverse engineering discussed above, to prime VRC01 and PGT121 class bnAbs. Both strategies will be discussed further, in the section below describing boosting strategies to drive breadth, and even further in Section 6, regarding their translatability and relevance to vaccine design. Also notable is that priming immunogens made by several groups, based on either strategy, and in various forms (soluble or multimeric gp120s, native or non-native trimers, etc..) are being tested in various new precursor bnAb KI models that are distinct yet related, i.e. expressing either reverted or UCA bnAb rearrangements (or unrearranged segments) of a given bnAb. Additionally, in many cases now, greater than one bnAb KI model is being made for a specific "bnAb class". Thus, the field as a whole is moving towards much more iterative and collaborative immunogen testing in complementary animal models, which 
provides considerable hope that several feasible Env priming targets will be identified fairly soon, a key step in developing an effective bnAb-based vaccine strategy.

While much initial progress has been made in developing priming immunogens, we are still in the early phases of understanding the rules for optimally engaging the spectrum of B-cell lineage precursors with the potential ability to go on and develop neutralization breadth. In this regard, there are at least two key issues we currently must consider in further studies. The first is that despite the demonstration that core proteins synthetically-engineered to bind VRC01 class bnAb precursors at high affinity in vitro can also prime these precursors in vivo, the process appears to be highly inefficient, and in particular, also leads to induction of many "off-target" (cross-reactive) non-bnAb responses, especially in studies done in the more physiological $\mathrm{KI}$ models (e.g. $\mathrm{V}_{\mathrm{H}} 1-2$ mice), and as previously noted in vitro (McGuire et al., 2014). Optimization of bnAb precursor priming/activation relative to these off-target clones, is likely to be a key issue to resolve, since more efficient priming may considerably shorten the prolonged maturation pathways seen during infection, and thus could play a significant role in devising a more feasible vaccine strategy. In this regard, since some native-like trimer immunogens already appreciably bind some of the bnAb precursors e.g. V1V2/glycan class bnAbs (Sliepen et al., 2015), and are now further optimized for their ability to bind bnAb precursors of various classes at higher affinity (in some cases simultaneously), and/or are also being mutlimerized (Andrabi et al., 2015; Gorman et al., 2016), R.Sanders, personal communization). When these immunogens shortly become available, it will be highly relevant to compare these to the bnAb precursor-priming "core protein" antigens noted above, as well as to more "minimal" immunogens (Alam et al., 2013a), both in terms of strength of precursor bnAb induction and relative elicitation of "offtarget" responses. Thus, assuming concepts of high affinity and/or multimerization can be equally applied to re-design trimer immunogens, an active area of interest will be testing the relative effectiveness of such trimers to specifically and robustly engage bnAb precursors, relative to other immunogen forms. In this regard, arguments have been made on both sides as to why such trimeric constructs may or not be better: on one hand, there is the compelling case that closed conformations of near-native trimeric proteins can most effectively present the complex quaternary (CD4bs) and/or glycopeptide bnAb epitopes to precursor BCRs, while most efficiently shielding many immunodominant, off-target (non-neutralizing) epitopes that would normally be occluded (de Taeye et al., 2015; Sanders et al., 2015). On the other hand however, such structures (relative to non-stabilized proteins) could lack flexibility sufficient for optimally engaging $\mathrm{IgM}^{+}$BCRs of naïve (non-activated) precursors. Therefore, such evaluations of stabilized versus non-stabilized trimers, and other protein forms will be interesting and important issues for the filed, likely best answered via empirical testing in KI models.

The second issue that impacts choice of immunogens at the priming phase, is at what stage tolerization occurs in bnAb lineages and more specifically, what is the signaling status of their precursors in the naïve (pre-immune) repertoire? As I touched on in Section 4, anergy of naïve precursors appears to be an issue for many, but not all bnAb lineages. In particular, this phenotype is certainly observed in all MPER ${ }^{+}$bnAb KI models tested thus far (Chen et al., 2013b; Doyle-Cooper et al., 2013; Finton et al., 2013; Verkoczy et al., 2011a), and most likely extends to all MPER ${ }^{+}$bnAbs, based on their in vitro self-reactivities (Liu et al., 2015; 
Verkoczy, Kelsoe and Haynes, 2014); whereas for precursors of bnAbs targeting other Env vaccine targets, this may be case-dependent, as seen for the various $\mathrm{CD} 4 \mathrm{bs}^{+}$bnAb KI models made thus far. Indeed, as already discussed earlier, naïve 3BNC60 GLV $\mathrm{HL}_{\mathrm{HL}} \mathrm{KI}$ mice exhibit anergy amongst other tolerance controls (McGuire et al., 2016)(Fig 6C), as does the CH103 UCA dKI (HC+LC) KI model (Verkoczy, Williams, and Haynes, manuscript submitted))(Fig 6G), whereas all germline/precursor VRC01 KI models mice exhibit unperturbed B-cell development, even though VRC01-expressing B-cells appear to develop signs of self-reactivity after a certain degree of vaccine-induced maturation has occurred, including binding to the candidate self-antigen UBE3E ligase ((Tian et al., 2016) and Tian, Alt, \& Haynes, personal communication). In all these examples of pre-immune bnAb precursor anergy (excepting VRC01), the issue of "off-target" responses to vaccination may be especially problematic, since non-anergic clones cross-reactive to any of a number of non-bnAb epitopes found in Env immunogens are likely to be preferentially amplified over anergic bnAb precursors, especially if local immunogen concentrations are limiting. Thus, aside from reiterating the importance of defining the pre-immune biology (i.e. self-antigen exposure of precursors, prior to immunogen encounter) for each individual bnAb lineage, this also suggests that for those lineages for which B-cell precursors are found to be anergic, issues beyond standard immunogen design (i.e. immunogens needing to bind anergic bnAbexpressing B-cell, due to their lower surface IgM densities, with especially high avidity via multimerization and/or high affinity), such as optimization of their delivery/presentation to naïve B-cells may also be critical. Additionally, as discussed in Section 4, anergic B-cells may have additional signaling requirements that include antigen-independent survival signals, i.e. stronger/more specific CD4 T-cell help and/or TLR signals (Chaturvedi, Dorward and Pierce, 2008; Cooke et al., 1994; Leadbetter et al., 2002; Marshak-Rothstein and Rifkin, 2007; Sekiguchi et al., 2002; Seo et al., 2002), which may also be necessary to incorporate into vaccine regimens. In the case of primed $3 \mathrm{BNC} 60 \mathrm{GLV}_{\mathrm{HL}} \mathrm{KI}$ mice, it is likely additional (non-BCR) signals may be lacking, as multimerization of the VRC01 classtargeting immunogen $426 \mathrm{c}$ can activate and expand $3 \mathrm{BNC} 60^{+}$precursors, yet elicits virtually no SHM (McGuire et al., 2016) relative to other comparable (single) primes in VRC01 or PGT121 KI models. Similar findings have also been observed for anergic 2F5 precursors in 2F5 UA (precursor) dKI (HC+LC) mice, whose priming with an MPER bnAb peptide, when in complex with liposomes (and TLR agonists) can bind the 2F5 UA Ab (but not non-bnAb MPER $^{+}$Abs) at high avidity in vitro (Alam et al., 2011; Zhang et al., 2016) but can only partially activate $2 \mathrm{~F} 5$ lineage precursors in vivo. Specifically, in these mice, KI HC/LC Bcell can be robustly expanded, yet undergo minimal class switch or SHM, even after multiple immunizations (Zhang et al., 2016), thus again suggesting that not only higher avidity BCR interactions, but other signals, need to be incorporated.

Finally, concepts to fine-tune priming immunogens may not just be restricted to triggering anergic bnAb lineages, but may also apply for bnAb precursors not subjected to negative selection. For instance, tweaking timing, amounts and avidity ranges of priming immunogens may be required to further optimize responses, since too high avidities/ concentrations, excessive multimerization, or overly prolonging prime times, could all lead to early exit from GC reactions and/or preferentially induce T-I responses (Benson et al., 2007; Mond et al., 1995). In this regard, extrapolating more narrow, relevant affinity 
"ranges" from in vitro results may be difficult because precursor-targeting immunogen affinities in vitro are against soluble IgGs and thus do not necessarily reflect interactions with $\mathrm{IgM}^{+} \mathrm{BCRs}$ on naïve B-cells in vivo. In such cases, in vitro studies would fail to predict $\mathrm{GC}$ recruitment and $\mathrm{AM}$ in vivo. Indeed, at least in some cases, while prolonged GC maturation is based on selection for clones with higher acquired affinities (Dal Porto et al., 1998; Schwickert et al., 2011; Shih et al., 2002), lower affinity clones have been found to best initiate GC formation, while high-affinity naïve B-cells are recruited for plasmacyte differentiation (Benson et al., 2007; Linton, Decker and Klinman, 1989; Paus et al., 2006; Phan et al., 2006; Raman et al., 2007). In these cases, strategies guiding the bnAb pathways may benefit from high-affinity boosting, but overly strong initial signals may be counterproductive, since activation thresholds would be predicted to be much lower than seen during typical positive selection. The issue of avidity priming ranges could be even more relevant for any bnAb lineages found to be hyper-responsive to BCR ligation, and thus which are not only on the complete opposite end of the spectrum as being negatively selected/anergic, but also having activation requirements substantially differing even from those of "typical" naïve B-cells; in such, cases lower affinity signals could actually be beneficial, as such clones may have ultra-low affinity thresholds for activation. For example, B-cells with a propensity to be non-specifically cross-primed by endogenous or environmental (super) antigens may be hypersensitive to subsequent immunogen priming (Silverman and Goodyear, 2006). Thus overall, the systematic evaluation of precursordirected immunogens of varying avidities for iterative testing in KI models in which representative bnAb lineages exhibit distinct degrees of selection will likely be critical to optimize bnAb lineage priming across multiple targets.

\subsection{Emergence of sequential boosting concepts for broadening nAb responses to HIV}

While the bnAb precursor-targeting immunogens we discussed above have been/are being designed with the goal of activating naïve B-cells and initiating bnAb lineage affinity maturation, repeated immunizations with such priming immunogens alone, would not be anticipated to induce the complete set of somatic mutations required for development of broad neutralization activity (Burton and Hangartner, 2016; Haynes et al., 2012b; Klein et al., 2013; Kwong and Mascola, 2012; West et al., 2014). In this regard, the assertion that immunization with two or more heterologous Env immunogens will generate more broadly reactive humoral responses has been a long-standing premise in the field, yet no such approaches had led to measurably better responses (reviewed in (Bonsignori et al., 2012a; Mascola and Haynes, 2013; McElrath and Haynes, 2010)). As discussed in the previous section, we now know from recent studies that this has been due, at least in part, to not having employed immunogens with the ability to robustly and/or specifically activate bnAb precursors. Furthermore, in these earlier heterologous prime/boost studies, it was additionally unclear if specific combinations of immunogens were required for broadening responses, and if so, which ones gave significant, yet incrementally better responses (i.e. not detectable at the serum Ab level), since technology for probing affinity maturation at higher resolution (by NGS and/or single-cell memory B-cell sorting) has been only relatively recently developed, as have more practical vaccine testing platforms, i.e. the humanized Ig KI models being discussed in this review (where human repertoire complexity can be simplified when required). Over the past four years, this additional gap in knowledge has 
been addressed, as significant rationale now has been obtained for testing the concept of serial administration of multiple heterologous envelopes, and this in turn has currently reinvigorated efforts to develop rational heterologous boosting strategies, and now provides a conceptual framework for testing basic sequential (step-wise) protocols in KI models (as we will detail further below), as starting points for iteratively testing tailored modifications engineered to enhancing $\mathrm{nAb}$ response breadth.

While early versions of "sequential-like" strategies had been tried in the past that not exhibit obvious serological signals of enhanced neutralizing Ab breadth (Eda et al., 2006; Klinman, Higgins and Conover, 1991; Malherbe et al., 2011), but as just mentioned, methodologies needed to probe the vaccine-activated memory B-cell repertoire at a depth where signals of breadth development could be evaluated were not available, nor had relevant Ig locushumanized animal vaccine models been developed to further increase the resolution of bnAb-relevant readouts. Recent developments, however, have now provided strong rationale to carry out more directed sequential (step-wise) strategies, most notably, recent information from structure-function and longitudinal patient time-of-infection sampling analyses, both have been leveraged to develop two distinct types of sequential immunization strategies.

The first is a structure inference-based "reverse engineering" stepwise vaccination concept (Briney et al., 2016; Burton et al., 2012; Escolano et al., 2016; McGuire et al., 2013; Tian et al., 2016; Zhou et al., 2010). In its most general form, this approach involves first using the type of synthetically-engineered immunogens described above for the priming phase, in order to activate naive B-cells to switch and form memory B-cells that have undergone some degree of SHM. The sequential boosting phase that then follows employs a related set of synthetically engineered immunogens, based on introducing structural modifications (e.g. gradually increasing key $\mathrm{N}$-glycosylation sites in the CD4bs region, in order to incrementally resemble native Env. The goal of such "directed" boosting would be to preferentially reactivate memory B-cells expressing Abs that progressively matured toward recognizing more native forms of Env. The process would be reiterated with more native-like Env antigens, with the goal of eventually using native Envs to select out B-cells expressing matured bnAbs. The overall concept of this immunization scheme is thus to guide $\mathrm{Ab}$ maturation gradual stepwise, which imparts more realistic demands on the SHM required for each boosting step. A key related consideration with such strategies, in order to ensure proper shepherding of AM, i.e. towards breadth development, takes place, is to have overlap in the binding profiles of the immunogen sets/pools used in such stepwise regimens, i.e. boosts must include overlapping administration of "bridging" immunogens e.g. those that can bind both precursors and more "intermediately-native" forms, with those which can bind the same intermediate forms, in addition to more (or fully) native Envs. Analogous "reverse engineering" sequential strategies have also been proposed for other highly variable pathogens, and in some cases, such as in efforts to develop a universal flu vaccine, have also begun to produce some successful proof-of-concept results (Corti and Lanzavecchia, 2013; Dormitzer, Grandi and Rappuoli, 2012; Lanzavecchia et al., 2016), albeit, using regimens not as complicated as are likely to be required in eliciting HIV bnAbs, to deal with greater viral diversity. In particular, sequential immunizations with influenza chimeric immunogens comprised of HA domains derived from various head regions, but the same stalk domains have been demonstrated to induce bnAb responses recognizing the conserved HA stalk 
while conferring cross-protection to the various head domains (Margine et al., 2013; Nachbagauer et al., 2014).

The second distinct type of sequential immunization concept developed for broadening the HIV neutralizing Ab response is the patient-based strategy of B-cell lineage design (Haynes et al., 2012b; Haynes et al., 2016; Liao et al., 2013b). In particular, the elucidation of virus and $\mathrm{Ab}$ co-evolutionary molecular pathways from which effective bnAbs evolve during infection, via comprehensive longitudinal sampling of HIV-infected subjects (Bhiman et al., 2015; Bonsignori et al., 2016; Doria-Rose et al., 2014; Gao et al., 2014; Kong et al., 2016; Liao et al., 2013b; Moore et al., 2012; Moore and Williamson, 2016; Moore, Williamson and Morris, 2015; Wu et al., 2015), has not only served as the rationale behind this approach, but has also been a transformative event for highly-variable pathogen vaccinology in general. From such virus co-evolutionary studies, not only has it possible to decipher the molecular basis of the ontogeny involved in bnAb recognition of progressively more evolved Env trimers, but these studies have also provided a natural model of breadth development. Although knowing how much of the exceptional degree of affinity maturation observed during bnAb evolution is actually required during immunization is obfuscated by other, non$\mathrm{bnAb}$ related factors arising from the chronic infection milieu, such natural evolutionary pathways of bnAb development nevertheless serve as a starting blueprint, whose primary goal for vaccination is to re-capitulate the viral evolutionary process using immunogens selected at key evolutionary branchpoints, in order to enable the gradual accumulation of mutations that can steer affinity maturation towards acquiring increased breadth. Longerterm however, the optimization of such strategies would be sought, via further rounds of iterative testing, with the goal of "short-circuiting" the maturation process during natural infection that served as the initial roadmap, in order to achieve a practical boosting regimen, i.e.one refined to only include the minimal boost(s) required to achieve reasonable breadth. The general strategy for the B-cell lineage design vaccine concept is shown, using the wellcharacterized CH103 bnAb lineage as an example ((Fig 6) (Haynes et al., 2012b; Liao et al., $2013 b)$. In this alternative type of sequential vaccination strategy, naïve precursor B-cells expressing the bona fide (time-of-infection) Unmutated Common Ancestor (UCA) of a particular bnAb lineage's evolutionary pathway, would be activated by using the pathway's original Transmitted Founder (TF) virus (instead of priming with a synthetically-constructed immunogen optimized to bind a structurally-inferred reverted bnAb). Sequential boosts would then be done using Env immunogens generated from the viral isolates that co-evolved and were confirmed in vitro to bind at high affinity with its progressively matured $\mathrm{Ab}$ intermediates.

Although the general applicability of this B-cell lineage-design sequential boosting approach in vivo remains to be validated in experimental immunization models (including KI mice) and requires careful comparative evaluation to the "reverse engineering" type of sequential approaches we described above, the premise behind it may have the greatest potential as part of a relevant general vaccine strategy, for two key reasons. First, it is becoming better appreciated that bnAbs obtained from multiple donors, which target similar Env epitope clusters, including both "HCDR3-binder" and "CD4 mimic" classes of CD4bs+ bnAbs (Bonsignori et al., 2016; Scharf et al., 2016; Zhou et al., 2015), and also V2/glycan-directed bnAbs (Andrabi et al., 2015; Gorman et al., 2016) share similar structural hallmarks of 
appropriate "angles of approach" to interact with Env trimers (a key early predictor of having breadth potential), and also use reproducible developmental affinity maturation pathways. Thus, such structural and developmental commonalities make such lineage Abs and their co-evolved viruses good representative templates for vaccine design. Furthermore, Env viral evolution in the original CH505 HIV-infected individual has recently been found to be recapitulated in strikingly similar fashion during infection of rhesus macaques with SHIV505, a SHIV that has been re-engineered to bear the same TF Env sequence isolated from the CH505 individual (Li et al., 2016), suggesting this lineage's co-evolutionary AbEnv pathway trajectories are not only reproducible between individuals but even across primates, thus further validating B-cell lineage design as a viable strategy to guide vaccinemediated development of bnAb breadth.

The two sequential approaches we have described above are certainly not mutually exclusive with each other, nor with alternative sequential concepts, such as using a more "iterative"based boosting approach based on empirical testing after each immunization to inform subsequent boosts, for instance, screening serum from each immunization step for their ability binding to a panel of progressively of related, but progressively evolved Env boosting immunogens, as has recently been described (and will be further discussed below) for inducing PGT121 nAb responses (Escolano et al., 2016). Ultimately, an overall bnAb-based vaccine approach will likely be a hybrid of several approach, based on building from both sequential results and also incorporating other vaccine concepts, such as ones manipulating $\mathrm{GC} / \mathrm{Tfh}$ responses to enhance bnAb affinity maturation, or adjuvanting modifications aimed at overcoming B-cell tolerance roadblocks at various selection stages of bnAb development, either prior to or during boosts as necessary. Additionally, while the sequential boosting approaches for driving breadth are gaining traction in the field based on the rationale discussed above, it will also be key to comparatively evaluate related but distinct boosting approaches proposed for recapitulating diversity, such as concepts that "incremental" boosting, where Env diversity is gradually increased, or "swarm" boosting, where a cocktail of co-evolved Envs in a bnAb maturation pathway (or even more generally, from multiple donors, representative of the natural viral diversity seen in several similar lineages) are administered simultaneously (Haynes et al., 2016). The assertion that sequential boosting may be superior (relative to additive or swarm boosting approaches) in guiding bn Ab maturation towards breadth is supported by recent bioinformatic modeling studies (Shaffer et al., 2016; Wang et al., 2015). However, it could also be theoretically argued that the antigenic diversity swarm/additive approaches confer is beneficial, rooted on notions extrapolated from both the above viral-Ab co-evolution studies and central tenets of B-cell affinity maturation studies, namely that: i) activating B-cells of varying affinities in an oligoclonal family may increase the pool to generate high-affinity memory B-cells, and ii) HIV-1 quasi-species continuously evolve during infection, and as such Env variants may not be generated in a perfectly sequential manner, but as overlapping pools of related Envs. Ultimately, empirical comparisons of these related yet distinct approaches (and combinations therein), enabled by more iterative testing should provide clarity to the issue of if and which approaches are most effective in breadth induction.

Below, we will review results from recent sequential boosting studies in precursor bnAb KI models, which all show promising initial results, and thus provide beachheads to now test 
what improvements are now needed to drive breadth, relative to (and/or in combination with) other vaccine approaches. In reviewing these studies, the KI strains being used will themselves be discussed, both in terms of how they provide unparalleled iterative platforms to test such boosting strategies, but also regarding their current limitations for informing the most relevant immunogen design. In subsequent sections, these limitations will themselves be further discussed, as will ongoing efforts to improve KI model design, to allow even more practical and physiological vaccine testing.

\subsection{Evaluation of sequential (stepwise) boosting approaches in bnAb KI models}

Several sequential boosting strategies (both reverse-engineering and patient-based) have either been recently reported or are currently underway for numerous bnAb classes and in various other KI models, including those for studying various V1V2glycan nAb class boosting regimens, being done as several collaborative efforts between the Scripps/Duke CHAVI-ID consortiums, the NIH-Vaccine Research Center, and Cornell/Amsterdam. In this section, we will review promising initial results from vaccination studies in various KI models in which candidate sequential boosting regimens for broadening bnAb responses have been evaluated. As for the previous sections detailing priming studies in these models, we will primarily focus on studies that have examined the breadth of VRC01-like nAb responses to sequential vaccination, thus far all using synthetically-engineered immunogens to boost various $\mathrm{V}_{\mathrm{H}} 1-2$ germline precursor KI models. However, some very new findings in PGT121 KI mice, involving a promising sequential boosting strategy to drive breadth of V3 high mannose patch-directed nAbs will also be reviewed, as will a just-completed study with the "4-valent" CH505 sequential vaccine regimen (Fig 6), devised to induce "HCDR3binder" CD4bs-class nAb responses in CH103 UCA KI mice, the first patient-based (B-cell lineage design) concept to be evaluated in vivo.

\subsubsection{Sequential-based induction of CD4bs nAb responses in VRC01 \& $\mathrm{CH} 103$} class precursor KI models-As the priming studies in the VRC01 gH, $3 \mathrm{CBN} 60 \mathrm{GLV}_{\mathrm{H}}$, and $\mathrm{V}_{\mathrm{H}} 1-2 \mathrm{KI}$ models (Figs 5A,B,E) have all demonstrated, immunogens targeting VRC01class precursors can successfully activate and initiate detectable SHM in $\mathrm{V}_{\mathrm{H}} 1-2 \mathrm{KI}$ expressing naïve B-cells, and elicit VRC01-like Abs to mature enough to attain some neutralization activity (Dosenovic et al., 2015; Jardine et al., 2015; Tian et al., 2016). However, even repeated immunizations with such priming immunogens, alone, fail to induce the complete, substantial set of somatic mutations required by this bnAb class for their broad neutralization activity. Given the limited SHM and breadth priming elicited in these models, VRC01-like Abs at this stage would be unlikely to effectively recognize, and thus be boosted by, native Env proteins. Indeed, as noted above for the version of the 3BCN60 KI model generated to represent a synthetic 3BNC60 "later intermediate" (Fig 5D), immunization with either the native Env trimer BG505 SOSIP or the priming immunogen eOD-GT8 60mer could expand and promote further maturation of this intermediate, but of the tow immunogens, BG505 SOSIP induced considerably broader neutralization (Dosenovic et al., 2015), suggesting later intermediate Abs if they can be elicited, can be best driven to complete maturation by native-like Env immunogens. Taken together, these considerations thus prompted the development of "bridging" immunogens for the initial boosting phase, to promote further maturation of VRC01-like primed Abs (Briney et al., 
2016). Two such immunogens were produced as initial boosting components of candidate sequential regimens. One construct, BG505 core GT3, was multimerized using the same nanoparticle platform as the precursor-targeting eOD-GT8 immunogen, but unlike eODGT8, contained the complete gp120 core of BG505 Env including its inner and outer domains. Additionally, BG505 core GT3 contained fewer mutations than eOD-GT8 to make the CD4bs more "native-like", including retaining three critical N-linked glycosylation sites, resulting in this construct binding mature VRC01-class Abs with $>1000$-fold higher affinity than their germline-reverted counterparts. As an alternative bridging immunogen, BG505 GT3 was also produced as a native Env trimer, i.e. BG505 GT3 SOSIP.

To test the efficacies of these two bridging immunogens, VRC01gH mice, one of the $1^{\text {st }}$ generation "reverted" KI models described above under the priming studies (expressing $\mathrm{V}_{\mathrm{H}} \mathrm{DJ}_{\mathrm{H}}$ rearrangements bearing germline $\mathrm{V}_{\mathrm{H}} 1-2$ linked to the mature HCDR3 from the original VRC01 bnAb; Fig 5A), were immunized with two sequential immunization protocols (Briney et al., 2016), differing only in the two bridging immunogens described above. For both sequential schemes, the mice were primed with eOD-GT8 60mer, then boosted once with the bridging immunogen (i.e. BG505 GT3 core multimers or BG505 GT3 SOSIP), and finally boosted twice with BG505 SOSIP N276, a further modified, (even more native) BG505 trimer lacking only the N276 N-linked glycosylation site. Adjuvant (Ribi) was used during the prime, while boosts were either done in its presence or absence. As with the priming studies discussed earlier, both sequential immunization protocols elicited CD4bs + Abs comprised of VRC01gH and mouse LCs bearing 5-aa CDRL3s, with the regimen that included BG505 core GT3 regimen in particular eliciting the highest frequency of activated (IgG-switched) memory B-cells, with substantially more SHM induced (with up to $16 \%$ mutation rates in HCs observed) in all animals from this immunization group. Importantly, there was also evidence for sequence convergence of 5aa CDRL3-containing LCs induced, with some mutations corresponding to those found in VRC01-class bnAbs known to be critical for gp120 binding. Particularly striking was a strong enrichment for a conserved glutamic acid residue at position 96 within the 5-aa CDRL3, with some CDRL3s even having acquired the complete consensus QQYEF motif of VRC01-class bnAbs. IgG Abs purified from serum of mice administered the entire sequential boosting regimen did not neutralize any isolates from a panel of eight WT viruses, but when the critical N276 residues of these viruses were mutated, the sequential immunization-elicited IgG Abs could neutralize multiple tier $2 \mathrm{HIV}-1$ isolates bearing the N276 mutation. As with those assayed with IgG serum Abs, viral strains were also refractory to neutralization by mAbs cloned from immunized mice, while multiple N276-mutagenized viral strains were not, although interestingly, two mAbs in particular neutralized a tier 2 virus N276 mutant 191084 B7-19, with comparable potency as the mature VRC01 $\mathrm{Ab}$, and could also weakly neutralize the native form of this viral isolate, i.e. bearing the intact N276 glycosylation site. Thus, along with inducing substantial SHM, this boosting regimen matured VRC01-like Abs to an "intermediate" stage, since vaccine-elicited serum nAbs (and most mAbs isolated) could not bypass the glycan 276 "bottleneck".

Sequential vaccination for inducing breadth of $\mathrm{CD} 4 \mathrm{bs}^{+} \mathrm{nAb}$ responses was also very recently evaluated in a collaboration between the Alt lab and the NIH Vaccine Research 
Center, using the $\mathrm{V}_{\mathrm{H}} 1-2$ unrearranged " $2^{\text {nd }}$ generation" KI model (Fig 5E), already described above under the priming studies. Due to the diverse Ab repertoire in this model, VRC01-like precursors were highly infrequent, making the affinity maturation studies impractical. To facilitate such studies, a model, $\mathrm{V}_{\mathrm{H}} 1-2 / \mathrm{LC}$ was generated via introduction of a rearranged gl-VRC01 LC integrated into the J $\kappa$ locus of the $\mathrm{V}_{\mathrm{H}} 1-2$ model (Fig 5F) so that higher frequencies of precursors could be generated. A similar but somewhat more complex sequential immunization strategy was used to vaccinate the $\mathrm{V}_{\mathrm{H}} 1-2 / \mathrm{LC}$ model. eOD-GT6 60mer (an earlier version of eOD-GT8 with lower binding affinities to the germline-reverted VRC01 Abs) was administered to selectively activate B-cells expressing VRC01-like precursor Abs. The first set of boosting immunogens was based on the Env protein of $426 \mathrm{c}$ virus, with the use of Env antigens from a different viral strain used for this boost protocol (than from which the priming immunogen eOD was derived from) added as a consideration, with the intent of helping to focus immune responses to CD4bs. The 426c gp120 core (a similar concept to the "BG505 GT3 core" described above for the Scripps study), with mutations at three key N-glycosylation sites (N276, N60, N463) was then used as a bridging immunogen in initial boosts. At this early stage of immunization, the precursor Ab would be expected to have insufficiently progressed through AM, and thus the triple glycosylation mutation in this immunogen was intended to facilitate interaction with early intermediates. In particular, and analogous to the strategy used with BG505 GT3 core, the 426c core immunogen contains both the outer and inner domains of gp120, whereas eOD-GT6 consists primarily of a minimal outer domain, thus the 426 core bridging immunogen was intended to serve as a first step in shepherding Abs towards interacting with the CD4bs in a more native context. Subsequent boosts, devised to gradually select for B-cells expressing nAbs capable of accommodating CD4bs-proximal glycans were then carried out by stepwise administration of $426 \mathrm{c}$ core derivatives engineered to have either two, one, or none of the three key $\mathrm{N}$-linked glycosylation site mutations. The final boost then employed the native trimeric form of $426 \mathrm{c}$ Env protein.

As with the sequential studies in the VRC01 $\mathrm{gH}$ model, this more complex (six step) sequential protocol elicited CD4bs-specific VRC01-like Abs, which in this case were composed of $\mathrm{KI} \mathrm{V}_{\mathrm{H}} 1-2 * 02 \mathrm{HCs}$ and gl-VRC01 LCs. This stepwise regimen also successfully induced Abs that accumulated substantial levels of SHM throughout the course of immunizations, including developing some mutations which mimicked those in matured VRC01-class Abs. Interestingly, LC mutations were heavily focused on the CDRL1, a region critical for avoiding steric clashes with carbohydrates at the Loop D N276 glycosylation site. Notably, serum VRC01-like Abs from mice receiving the full stepwise vaccine regimen exhibited neutralization activities against various autologous $426 \mathrm{c}$ viruses lacking one or more of the various $\mathrm{N}$-glycosylation sites, as well as a heterologous virus lacking N276, but not against the WT (fully-glycosylated) Tier 2 426c virus. The mAbs cloned from these immunized animals largely recapitulated the serum binding data, with 12/15 Abs isolated exhibiting neutralization activities against the autologous 426c virus with N276 mutation, some with potencies comparable to that of the mature VRC01 bnAb, and 4/15 Abs neutralized a heterologous virus that lacks N276 glycosylation. Additionally, 1/15 mAbs also bound (albeit weakly) to the heterologous native BG505 Env trimer, similar to potentially promising preliminary results seen in the other sequential study, again suggesting 
in rare cases (below detection limits in serum assays), some clones could circumvent the carbohydrate-related issues at N276. Thus, as with the above sequential vaccination study by the Scripps group in the VRC01-gH mice, along with inducing substantial SHM, this boosting regimen matured VRC01-like Abs to an "intermediate" stage, at which point vaccine-elicited serum nAbs (and most mAbs isolated) could not bypass a key glycan bottleneck. Nevertheless, more generally, these two initial studies provides validation for both the strategy of sequential boosting (as part of a heterologous immunization concept) and the KI mouse model for evaluating maturation of VRC01-like Abs, as viable approaches for studying vaccine-guided breadth development and provide beach-heads for iterative optimization of this strategy.

Even more recently, a sequential vaccine regimen targeting the "HCDR3 class" CD4bs + bnAb lineage, CH103 in both rhesus macaques and in a CH103 UCA het dKI mice (Fig 5G), a model that heterozygously expresses the $\mathrm{HC}+\mathrm{LC}$ rearrangements of the bona fide (timeof-infection) CH103 UCA Ab (W. Williams, L. Verkoczy, B. Haynes, manuscript submitted), and also only replaces a portion of the J火 cluster, to allow for additional secondary LC events to occur. These features combined were intended to decrease precursor frequencies, so that $\mathrm{AM}$ to this vaccine regimen could be studies in a highly competitive setting. As noted in Section 4.3, very low $(<1 \%)$ frequencies of $\mathrm{HC}+\mathrm{LC} \mathrm{KI} \mathrm{CH103} \mathrm{clones}$ were found in the naïve mature B-cell repertoire, due to extensive $\mathrm{HC}$ and LC editing. As in the model, multimerization could activate the residual, in this case of het mice, very minor numbers anergic B-cells ex vivo, and also like that study, priming gp120 immunogen could not expand, but induce class switching memory B-cells. However, using a "4-valent" B-cell lineage sequential strategy (Fig 6), even in this setting, with a pre-immune repertoire of bn $\mathrm{Ab}$ precursors that was anergic, can still preferentially drive their initial expansion and some degree maturation (relative to repeated priming TF immunogen). However, while axctivation and bnAb lineage initiation with sequential immunization was better than with priming alone, in this setting am was highly limited, and sequential immunization and lineage-specific responses became diverted very early in this regimen (after 2 boosts), indicating either better T-cell help and or adjuvanting to promote bnAb GC responses and/or increasing precursor frequencies is needed.

Thus, several types of CD4bs-targeting sequential immunization studies, now done in five types of mouse models, have thus far provided several important insights and pointed directions for future studies aimed at optimizing sequential strategies to enhance breadth of $\mathrm{CD} 4 \mathrm{bs}+\mathrm{nAb}$ responses. Most generally three concepts have come to light: 1) activation of precursors require higher affinity immunogens, as had been predicted, 2) several sequential administrations with progressively more native-like Env immunogens are required to mature precursor VRC01-like Abs toward nAbs, since repeated immunizations are ineffective at inducing AM, and 3) "off-target" (non-CD4bs bnAb) responses also occur, but become more prevalent as system polyclonality is increased (for example the VH1-2 KI model, relative to the VRC01 gH model), indicating either priming immunogens are not that highly selective for bnAb precursors, or bnAb precursors eventually get outcompeted by some cross-reactive clones over time. With respect to the CH103 UCA dKI model specifically, like the $\mathrm{V}_{\mathrm{H}} 1-2 \mathrm{KI}$ model, it also represents a "quasi-polyclonal system", and like all other four studies in VRC01-like KI models, sequential immunization also induced qualitatively better responses 
than did repeated immunization with the priming immunogen. However, in this case, offtarget responses began to predominate in even more pronounced fashion than the $\mathrm{V}_{\mathrm{H}} 1-2$ model, i.e. after two immunizations, and regardless of vaccine strategy. Also, since the CH103 UCA dKI model was not like the VRC01 models (which exhibited normal B-cell development), and more analogous to the $3 \mathrm{BNC} 60 \mathrm{GL}_{\mathrm{H}} \mathrm{L} \mathrm{KI}$ model, in that preimmunization a minor (non-edited) subset in the periphery of lineage-specific anergic clones existed, the earlier termination of sequential-driven bnAb lineage development in this model most likely reflects more stringent out-competition of anergic bnAb clones, by non-anergic Env cross-reactive clones. This suggests sequential immunization for bnAb precursors like $\mathrm{CH} 103$ (and 3BNC60) while required, is not sufficient to drive proper maturation of bnAb precursors, and additional signals that increase B-cell survival and/or T-cell help will likely need to be incorporated into such regimens.

With respect to inducing VRC01 class of bnAbs specifically, the additional, main common theme from the studies done in all four models is that accommodation of $\mathrm{N} 276$ glycosylation appears to be a bottleneck for VRC01-like Ab development. In mature VRC01 Abs, CDRL1 truncation or mutation helps to accommodate the N276 glycan, as some of the elicited Abs from the immunized VRC01 mouse models utilized mouse LCs with short CDRL1s (Briney et al., 2016; Dosenovic et al., 2015; Jardine et al., 2015; Tian et al., 2016), but this was not enough to overcome this bottleneck that arises after partial completion of maturation.

\subsubsection{Sequential vaccine broadening of V3-glycan "N332 supersite" nAb responses in PGT121 KI models-In addition to the sequential immunization} strategies like those described above for $\mathrm{CD} 4 \mathrm{bs}^{+}$bnAbs, which are based on either structural and/or patient-based information regarding how bnAbs interact with conserved viral epitopes, other, more iterative "empirical" strategies have also been applied as rationale for guiding maturation of precursors from other bnAb classes towards breadth. One such case is a strategy that Escalano et al. have recently devised for the "N332 supersite" class of PGT121-type Abs (Escolano et al., 2016). In this study, the efficacy of such an alternative "iterative" stepwise boosting regimen was evaluated for its ability to generate breadth development of PGT121-type Abs, in the same two KI models, GL $\mathrm{HL}_{121}$, and $\mathrm{Mut}_{\mathrm{H}} \mathrm{GL}_{\mathrm{L}} 121$, which have already been described in Section 5.3. The overall strategy of this iterative approach was to screen serum from immunized animals after each step in this sequential regimen, for their reactivity against gradually less-mutated Env variants and lower affinities for gl-PGT121 (i.e. 7MUT, 5MUT, 3MUT, etc..) which are derivatives of the nearnative WT BG505 SOSIP trimer, T332 that represent progressively more "native-like" AM intermediates between the 10MUT priming immunogen, and BG505 Env (Steichen et al., 2106). Essentially, the least modified Env variants that exhibited highest serum binding affinity after each round of vaccination was then used in the subsequent boost.

Thus, after successfully engaging and inducing initial maturation of PGT121-like Abs with the synthetically-engineered priming immunogen 10MUT, as noted above (in Section 5.3), the serum acquired binding affinity for an engineered version of BG505 Env (7MUT) with fewer mutations, thus closer to native Env, than 10MUT. Accordingly, trimeric 7MUT SOSIP was then used for the second immunization to guide AM toward recognition of more native Env forms. After second immunizations, serum Abs gained detectable affinity toward 
an even less mutated forms of BG505 Env, 5MUT, which was then chosen as the third boost. After third immunizations, binding activity for the autologous WT BG505 SOSIP T332 Env trimer as well as various other heterologous native Env trimers became detectable in serum, leading to the use of the native Env trimer as the fourth boost. Finally, with the intent of further broadening serum Ab responses, a "cocktail" of the heterologous native-like WT BG505 Env trimers with diverse variable loop sequences was used as the final boost (done for the two immunizations).

Importantly, at the end of this iterative series of sequential vaccinations, impressive crossclade viral neutralization activities were detectable in serum IgGs from 3/7 GL $\mathrm{HL}_{12} 1$ mice and $7 / 7 \mathrm{Mut}_{\mathrm{H}} \mathrm{GL}_{\mathrm{L}} 121$ mice. To more comprehensively evaluate the degree of neutralization breadth the elicited Abs exhibited, $40 \mathrm{mAbs}$ were isolated and assessed on a panel of 54 tier 2 viruses and 2 tier $1 \mathrm{~B}$ viruses, compared side-by-side with the original (fully matured) PGT121 bnAb. mAbs from $\mathrm{GL}_{\mathrm{HL}} 121$ and $\mathrm{Mut}_{\mathrm{H}} \mathrm{GL}_{\mathrm{L}} 121$ mice were able to neutralize 12 and 23 viruses in the test panel, respectively; whereas in comparison, the original PGT121 bnAb could neutralize 50 viral isolates. Also notably, the neutralizing activities of the cloned mAbs correlated with Along with the development of neutralization activities, the elicited Abs accumulated substantial levels of SHM, some of which mimicked those in PGT121class Abs. Furthermore, repeated immunization with the 10MUT immunogen, or a simple heterologous prime/boost, i.e. 10MUT prime, followed by a boost with WT BG505 SOSIPs, both failed to elicit serum neutralizing Abs or yield cloned mAbs having any significant neutralizing activity, thus validating the specific effect of this particular sequential regimen in inducing the breadth of $\mathrm{nAb}$ responses observed.

Despite the caveats with this particular animal model (further detailed in the next paragraph), this is nevertheless the first proof-of-concept study that shows development of broadly neutralizing responses, from a less mature point in an evolutionary pathway, can be elicited by vaccination. Additionally, together with the studies for eliciting VRC01 class $\mathrm{nAb}$, it also further highlights the general requirement for a directed heterologous prime/ boosting approach to guide relevant bnAb maturation, and more specifically, further validates the "sequential" immunization concept, as a strategy to induce bnAb responses. However, going forward, the induction of PGT121-like Abs not only faces some immunological challenges in common with those for VRC01-targeting bnAbs and other bnAb classes, but also has its own technical issues regarding the particular KI models in these initial studies, that also cannot be overlooked.

Regarding the common issues first, such sequential immunization strategies will need to be able to elicit bnAbs in a complex unrearranged Ab repertoire, where the bona fide PGT121 (UCA) precursor $\mathrm{Ab}$ frequencies after undergoing pre-antigenic $\mathrm{V}(\mathrm{D}) \mathrm{J}$ recombination and $\mathrm{HC} / \mathrm{LC}$ pairing in a fully polyclonal setting would normally result in extremely low frequencies. This problem is likely to be more acute for PGT121-class Abs and other long HCDR3-expressing bnAbs, than for VRC01. PGT121-like HCDR3s play a critical role in this bnAb class' interactions with Env; and for this reason, PGT-121 like Abs have stringent requirements for both HCDR3 sequence and length. By comparison, VRC01-class Abs are more flexible with respect to HCDR3 characteristics, and their more typical HCDR3 length and composition are not normally associated with negative selection-mediated culling that 
long HCDR3-expressing B-cells often face early in B cell ontogeny both prior to BCR expression likely (at least partly) due to both pre-antigenic HC/surrogate LC and HC/LC pairing issues (Ivanov et al., 2005; Martin et al., 2003; Vettermann and Jack, 2010; von Boehmer and Melchers, 2010) and/or due to potential self-reactivity due to their surface expression at the pre-BCR stage (Keenan et al., 2008; Minegishi and Conley, 2001) and the first tolerance checkpoint upon their expression as $\operatorname{IgM}^{+}$BCRs (Meffre et al., 2001; Shiokawa et al., 1999). Thus, the frequency of bona fide precursors of PGT121-like bnAbs would be anticipated to be even lower than those that have been found for VRC01-class Abs (Jardine et al., 2016a; Sok et al., 2016).

Immunization in a fully polyclonal B-cell repertoire poses yet another difficulty: the induction of multiple "off-target" Ab responses by irrelevant (non-neutralizing) epitopes to more complex, multi-epitope immunogens such as Env. In the case of VRC01, the problem can be ameliorated at least in part by using a minimal outer domain where the antigenic variable loops are trimmed away. This solution may not apply to PGT121 immunogens, since V1 and V3 are part of the bnAb epitope. Furthermore, V1-V3 loops mediate protomer association (Julien et al., 2013; Pancera et al., 2013), and as shown by immunization studies in the PGT121 KI models, trimeric but not monomeric immunogens are necessary to elicit adequate immune response (Escolano et al., 2016). Thus, despite the fact that such native trimeric immunogens may better occlude many normally hidden immunogenic epitopes than monomeric gp120 Env immunogens (de Taeye, Moore and Sanders, 2016; Sliepen and Sanders, 2016), even these will present immunogenic non-neutralizing epitopes. For example V1-V3 loops need to be preserved in the trimeric PGT121 immunogens, but the presence of these variable and potentially antigenic loops may increase the chance of diverting immune responses. In this regard, the presence of multiple "off-target" competing clones in the setting of a polyclonal repertoire means that what are anticipated to be very rare PGT121 precursor clones will need optimization of priming immunogens for their ability to bind these precursors at much high affinities than 10MUT currently binds glrevrted PGT $121 \mathrm{KI}$ cells; indeed despite successfully triggering PGT121 Ab responses in vivo in the setting of the $\mathrm{GL}_{\mathrm{HL}} 121$ model, the fact $\mathrm{GL}_{\mathrm{HL}} 121 \mathrm{KI}$ B-cells could not be activated ex vivo (Escolano et al., 2016) lend support to this notion.

Thirdly, while these initial studies in the PGT121 KI model, along with the other ones discussed in this section, are still very much at the proof-of-concept stage, longer term, it will likely be key to learn if and how to "short-circuit" such maturation pathways, as reducing the number of sequential boosting steps required will be critical for devising a more practical and truly feasible global vaccine concept. In this regard, what is clearly the "common theme" in all sequential studies done in KI models thus far (VRC01, CH103, PGT121 class) is that repeated immunization with the same priming immunogen clearly fails to produce any significant nAb responses or SHM, and heterologous prime/boosting with additional Env variants is needed. However, what minimum number of related Envs is needed for guiding such responses appropriately (and if having a limited set is even possible for certain bnAb lineages/classes with substantial SHM accumulated during infection), are key open questions for the vaccine field. The studies thus far suggest multiple Env variants will needed, at least with current iterations of the sequential concepts, and especially for driving complete bnAb evolutionary pathways, in more physiological settings. For instance, 
the sequential study in the VRC01 $\mathrm{gH}$ model that went to an intermediate stage (Briney et al., 2016) required less boosts ( 3 boosts over 4 immunizations), but this model was also more enriched for VRC01-ilike precursors and did not have to deal with the issue of large numbers of competing "off-target" clones, whereas in the more physiological $\mathrm{V}_{\mathrm{H}} 1-2$ model, where there were considerably more off-target responses, a more complex reverseengineering sequential sequential approach that required seven steps achieved similar "intermediate" maturation pathways having limited neutralization breadth (Tian et al., 2016). Also, of the two PGT121 KI models more representative of the PGT121 precursor $\left(\mathrm{GL}_{\mathrm{HL}} 121\right)$, the iterative sequential approach just discussed required five steps (six immunizations) to achieve a heterogeneous and partial degree of breadth, and even in this more "precursor-like" version of PGT121, it is not clear if and at what maturation stage of the evolutionary clade derived from a bona fide PGT 121 UCA these germline-reverted precursors truly represent in the priming/boosting process (see below for further discussion). Finally, the "4-valent" patient-based B-cell lineage design approach in CH103 UCA dKI mice could select for and induce limited maturation of the CH103 UCA (above and beyond repeated immunization with the priming immunogen), but will likely require further immunogens and/or adjuvanting to overcome this block in early maturation. Thus, while the PGT sequential strategy (and the others discussed in this section) represent promising early leads and the most direct evidence that reverted bnAbs can be activated and matured by vaccination, in more physiological settings, where complete maturation pathways need to be induced, such approaches would appear to require considerably more immunization-guided $\mathrm{AM}$, and thus barring more straightforward ways to bypass developmental bottlenecks or short-circuit pathways to relevant breath, it is anticipated much more iterative optimization will be necessary.

With respect to the partial breadth induced by iterative sequential boosting in the GL $\mathrm{HL}^{121}$ KI model in particular, there are also three substantial caveats with this particular model, that give pause to drawing any general, physiological conclusions yet, but definitely warrants further investigation of this promising approach. First, as already mentioned in the priming section, GL $\mathrm{HL}_{121}$ mice use a "least-mutated" member structural inference with key HCDR3 mutations remaining, which represents a "quasi-intermediate" Ab that may or may not normally be induced by affinity maturation of the available human repertoire (either in response to infection or vaccination). Thus, it remains to be seen if bona fide PGT121 precursors can be found in all healthy humans and activated in Kymab mice by "N332 supersite" Ab priming-immunogens like 10MUT. Secondly, in addition to the point mentioned above (that PGT 121 Abs, when knocked-in as both $\mathrm{HC}+\mathrm{LC}$ KI rearrangements likely bypassed negative selection prior to self antigen encounter that HCDR3 issues impart on HC/LC pairing (Vettermann and Jack, 2010; von Boehmer and Melchers, 2010), by also having mutations in the HCDR3, this least-mutated PGT 121 precursor, relative to a bona fide unmutated HCDR3-expressing PGT121 UCA precursor, may also have avoided initial BCR encounter with self-antigen. Thus, immunizations could be done instead in Kymab mice or either newer unrearranged models, either ones based on the " $\mathrm{V}_{\mathrm{H}} 1$-2-like" KI concept, but which has a human D also knocked-in and thus doesn't rely on shorter mouse D segments to generate longer HCDR3s in PGT121-like bnAbs (for which it is nearimpossible to de-convolute SHM versus VDJ recombination contributions), or pre- 
rearranged V(D)J UCA KI models like the CH103 UCA dKI platform, constructed from bona fide time-of-infection patient samples that can allow higher probability inferences of such long HCDR3 junctions to be made. In the next section, we will discuss such newer models being planned in further detail, both in terms of their importance for further bridging the gap between physiological relevance and practicality of the KI platforms we described here (Fig 3). Finally, it is also important to point out that only some of the sequentiallyimmunized GL $\mathrm{HL}_{\mathrm{HL}} 121$ mice (3/7) developed cross-clade neutralization, showing this strategy not only induced partial breadth (12/50 isolates neutralized by mAbs from these mice, relative to 50/50 by the original PGT121 bnAb) but also exhibited variability in developing such breadth, despite the inbred genetic backgrounds of the mice. Thus, these results also suggest immunogens cannot (yet) guide the responses of this partial maturation pathway in highly-reproducible fashion, and thus has to be further optimized and/or combined with emerging patient-based lineage concepts, assuming data becomes available data that potentially could demonstrate reproducible natural infection maturation pathways and coevolved viral isolates that could be used as immunogen templates. One current example of this possibility, as discussed earlier, is new data from an NHP study demonstrating striking resemblance in virus-Ab evolution upon infection with a SHIV, engineered to express the same TF Env as the original CH505 patient (Li et al., 2016).

\section{Ig KI-related methodological advances relevant for studying bnAb development}

Despite the various methodological advances in bnAb discovery, cloning, and characterization noted above, as well as the initial vaccine strategies in various precursor bnAb KI models that have shown promising beacheads (both for priming and inducing a degree of maturation towards breadth), fundamental gaps in knowledge and technical aspects of learning how to induce protective HIV Ab responses remain, both which can benefit tremendously from further improvements in existing human Ig KI methodologies. Below, we will briefly discuss some of these murine gene-targeting methodologies, both established and new alike, that could be applied specifically for this key goal. We will focus on advances that will enhance throughput for generating HIV Ab KI models, further balance their practicality and physiological relevance, and manipulate timing or frequency of bnAb expression or general B-cell selection pressure and SHM levels, which collectively should allow more iterative and practical evaluation of new HIV vaccine candidates for their ability to induce protective $\mathrm{Ab}$ responses, as well as more precise and systematic identification of what the minimal requirements for (and limitations to) developing more durable, robust, and rapid bnAb (or other protective humoral responses). Collectively, these enhancements to KI methodologies should thus ultimately enable relevant refinements to be made for improving protective humoral responses by candidate vaccine regimens.

\subsection{Technologies for high-throughput production of bnAb KI models}

Technical advances for generating relevant humanized Ig KI models in a less costprohibitive and rapid fashion would have the obvious benefit of providing a larger spectrum of models in which protective responses to different Env targets could be more conveniently tested. As a result, increasing the capacity to produce HIV Ab KI models would have major 
ramifications from both a technical vantage-point and more fundamentally, for testing hypotheses relevant to understanding limitations to bnAb induction that exist for vaccination. From the more pragmatic aspect of HIV vaccine testing specifically, as greater numbers of bnAb lineages now begin to be deciphered with time-of-infection sampling, and particularly if similar, re-producible bnAb/viral co-evolutionary intermediates as has recently been reported for $\mathrm{CH} 103$ ( $\mathrm{Li}$ et al., 2016) and could thus be used as templates to shepard bnAb maturation during vaccination, increased numbers of such KI models will be needed that make it more feasible for multiple immunogens and/or adjuvant modifications targeting individual bnAb lineage to be evaluated for their ability to reproduce, and possibly even short-circuit such pathways. For example, more practical and efficient initial readouts like serum neutralization analysis, rather than mAb generation/characterization could be done in such models, and also in more standardized fashion, since direct comparisons to each would be possible. Additionally, because many novel vaccine concepts will mandate some degree of empiricism (for example, in testing multiple timing permutations of when adjuvants/small molecule inhibitors would be best incorporated into regimens to enhance Bcell survival, enhance $\mathrm{T}_{\mathrm{FH}}$ function, or prolong $\mathrm{GC}$ reactions, or for determining the most effective orders of sequentially-administered immunogens during boosting regimens), a much larger array of vaccine regimens targeting individual bnAb lineages could be evaluated at much higher resolution, which collectively would allow more systematic stratification of which new strategies could potentially best elicit candidate protective humoral responses to HIV.

From the basic science side, as noted earlier in this review, a fundamental question for HIV vaccinology is whether certain Env targets are more tractable for vaccination to elicit bnAbs (or "later intermediates" of bnAb lineages) to, either due to requiring lower amounts of affinity maturation and/or not being under tolerance controls. As for the practical issue we discussed above, this question will also require generating significantly larger volumes of gene-targeted animal models, to enable systematic, high-resolution identification of which particular impediments are common or unique at distinct Env targets, and then, devising strategies with modified or specifically tailored immunogens adjuvants, small molecule inhibitors, or T cell primes, in order to test how to overcome them safely. Thus, determining relative tractability of such targets for vaccine candidates will require comprehensive testing of tolerance prevalence and minimal SHM requirements of bnAbs across all Env targets, which in turn will necessitate use of higher-throughput KI-producing methodologies. Given both these practical and basic challenges mandating increased numbers of studies to induce bnAb lineage development, what methods could increase the capacity to make platforms expressing bnAb lineage members in vivo?

One key technological advance with respect to increasing capacity of generating HIV Ab KI models comes from the Alt lab, who applied a methodology they pioneered over 20 years ago, termed RDBC (RAG2 deficient blastocyst complementation) (Chen et al., 1993) originally employed to evaluate the impact any given gene (lymphocyte-specific or generally expressed) had on lymphocyte function and/or differentiation (Chen, 1996; Chen et al., 1994). As proof-of-concept, they recently applied this technology to generate the $\mathrm{V}_{\mathrm{H}} 1-2$ series of models (Tian et al., 2016) that was discussed in the previous section. As was the case for generating these KI models (Fig 7), recombinant ES cells are microinjected into 
Rag2 deficient blastocysts. As RAG2 is critical for V(D)J recombination, all the B and T lymphocytes in the resultant chimeric mice will originate from the injected ES cells. For this reason, the chimeric mice can be directly used for immunization experiments, thus obviating the need for lengthy and costly mouse breeding during germline transmission. The RDBC approach is particularly advantageous for mouse Ig KI models originating from ES cells contain multiple genetic modifications on different chromosomes, which would normally segregate during mouse breeding, such as in the case of bnAb precursor ${ }^{+} \mathrm{KI}$ ES cells in which both $\mathrm{HC}$ and LC rearrangements have been knocked-in.

Construction of higher numbers bnAb lineage (and other protective HIV Ab) KI mouse models can also be further aided by technologies that increase gene-targeting efficiency, and thus expedite genetic manipulations in ES cells, such as the relatively nascent "genomeediting"-based Cas9/CRISPR technology (Ran et al., 2013). This methodology allows for considerably more rapid and efficient gene targeted replacement than conventional (homologous recombination-based) gene targeting. Since many/most of the new KI mouse models the HIV field will use as immunization platforms will likely be made via similar (if not identical) Ig locus-targeting strategies (in order to allow direct head-to-head comparisons of immunogen efficacy and bnAb class/Env target feasibility to be made) they will differ only in bnAb VDJ exon specificities, or even more specifically in some cases, amongst a related series variant $\mathrm{Abs}$ (for example, progressively matured intermediate $\mathrm{Ab}$ members of a given bnAb lineage), at only a single key mutation (representing a potential selection bottleneck in bnAb development). Thus, to replace specific Ig locus $\mathrm{V}$ (D) or J segments (or $\mathrm{V}(\mathrm{D}) \mathrm{J}$ exons), i.e. essentially perform Ig mini-gene or rearrangement "swapping", ES cells with such highly similar targetable sequences can be modified using Cas9/CRISP target sites. Another process that could be used for the same purpose would be to perform such site-specific targeted replacement via use of a recombinase-based cassette exchange system (Turan et al., 2013).

Thus, with the combined application of RDBC and CRISPR for generating bnAb precursor KI models, it will now be feasible to accelerate the production bnAb KI models (from years to months, and by a degree of magnitude or even les in terms of cost), thus making it more feasible to analyze bnAb development across multiple lineages, targeting multiple Env regions other than the CD4bs. Furthermore, by using the combination of these two technologies, it will not only enable study of multiple individual bnAb lineages, but even an array of related variants, for example in addition to UCAs and matured bnAbs of key lineages, selected intermediate members (at key developmental branch points), thus making it possible to study critical maturation bottlenecks unique to particular lineages. This may be critical, since extensive SHM can either remove or introduce self-reactive residues (Haynes, Verkoczy and Kelsoe, 2014; Mietzner et al., 2008; Sabouri et al., 2014a; Shlomchik et al., 1990; Tiller et al., 2007; Verkoczy et al., 2013), and matured bnAbs can have less tolerizing self-reactivity than their precursors (Zhang et al., 2016), or potentially more, as could be the case in the $\mathrm{CH} 103$ lineage, based on reported in vitro poly/autoreactivity profiles (Liao et al., 2013b). Thus, critical lineage-specific selection bottlenecks can occur at distinct stages of maturation, and the ability to rapidly produce, and have in hand, KI models expressing intermediates will allow testing of lineage-specific vaccine regimens for their ability overcome unique bottlenecks, at an unparalleled level of resolution. 


\subsection{Further bridging the gap between practicality and translatability of Ig KI models}

\subsubsection{Conventional "Germline-reverted" and UCA precursor bnAb KI models-}

As noted earlier in Section 2, Ig KI models, due to their unique ability to balance

physiological relevance and practicality, have various advantages over other, non-genetically manipulated experimental animal models for the purpose of evaluating humoral responses to HIV vaccines, and more specifically, in understanding specific limitations and requirements for bnAb induction. In this section, we will overview various types of newer humanized Ig models, either unpublished or at the planning phases (Fig 8), in relation to those already published, with respect to their potential to further bridge this gap, and thus convert such systems from being strictly lead-generating platforms to more translational, or potentially even bona fide pre-clinical, models for testing HIV vaccine candidates.

In identifying gaps in physiological relevance still remain such models, one must ponder which critical questions remain for the field, that cannot be satisfactorily be answered by existing versions of bnAb KI models. An example of such a question is what the most effective stimulating antigens are to initiate development of bnAb lineages? A major consideration in properly designing such studies, for their being relevant to more general vaccination approaches, will be for candidate immunogens, to reproducibly bind precursor naïve B-cells bearing bona fide "gl (germline)-derived" rearrangements i.e. direct products of $\mathrm{V}(\mathrm{D}) \mathrm{J}$ recombination originating from unmutated human variable region exons (of any particular bnAb of interest originates from), that are present in the repertoire of most healthy individuals. Thus, of all human Ig KI models potentially available to test this question (Fig 8), only those capable of generating such validated "unmutated" gl-derived rearrangements, can be considered physiologically relevant. In particular, of the gl-reverted models discussed in this review, those like the VRC01gH, gl-3CN60, and gl-PGT121 models, have been made akin to conventional Ig KI models previously made in the B-cell tolerance field, i.e. with pre-rearranged V(D)Js. However, these particular models specifically use gl V segments but either retain mature HCDR3s (Dosenovic et al., 2015; Jardine et al., 2015) or "least mutated" members of a structurally-inferred (rather than time-of-infection) lineages (Escolano et al., 2016)(Fig 8; Models "1A"). Thus, it is impossible from such starting rearrangement inferences to know if certain key mutations have already been introduced that would bypass purifying selection bottlenecks during affinity maturation, nor is it possible to ascertain if clones expressing such rearrangements could be made during affinity maturation. Thus, while possibly informative as proof-of-concept for developing boosting regimen concepts and/or priming potential early intermediates of maturation pathways (assuming vaccine-induced pathways would reproducibly form similar partially-matured HCDR3 junctions) they can't reliably inform true priming phases of full maturation pathways.

In certain instances, such as when the bona fide unmutated common ancestor (UCA) of a given $\mathrm{bnAb}$ lineage has been deciphered, and/or a bnAb lineage has the potential to be reproducibly driven in multiple individuals, then using conventional KI models, in which such bnAbs" UCA V(D)J rearrangements have been knocked-in (Fig 8; Models "1B") may be warranted, since these can predict (or at least approximate) starting rearrangements of lineages with reasonable certainty. In particular, if complete virus and $\mathrm{Ab}$ co-evolutionary pathways from which a given bnAb develops has been elucidated via comprehensive 
longitudinal (time-of-infection) sampling, including a higher-probability inference of its UCA, then the "B-cell lineage immunogen design" vaccine strategy (Haynes et al., 2012b) noted in the previous section, to recapitulate, (and potentially short-circuit) maturation seen during infection, can be rigorously tested in such models. As an example, bnAb KI mice bearing the $\mathrm{CH} 103$ lineage UCA rearrangements would hold particular promise for evaluating this type of sequential vaccine approach, since the CH103 lineage's coevolutionary trajectory has been extensively sampled (Liao et al., 2013b), and includes molecular elucidation of time-of-infection samples acquired near to, or at, the transmittedfounder virus and UCA interaction step occurring in acute infection. Furthermore, the viral evolution of this lineage in the $\mathrm{CH} 505$ patient has recently been found to be faithfully recapitulated in a CH505 re-designed SHIV in macaques (Li et al., 2016), suggesting its coevolved bnAb lineage maturation trajectories may not only be reproducible across various individuals but potentially primate species.

\subsubsection{KI models expressing unrearranged minigenes from individual bnAbs-}

Unfortunately, the use of conventional KI models expressing pre-rearranged V(D)J exons are likely not to be ideal for studying vaccine-mediated maturation pathways of most currentlyidentified bnAbs, in at least two instances. First, for many (if not most) current bnAbs identified, time-of-infection samples are either not available or limited, thus restricting information regarding their molecular evolution, resulting in moderate to lower probability inferences. Thus, KI models that could address this issue, particularly for bnAbs with typical length HCDR3s, such as many of the "CD4 mimic" class of CD4bs ${ }^{+}$bnAbs, would be those engineered like the Alt lab's $\mathrm{V}_{\mathrm{H}} 1-2 \mathrm{KI}$ model (Tian et al., 2016) (Fig 8; Model "2A"), but in addition to expressing an unrearranged $\mathrm{V}_{\mathrm{H}}$, will also express the given bnAb's human $\mathrm{J}_{\mathrm{H}}$ segments (Fig 8; Model "2B"), to further ensure the relevant repertoire of V(D)J rearrangements is formed, since these mini-genes are considerably less diverse and homologous between mouse and man, than are the array of potential D segments capable of forming typical length HCDR3s (Ichihara et al., 1989). Additionally, like in the $\mathrm{V}_{\mathrm{H}} 1-2$ model, such models will insert relevant $\mathrm{V}_{\mathrm{H}}$ segments at the $\mathrm{V}_{\mathrm{H}} 81 \mathrm{X}$ site, which owing at least in part to their proximity to $\mathrm{D}$ segments, when positioned at this site will be the most frequently utilized $\mathrm{V}_{\mathrm{H}}$ segment during $\mathrm{HC}$ rearrangement (Alt et al., 2013). Also like the VH1-2 model, this bias for rearrangement at the $\mathrm{V}_{\mathrm{H}} 81 \mathrm{X}$ site can be made even more pronounced, by deletion of the regulatory element at the intergenic region between $\mathrm{V}_{\mathrm{H}}$ and D segments, the intergenic control region 1 (IGCRI), which negatively regulates $V_{H} 81 X$ rearrangement (Guo et al., 2011). Thus, such models can express a repertoire highly enriched for bnAb lineages comprised of rearrangements made from the given knocked-in $\mathrm{V}_{\mathrm{H}}$ and $\mathrm{J}_{\mathrm{H}}$ segments. Importantly, this feature distinguishes such models from "fully" Ighumanized models expressing completely polyclonal, human V(D)J-derived repertoires, i.e. Kymab® (Lee et al., 2014; Sok et al., 2016) or Regeneron VelocImmune® mice (Macdonald et al., 2014; Murphy et al., 2014) (Fig 8; Models “5”), which rarely express such bnAb rearrangements, and thus are likely to make (at least initial phases of) iterative vaccination studies aimed at inducing development of specific bnAb lineages, highly impractical.

A second instance of where conventional KI models expressing pre-rearranged V(D)J exons, including those expressing bona fide UCAs (regardless of time-of-infection information 
available) are not ideal to test prime-boost regimens for inducing bnAb maturation pathways, is for bnAbs with exceptionally long HCDR3 regions. It is estimated that $\sim 2 / 3$ of all bnAbs isolated thus far have long HCDR3s ( $\geq 20 \mathrm{aa}$ ), and those targeting the V3 or V1/V2 in particular have exceptionally long (often > 25 aa) HCDR3. Thus, for any in these latter classes especially, even for which maturation trajectories have been deciphered by longitudinal sampling, it may still be challenging to correctly deduce their UCA rearrangements, since attributing nucleotides originating from SHM rather than $\mathrm{N}$-addition at V(D)J junctions becomes increasingly difficult, the longer the HCDR (Kepler, 2013). In such cases, the current " 2 nd generation" Ig bnAb KI hypothetical model we just described, expressing unrearranged $\mathrm{V}_{\mathrm{H}}$ and $\mathrm{J}_{\mathrm{H}}$ (Fig 8; Models "2B") would also fall short, but could be modified to deal with this issue, by the further alterations to accommodate the longer human D regions (relative to those in WT mouse IgH loci), required to produce elongated HCDR3s (Briney, Willis and Crowe, 2012). Such additional modifications could involve either removing the entire murine $\mathrm{D}$ cluster as has been done before by loxP site-mediated deletion (Nitschke et al., 2001; Schelonka et al., 2005), and replacing it with a relevant long human D segment (Fig 8; Models “3”) such as D3-3, a minigene used by many long HCDR3containing bnAbs (Briney, Willis and Crowe, 2012; Willis et al., 2016), or replacing the murine $\mathrm{J}_{\mathrm{H}}$ cluster with a human $\mathrm{DJ}_{\mathrm{H}}$ containing a long $\mathrm{DJ} \mathrm{J}_{\mathrm{H}}$ junction.

\subsubsection{Limited repertoire models and other systems to increase polyclonality} and bnAb response diversity-Longer-term, models that could provide some degree of polyclonality and also generate multiple bnAb specificities, would obviously further balance the aspects of practicality with and physiological relevance for studying development of protective HIV/bnAb responses. One such model in the planning phases is the LGR;

"Limited Germline Repertoire" HIV Ab/bnAb KI model, which would preferentially express rearrangements derived from a limited set of V, D, and J segments, common to bnAbs with long HCDR3s (Fig 8; Model “4”). This latter model, representing a "hybrid” KI model between fully Ig humanized KI mice (Fig 8; Models "5") and the $2^{\text {nd }}$ generation (unrearranged) individual bnAb KI models (Fig 8; Models “3”) would be intended to provide the best balance of convenience and relevance, and thus the greatest degree of translatability (Table 2), by retaining advantages of both generating a more diverse polyclonal system, yet still allowing a restricted number of specific bnAb-specific rearrangements to predominate, relative to the much larger set of non-bnAb specificities. One major advantage with such a model is that testing of combined "bnAb lineage-targeting strategies", or more universal immunogen/vaccine approaches would be possible to evaluate at higher resolution, for their relative abilities to induce multiple bnAbs. Assuming more than one bnAb class/Env target is in fact tractable to elicit, the concept of achieving more modest breadth with such approaches, as apart of a "cumulative breath" strategy, could thus be more directly tested, and thus more rapidly translated to other pre-clinical (or even directly into clinical) trials. Shorter-term, one existing model that could have the potential to serve as such a "hybrid model", at least in some instances, could be a rat "human $\operatorname{IgH} / \mathrm{k} / \lambda$ mini-locus" transgenic model (Ma et al., 2013; Osborn et al., 2013), which is similar to the "fully Ig humanized" Kymab/Regeneron models (Fig 8; Models "5"), except that it expresses a more restricted set of human $\mathrm{V}_{\mathrm{H}}$ families, but many that are utilized by long HCDR3 bnAbs, giving it the potential to provide more convenient bnAb-specific readouts 
than the fully Ig humanized mouse platforms. However, because this model does not have the relevant bnAb genes positioned proximally, nor in the presence of the IgCR1 deletion, as the LGR model would be proposed to, nor is not clear from published reports how frequently various $\mathrm{V}_{\mathrm{H}}$ family rearrangements are found in this model's repertoire, it would need validation in the setting of HIV vaccination studies.

Other, more immediate ways to increase polyclonality of bnAb KI models for immunization studies would be adoptive transfers of either donor splenic $\mathrm{KI}^{+} \mathrm{B}$-cells into uMT recipient mice (Kitamura et al., 1991) or donor KI bone marrow cells into irradiated F1 allotype WT recipient mice, in order to generate mixed BM chimeras. In such models, the critical point is that frequencies of bnAb precursors and their repertoire can be manipulated: while the starting point will be homozygous mice to ensure bnAb precursor enrichment, degree of clonal competition can controlled by adoptive co-transfers with varying ratios of donor wildtype and/or KI B-cells. Additionally, depending on the particular experimental system and/or design used for reconstitution, two (or potentially more) $\mathrm{HIV} \mathrm{Ab/bnAb} \mathrm{precursors} \mathrm{can} \mathrm{be}$ evaluated head-to-head, in a setting where they are at limiting amounts, yet can be conveniently tracked by flow-based demarcations, either using the combination of CD45 allotypic and/or with appropriate fluorometric markers, the latter which can be achieved by deriving donor precursor KI+ B-cells from bnAb UCA models crossed to eGFP or eCFP transgenic mice. One instance where this type of in vivo system could be particularly informative is to test responses of candidate protective Ab lineages with overlapping, yet distinct Env epitopes to common immunogens. As a specific example (Fig 9), the activation and maturation of donor KI B-cell precursors from two models under development (Verkoczy and Haynes, unpublished), either eGFP+ clones expressing the UCA of CH01, a V2/glycan-specific bnAb (Bonsignori et al., 2011), or CFP+ clones expressing the UCA of CH58, a V2-specific ADCC-mediating, but minimally-neutralizing (tier1) candidate protective Ab from the RV144 trial (Liao et al., 2013a), could be evaluated in such chimera

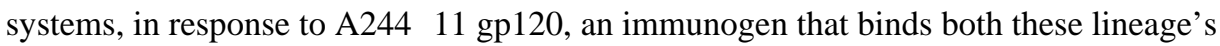
UCAs at relatively high affinity (Alam et al., 2013b). Importantly, while similar competition studies between precursors of $\mathrm{CD} 4 \mathrm{bs}^{+}$bnAb \& neutralizing Abs have been reported using transformed B-cell lines (McGuire et al., 2014), and thus may be a useful starting point for studying clonal competition dynamics in the context of priming specifically, i.e. evaluating relative BCR engagement/activation of such clones, only similar in vivo studies like the ones noted above can examine GC selection and affinity maturation in such competing lineages. Another instance where such in vivo mixed chimera systems could be useful immunization platforms are studies to understand the impact of two co-operating lineages on each others' development, in the setting of immunization, for example, co-adoptively transferring B-cells from KI mice expressing the UCA rearrangements of the $\mathrm{CH} 103$ and $\mathrm{CH} 235$ co-operating lineages (Bonsignori et al., 2016; Gao et al., 2014; Liao et al., 2013b), both KI models which are also under construction (Alt and Tian, Verkoczy and Haynes, unpublished).

Longer-term, other approaches that could also further increase translatability of such models, especially "hybrid" humanized bnAb/HIV Ab platforms like the proposed LGR strain (Fig 8; Model"4") are ones that provide increased relevance for testing vaccine strategies in pre-clinical and/or clinical settings. For example, one key issue that may impact studying T-dependent $\mathrm{Ab}$ responses in highly-inbred mouse strains, regardless of the degree 
to which models on such backgrounds have their Ig loci optimally humanized, is MHCdependence of CD4 T-cell responses. Precedent for the issue of response variability among inbred strains exists for T-dependent anti-HIV Ab responses, at least for certain bnAb targets like the MPER (Zhang et al., 2014). In this regard, backcrossing relevant bnAb KI strains like the LGR model not just into F1 backgrounds, but speed-crossing and/or re-engineering their ES cells by Cas9/CRISPR into the Collaborative Cross (CC-RIX) mouse background (Aylor et al., 2011; Churchill et al., 2004; Phillippi et al., 2014), a strain specifically outbred to mimic human population diversity, would thus be highly beneficial for the more general purpose of conferring increased class II haplotype diversity. Another example of where a further layer of translatability could potentially be added in LGR models or any such "hybrid" humanized Ig KI models having more limited (practical yet still physiologically represented) bnAb repertoires, would be to breed them to so-called humanCD4/humanCCR5 HIV-1 'infectable" transgenic models (Gruell et al., 2013; Seay et al., 2013). Such manipulations could in theory convert bnAb precursor KI repertoire platforms into direct goto humanized vaccination/challenge protection models, which if successfully validated, would not only provide the benefit of significantly reducing costs relative to existing preclinical studies (i.e. using NHP models), but may also bring to the table various other builtin benefits, such as allowing more convenient and robust determinations of any potential Abmediated protection correlates.

\subsection{Other gene-targeting manipulations beneficial for basic bnAb B-cell research \& HIV vaccine testing}

Finally, in addition to the advent of higher-throughput techniques to produce KI models, other novel approaches that may further facilitate study of bnAb development will include those in which stage-specific expression is manipulated. An instance where this could be particularly useful to study responses of bnAb $\mathrm{b}^{+} \mathrm{B}$-cells that normally are not permitted by host tolerance to progress into mature B-cell compartments, a potentially significant technical hurdle, given the large number of $2^{\text {nd }}$ generation bnAbs that also exhibit features predicted to predispose them to near-complete deletion in bone marrow, for example bnAbs like VRC26, whose unusually long HCDR3s (Doria-Rose et al., 2014) would normally subject B-cells expressing them to negative selection during early B-cell development (Meffre et al., 2001). One specific example of such an approach would be employing a CreloxP inversional recombination system under the control of a B-cell stage-specific marker's promoter such as CD21 (Maruyama, Lam and Rajewsky, 2000), to conditionally express a "passenger" bnAb specificity in the periphery, analogous to strategies previously used to modify B-cell specificities in memory B-cells (Kraus et al., 2004).

Another area where further refinements to bnAb KI models could open the door to a wider range of experimental possibilities are those providing increased flexibility for studying various mechanistic aspects of priming, maturation, and clonal competition for a various bnAb lineages. With respect to perhaps the single most critical issue, the ability to drive adequate bnAb affinity maturation, such refinements would provide greater opportunities for obtaining a deeper knowledge of whether high SHM levels are inherent to and/or generally required (or perhaps in some cases even detrimental) for bnAb development, and if so, whether inducing such high levels, or unusual, insertion/deletion events associated with such 
high SHM rates, and also key for function of many bnAbs (Kepler et al., 2014), are feasible for vaccines to elicit. In this regard, one example of bnAb KI models that could be generated to facilitate such questions are those co-expressing passenger KI alleles, which should allow a more robust ability to dissect the intrinsic mutability component of bnAb responses (Yeap et al., 2015).

Finally, one other major category of modified bnAb KI models that would benefit basic HIV B-cell research are those bred to other genetically modified $\mathrm{KI} / \mathrm{KO}$ lines with either altered SHM and/or GC B-cell selection, survival, or homing. One particularly attractive, existing candidate to which bnAb KI lines could be cross-bred, are Rev3 $3_{\mathrm{L} \rightarrow \mathrm{F}}$ mice, a KI line expressing a dominantly-active form of polymerase- $\zeta$ (Daly et al., 2012), a key molecular component of the SHM machinery (Diaz et al., 2001; Zan et al., 2001). In this KI strain, SHM rates are significantly accelerated, and thus SHM levels during immunization-guided bnAb lineage development in compound "bnAb + rev3 KI" lines would be expected to approach those typically seen during bnAb development during chronic infection. Additionally, Rev $3_{\mathrm{L}-\mathrm{F}} \mathrm{KI}$-enhanced SHM impacts all base pairs (Daly et al., 2012), in contrast to mice overexpressing activation induced deaminase (AID), the B-cell-specific (predominantly GC-restricted) enzyme required for SHM, that targets G:C bases (Okazaki et al., 2003). This is critical, since affinity-enhancing mutations are required at A:T base pairs in bnAb KI models \& for protective HIV Ab responses in general. Finally, a non-mutually exclusive set of "compound" bnAb KI lines (in addition to bnAb x rev3 KI lines) that could be generated to study if enhanced GC reaction dynamics and affinity maturation potentiates development of neutralizing breadth for various bnAb lineages, are those enhancing survival of B-cells entering, or already in, GCs. Examples of such strains would be bnAb precursor/UCA KI models cross-bred to mice overexpressing either the pro-survival gene Bcl-2, specifically in the B-cell lineage, i.e. Eu-Bcl-2 transgenic mice (Smith et al., 2000; Strasser et al., 1991) or the related protein Bcl-xL (Fang et al., 1996; Fang et al., 1998; Takahashi et al., 1999), or alternatively, to mice that are deficient in bcl2 family/pathwayassociated, pro-apoptotic genes such as Bim (Bouillet et al., 1999; Fischer et al., 2007; Oliver et al., 2006).

\section{Perspectives}

Since 2009, the HIV-1 bnAb field has undergone an emphatic revival, with immense progress made on several fronts, including: a) comprehensive longitudinal sampling of multiple cohorts of HIV-1 infected subjects and elucidation of their bnAb lineage evolutionary pathways, $\mathrm{b}$ ) the isolation and extensive molecular/structural characterization of numerous potent/broad monoclonal bnAbs from these infected subjects, c) development of novel series of immunogens and vaccine concepts that have been selected and/or engineered based on high resolution descriptions of bnAbs' paratopes, epitopes, and maturation pathways, all the result of the multiple technical advances in patient-based profiling, Ab cloning, and structural and protein modeling, and d) the generation of new animal models (as extensively discussed in this review), namely, various humanized Ig humanized KI systems and most notably, bnAb KI mice, which have been particularly powerful in obtaining a more fundamental understanding of the fate of bnAb lineages before and during immunization, have now also emerged as robust lead translational models for 
vaccine studies. The availability of novel immunogens, more powerful adjuvants, and the emergence of clinically validated immune checkpoint and/or small molecule inhibitors, combined with a better immunological understanding of roadblocks and approaches with how to overcome them, are collectively providing an unprecedented environment for basic HIV vaccine testing.

Despite this impressive level of progress, much still remains to be done towards achieving vaccine-guided bnAb induction. By all standard measures of vaccinology, we are still in the infancy of testing those critical HIV immunogens and regimens required to elicit rapid and robust bnAb responses (likely required to block HIV-1 transmission). The assertion that we are still at the "training wheels" phase of this process is perhaps truer now that ever, as it is clear, despite our best efforts to design immunogens bearing highly antigenic vaccine epitopes, that such reagents can still only elicit immunogenically-suboptimal protective $\mathrm{Ab}$ responses. Although selection of the correct Env combination and sequence of Envs, as well as their optimal form, will likely be critical for optimal bnAb induction, even the most arguably "bnAb" specific Env conformations, i.e. closed (stabilized), near-native trimeric Envs, and Env immunogens designed to target bnAbs precursors at more than adequate affinity to activate them, when used as prime components in sequential boosts, thus far do activate and guide AM in bnAb KI models (unlike repeated immunization with regular native recombinant Envs), but nevertheless still only induce development that falls short of breadth development. Furthermore, such Env immunogens, thus far, are not nearly as selective as had been hoped for, since they still predominantly induce "off-target" responses in more physiologically relevant settings, such as KI models with increased Ab repertoire diversity. In the lone case (thus far) where some breadth can develop, this is driven from a synthetically derived precursor bnAb, and thus remains to be shown if (and what) portion of a comparable, complete evolutionary pathway in humans this maturation trajectory represents. Thus, it will be important to formally evaluate if further iterations of regimens with near-native Env trimers (currently being optimized to more avidly and specifically engage bnAb precursors) alone, can drive "full" bnAb maturation pathways, i.e. from bona fide bnAb UCA to mature bnAb. However, data both from more comprehensive in vitro host protein screening methods, and from emerging bnAb UCA KI models, suggest that most bnAbs will be impeded from progressing to full breadth/potency by immune tolerance checkpoints, at some point in their maturation. These studies, and those in patient cohorts thus indicate that in addition to "correct" selection of immunogens, a suitable environment, namely, one that can promote $\mathrm{Tfh} / \mathrm{GC}$ interactions relevant to prolonging bnAb development, will also have to be recapitulated by incorporating suitable adjuvants or other transient modulators into vaccine regimens, as discussed throughout this review.

Thus, the field of HIV vaccine development is at an interesting and critical crossroads. On one side, there is strong impetus to build on results of the promising RV144 trial, including efforts to increase durability of candidate protective $\mathrm{Ab}$ responses to vaccines having nonneutralizing activities. In this regard, results from key follow-up trials are set to be reported starting late 2017, and analyses of these studies should thus help further inform this area. At the same time, the bnAb field is now more poised than ever to immerse itself into the HIV vaccine arena with unprecedented fervor, now given the virtual treasure-trove of information regarding bnAb ontogeny and structure, more powerful animal models, higher throughput 
(and resolution) technologies to probe the $\mathrm{Ab}$ repertoire, and more rational vaccine concepts, based on the combination of iterative screening, patient-based design, and reverseengineering immunogen design approaches. There is little doubt an impressive toolbox is thus now available to fully probe the immune system before and during vaccination, at a level of resolution impossible even just a few years ago. Overall, there is therefore a high degree of optimism that developing an effective preventative HIV vaccine, which includes a robust bnAb-based component, once considered an unattainable "holy grail", will come to fruition, sooner rather than later.

Finally, I point out that this review has focused exclusively on use of humanized Ig KI models for bnAb-based HIV vaccine testing, due to the wealth of information and immunogens now available for targeting these types of responses, and also because all "single $\mathrm{Ab}$ " versions of such models, thus far, have only knocked in bnAb specificities. However, such models could be equally beneficial for other HIV vaccine research, for example efforts to iteratively test formulations that seek to build on optimizing other (nonneutralizing) candidate protective responses, like those elicited in the initial RV144 trial results, especially ones aimed at optimizing durability of such responses. More generally, such models could also be employed as an approach to improve therapeutic HIV Abs (including bnAbs), via immunization-mediated AM of existing mAbs used clinically. Furthermore, and beyond the HIV vaccine field altogether, analogous bnAb KI models could also have tremendously benefit for studies seeking to better understand the regulation and induction of protective $\mathrm{Ab}$ responses to other highly variable viral infections, such as hepatitis $\mathrm{C}$ or influenza, both, which like HIV-1, require bnAb responses and are problematic vaccine targets.

\section{Acknowledgments}

Supported by NIH/NIAID grants R01AI087202 and R01AI118571, and by the Center for HIV/AIDS Vaccine Immunology and Immunogen Discovery (CHAVI-ID; grant 5UMI-AI100645 from the NIH/NIAID Division of AIDS). I thank Kevin Saunders and Howard Bomze for providing Figure 6 and Table 1, respectively. Finally, I am grateful to Bart Haynes and Garnett Kelsoe for fruitful scientific discussions regarding numerous topics covered in this review, over the past several years. There are no conflicts of interest to declare.

\section{References}

Alam SM, Aussedat B, Vohra Y, Meyerhoff RR, Cale EM, Walcowitz WE, Radakovich NA, Anasti K, Armand L, Parks R, Sutherland L, Scearce RM, Joyce MG, Pancera M, Druz A, Georgiev IS, von Holle T, Eaton A, Fox CB, Reed SG, Louder MK, Bailer RT, Morris L, Abdool-Karim SS, Cohen M, Liao HX, Montefiori DC, Park PK, Fernandez-Tejada A, Wiehe K, Santra S, Kepler TB, Saunders KO, Sodroski J, Kwong PD, Mascola JR, Bonsignori M, Moody MA, Danishefsky SJ, Haynes BF. Mimicry of an HIV Broadly Neutralizing Antibody Epitope with a Synthetic Glycopeptide. Sci Transl Med. 2017

Alam SM, Dennison SM, Aussedat B, Vohra Y, Park PK, Fernandez-Tejada A, Stewart S, Jaeger FH, Anasti K, Blinn JH, Kepler TB, Bonsignori M, Liao HX, Sodroski JG, Danishefsky SJ, Haynes BF. Recognition of synthetic glycopeptides by HIV-1 broadly neutralizing antibodies and their unmutated ancestors. Proc Natl Acad Sci U S A. 2013a; 110:18214. [PubMed: 24145434]

Alam SM, Liao HX, Dennison SM, Jaeger F, Parks R, Anasti K, Foulger A, Donathan M, Lucas J, Verkoczy L, Nicely N, Tomaras GD, Kelsoe G, Chen B, Kepler TB, Haynes BF. Differential reactivity of germ line allelic variants of a broadly neutralizing HIV-1 antibody to a gp41 fusion intermediate conformation. J Virol. 2011; 85:11725. [PubMed: 21917975] 
Alam SM, Liao HX, Tomaras GD, Bonsignori M, Tsao CY, Hwang KK, Chen H, Lloyd KE, Bowman C, Sutherland L, Jeffries TL Jr, Kozink DM, Stewart S, Anasti K, Jaeger FH, Parks R, Yates NL, Overman RG, Sinangil F, Berman PW, Pitisuttithum P, Kaewkungwal J, Nitayaphan S, Karasavva N, Rerks-Ngarm S, Kim JH, Michael NL, Zolla-Pazner S, Santra S, Letvin NL, Harrison SC, Haynes BF. Antigenicity and immunogenicity of RV144 vaccine AIDSVAX clade E envelope immunogen is enhanced by a gp120 N-terminal deletion. J Virol. 2013b; 87:1554. [PubMed: 23175357]

Alam SM, Morelli M, Dennison SM, Liao HX, Zhang R, Xia SM, Rits-Volloch S, Sun L, Harrison SC, Haynes BF, Chen B. Role of HIV membrane in neutralization by two broadly neutralizing antibodies. Proceedings of the National Academy of Sciences of the United States of America. 2009; 106:20234. [PubMed: 19906992]

Allegrucci M, Young-Cooper GO, Alexander CB, Newman BA, Mage RG. Preferrential rearrangement in normal rabbits of the $3^{\prime} \mathrm{VHa}$ allotype gene that is deleted in Alicia mutants; somatic hypermutation/conversion may play a major role in generating the heterogeneity of rabbit heavy chain variable region sequences. Eur J Immunol. 1991; 21:411. [PubMed: 1999224]

Alt FW, Zhang Y, Meng FL, Guo C, Schwer B. Mechanisms of programmed DNA lesions and genomic instability in the immune system. Cell. 2013; 152:417. [PubMed: 23374339]

Amin RH, Schlissel MS. Foxo1 directly regulates the transcription of recombination-activating genes during B cell development. Nat Immunol. 2008; 9:613. [PubMed: 18469817]

Andrabi R, Voss JE, Liang CH, Briney B, McCoy LE, Wu CY, Wong CH, Poignard P, Burton DR. Identification of Common Features in Prototype Broadly Neutralizing Antibodies to HIV Envelope V2 Apex to Facilitate Vaccine Design. Immunity. 2015; 43:959. [PubMed: 26588781]

Aplin BD, Keech CL, de Kauwe AL, Gordon TP, Cavill D, McCluskey J. Tolerance through indifference: autoreactive B cells to the nuclear antigen La show no evidence of tolerance in a transgenic model. J Immunol. 2003; 171:5890. [PubMed: 14634099]

Appelmelk BJ, Simoons-Smit I, Negrini R, Moran AP, Aspinall GO, Forte JG, De Vries T, Quan H, Verboom T, Maaskant JJ, Ghiara P, Kuipers EJ, Bloemena E, Tadema TM, Townsend RR, Tyagarajan K, Crothers JM Jr, Monteiro MA, Savio A, De Graaff J. Potential role of molecular mimicry between Helicobacter pylori lipopolysaccharide and host Lewis blood group antigens in autoimmunity. Infect Immun. 1996; 64:2031. [PubMed: 8675304]

Arnaout R, Lee W, Cahill P, Honan T, Sparrow T, Weiand M, Nusbaum C, Rajewsky K, Koralov SB. High-resolution description of antibody heavy-chain repertoires in humans. PLoS One. 2011; 6:e22365. [PubMed: 21829618]

Aylor DL, Valdar W, Foulds-Mathes W, Buus RJ, Verdugo RA, Baric RS, Ferris MT, Frelinger JA, Heise M, Frieman MB, Gralinski LE, Bell TA, Didion JD, Hua K, Nehrenberg DL, Powell CL, Steigerwalt J, Xie Y, Kelada SN, Collins FS, Yang IV, Schwartz DA, Branstetter LA, Chesler EJ, Miller DR, Spence J, Liu EY, McMillan L, Sarkar A, Wang J, Wang W, Zhang Q, Broman KW, Korstanje R, Durrant C, Mott R, Iraqi FA, Pomp D, Threadgill D, de Villena FP, Churchill GA. Genetic analysis of complex traits in the emerging Collaborative Cross. Genome Res. 2011; 21:1213. [PubMed: 21406540]

Balazs AB, Chen J, Hong CM, Rao DS, Yang L, Baltimore D. Antibody-based protection against HIV infection by vectored immunoprophylaxis. Nature. 2012; 481:81.

Barthel HR, Wallace DJ. False-positive human immunodeficiency virus testing in patients with lupus erythematosus. Semin Arthritis Rheum. 1993; 23:1. [PubMed: 8235661]

Batista FD, Neuberger MS. Affinity dependence of the B cell response to antigen: a threshold, a ceiling, and the importance of off-rate. Immunity. 1998; 8:751. [PubMed: 9655489]

Belkaid Y, Tarbell K. Regulatory T cells in the control of host-microorganism interactions (*). Annu Rev Immunol. 2009; 27:551. [PubMed: 19302048]

Benhamou D, Labi V, Novak R, Dai I, Shafir-Alon S, Weiss A, Gaujoux R, Arnold R, Shen-Orr SS, Rajewsky K, Melamed D. A c-Myc/miR17-92/Pten Axis Controls PI3K-Mediated Positive and Negative Selection in B Cell Development and Reconstitutes CD19 Deficiency. Cell Rep. 2016; 16:419. [PubMed: 27346348]

Benschop RJ, Brandl E, Chan AC, Cambier JC. Unique signaling properties of B cell antigen receptor in mature and immature B cells: implications for tolerance and activation. J Immunol. 2001; 167:4172. [PubMed: 11591737] 
Benson MJ, Erickson LD, Gleeson MW, Noelle RJ. Affinity of antigen encounter and other early Bcell signals determine B-cell fate. Curr Opin Immunol. 2007; 19:275. [PubMed: 17433651]

Bhiman JN, Anthony C, Doria-Rose NA, Karimanzira O, Schramm CA, Khoza T, Kitchin D, Botha G, Gorman J, Garrett NJ, Abdool Karim SS, Shapiro L, Williamson C, Kwong PD, Mascola JR, Morris L, Moore PL. Viral variants that initiate and drive maturation of V1V2-directed HIV-1 broadly neutralizing antibodies. Nat Med. 2015; 21:1332. [PubMed: 26457756]

Bonsignori M, Alam SM, Liao HX, Verkoczy L, Tomaras GD, Haynes BF, Moody MA. HIV-1 antibodies from infection and vaccination: insights for guiding vaccine design. Trends Microbiol. 2012a; 20:532. [PubMed: 22981828]

Bonsignori M, Hwang KK, Chen X, Tsao CY, Morris L, Gray E, Marshall DJ, Crump JA, Kapiga SH, Sam NE, Sinangil F, Pancera M, Yongping Y, Zhang B, Zhu J, Kwong PD, O'Dell S, Mascola JR, Wu L, Nabel GJ, Phogat S, Seaman MS, Whitesides JF, Moody MA, Kelsoe G, Yang X, Sodroski J, Shaw GM, Montefiori DC, Kepler TB, Tomaras GD, Alam SM, Liao HX, Haynes BF. Analysis of a clonal lineage of HIV-1 envelope V2/V3 conformational epitope-specific broadly neutralizing antibodies and their inferred unmutated common ancestors. Journal of virology. 2011; 85:9998. [PubMed: 21795340]

Bonsignori M, Kreider EF, Fera D, Meyerhoff RR, Bradley T, Wiehe K, Alam SM, Aussedat B, Walcowitz WE, Hwang K-K, Saunders KO, Zhang R, Gladden MA, Monroe A, Kumar A, Xia SM, Cooper M, Louder MK, McKee K, Bailer RT, Pier BW, Jette CA, Kelsoe G, Williams WB, Morris L, Kappes J, Wagh K, Kamanga G, Cohen MS, Hraber P, Montefiori DC, Trama AM, Liao HX, Kepler TB, Moody MA, Gao F, Danishefsky SJ, Mascola JR, Shaw GM, Hahn BH, Harrison SC, Korber B, Haynes BF. Staged induction of HIV-1 glycan-dependent broadly neutralizing antibodies. Sci Transl Med. 2017

Bonsignori M, Montefiori DC, Wu X, Chen X, Hwang KK, Tsao CY, Kozink DM, Parks RJ, Tomaras GD, Crump JA, Kapiga SH, Sam NE, Kwong PD, Kepler TB, Liao HX, Mascola JR, Haynes BF. Two distinct broadly neutralizing antibody specificities of different clonal lineages in a single HIV-1-infected donor: implications for vaccine design. J Virol. 2012b; 86:4688. [PubMed: 22301150]

Bonsignori M, Wiehe K, Grimm SK, Lynch R, Yang G, Kozink DM, Perrin F, Cooper AJ, Hwang KK, Chen X, Liu M, McKee K, Parks RJ, Eudailey J, Wang M, Clowse M, Criscione-Schreiber LG, Moody MA, Ackerman ME, Boyd SD, Gao F, Kelsoe G, Verkoczy L, Tomaras GD, Liao HX, Kepler TB, Montefiori DC, Mascola JR, Haynes BF. An autoreactive antibody from an SLE/HIV-1 individual broadly neutralizes HIV-1. The Journal of clinical investigation. 2014; 124:1835. [PubMed: 24614107]

Bonsignori M, Zhou T, Sheng Z, Chen L, Gao F, Joyce MG, Ozorowski G, Chuang GY, Schramm CA, Wiehe K, Alam SM, Bradley T, Gladden MA, Hwang KK, Iyengar S, Kumar A, Lu X, Luo K, Mangiapani MC, Parks RJ, Song H, Acharya P, Bailer RT, Cao A, Druz A, Georgiev IS, Kwon YD, Louder MK, Zhang B, Zheng A, Hill BJ, Kong R, Soto C, Mullikin JC, Douek DC, Montefiori DC, Moody MA, Shaw GM, Hahn BH, Kelsoe G, Hraber PT, Korber BT, Boyd SD, Fire AZ, Kepler TB, Shapiro L, Ward AB, Mascola JR, Liao HX, Kwong PD, Haynes BF. Maturation Pathway from Germline to Broad HIV-1 Neutralizer of a CD4-Mimic Antibody. Cell. 2016; 165:449. [PubMed: 26949186]

Bouillet P, Metcalf D, Huang DC, Tarlinton DM, Kay TW, Kontgen F, Adams JM, Strasser A. Proapoptotic Bcl-2 relative Bim required for certain apoptotic responses, leukocyte homeostasis, and to preclude autoimmunity. Science. 1999; 286:1735. [PubMed: 10576740]

Bowes T, Wagner ER, Boffey J, Nicholl D, Cochrane L, Benboubetra M, Conner J, Furukawa K, Furukawa K, Willison HJ. Tolerance to self gangliosides is the major factor restricting the antibody response to lipopolysaccharide core oligosaccharides in Campylobacter jejuni strains associated with Guillain-Barre syndrome. Infect Immun. 2002; 70:5008. [PubMed: 12183547]

Bradley T, Yang G, Ilkayeva O, Holl TM, Zhang R, Zhang J, Santra S, Fox CB, Reed SG, Parks R, Bowman CM, Bouton-Verville H, Sutherland LL, Scearce RM, Vandergrift N, Kepler TB, Moody MA, Liao HX, Alam SM, McLendon R, Everitt JI, Newgard CB, Verkoczy L, Kelsoe G, Haynes BF. HIV-1 Envelope Mimicry of Host Enzyme Kynureninase Does Not Disrupt Tryptophan Metabolism. J Immunol. 2016; 197:4663. [PubMed: 27849170] 
Braun U, Rajewsky K, Pelanda R. Different sensitivity to receptor editing of B cells from mice hemizygous or homozygous for targeted Ig transgenes. Proc Natl Acad Sci U S A. 2000; 97:7429. [PubMed: 10829061]

Briney B, Sok D, Jardine J, Kulp DW, Skog P, Menis S, Jacak R, Kalyuzhniy O, de Val N, Sesterhenn F, Le KM, Ramos A, Jones M, Saye-Francisco KL, Blane TR, Spencer S, Georgeson E, Hu X, Ozorowski G, Adachi Y, Kubitz M, Sarkar A, Wilson IA, Ward AB, Nemazee D, Burton DR, Schief WR. Tailored Immunogens direct affinity maruration toward HIV neutralizing antibodies. Cell. 2016; 166:1459. [PubMed: 27610570]

Briney BS, Willis JR, Crowe JE Jr. Human peripheral blood antibodies with long HCDR3s are established primarily at original recombination using a limited subset of germline genes. PLoS One. 2012; 7:e36750. [PubMed: 22590602]

Burton DR, Ahmed R, Barouch DH, Butera ST, Crotty S, Godzik A, Kaufmann DE, McElrath MJ, Nussenzweig MC, Pulendran B, Scanlan CN, Schief WR, Silvestri G, Streeck H, Walker BD, Walker LM, Ward AB, Wilson IA, Wyatt R. A Blueprint for HIV Vaccine Discovery. Cell Host Microbe. 2012; 12:396. [PubMed: 23084910]

Burton DR, Hangartner L. Broadly Neutralizing Antibodies to HIV and Their Role in Vaccine Design. Annu Rev Immunol. 2016; 34:635. [PubMed: 27168247]

Burton DR, Mascola JR. Antibody responses to envelope glycoproteins in HIV-1 infection. Nat Immunol. 2015; 16:571. [PubMed: 25988889]

Calza L, Manfredi R, Colangeli V, D’ Antuono A, Passarini B, Chiodo F. Systemic and discoid lupus erythematosus in HIV-infected patients treated with highly active antiretroviral therapy. Int J STD AIDS. 2003; 14:356. [PubMed: 12803945]

Cambier JC, Gauld SB, Merrell KT, Vilen BJ. B-cell anergy: from transgenic models to naturally occurring anergic B cells? Nat Rev Immunol. 2007; 7:633. [PubMed: 17641666]

Chambers CA, Kuhns MS, Egen JG, Allison JP. CTLA-4-mediated inhibition in regulation of T cell responses: mechanisms and manipulation in tumor immunotherapy. Annu Rev Immunol. 2001; 19:565. [PubMed: 11244047]

Chaturvedi A, Dorward D, Pierce SK. The B cell receptor governs the subcellular location of Toll-like receptor 9 leading to hyperresponses to DNA-containing antigens. Immunity. 2008; 28:799. [PubMed: 18513998]

Chen C, Nagy Z, Prak EL, Weigert M. Immunoglobulin heavy chain gene replacement: a mechanism of receptor editing. Immunity. 1995a; 3:747. [PubMed: 8777720]

Chen C, Nagy Z, Radic MZ, Hardy RR, Huszar D, Camper SA, Weigert M. The site and stage of antiDNA B-cell deletion. Nature. 1995b; 373:252. [PubMed: 7816141]

Chen J. Analysis of gene function in lymphocytes by RAG-2-deficient blastocyst complementation. Adv Immunol. 1996; 62:31. [PubMed: 8781266]

Chen J, Lansford R, Stewart V, Young F, Alt FW. RAG-2-deficient blastocyst complementation: an assay of gene function in lymphocyte development. Proc Natl Acad Sci U S A. 1993; 90:4528. [PubMed: 8506294]

Chen J, Shinkai Y, Young F, Alt FW. Probing immune functions in RAG-deficient mice. Curr Opin Immunol. 1994; 6:313. [PubMed: 8011215]

Chen Y, Zhang J, Bouton-Verville H, Newman A, Xia SM, Liao HX, Montefiori DC, Moody MA, Armand L, Hwang KK, Lockwood B, Dennison SM, Alam SM, Haynes BB, Verkoczy L. Lipid components in MPER-based immunization regimens are critical for inducing broadly neutralizing antibody responses in 2F5 and 4E10 knock-in Mice. AIDS Research and Human Retroviruses. 2013a; 29:20. [PubMed: 22891715]

Chen Y, Zhang J, Hwang KK, Bouton-Verville H, Xia SM, Newman A, Ouyang YB, Haynes BF, Verkoczy L. Common tolerance mechanisms, but distinct cross-reactivities associated with gp41 and lipids, limit production of HIV-1 broad neutralizing antibodies 2F5 and 4E10. J Immunol. 2013b; 191:1260. [PubMed: 23825311]

Chow KT, Timblin GA, McWhirter SM, Schlissel MS. MK5 activates Rag transcription via Foxo1 in developing B cells. J Exp Med. 2013; 210:1621. [PubMed: 23878308]

Churchill GA, Airey DC, Allayee H, Angel JM, Attie AD, Beatty J, Beavis WD, Belknap JK, Bennett B, Berrettini W, Bleich A, Bogue M, Broman KW, Buck KJ, Buckler E, Burmeister M, Chesler EJ, 
Cheverud JM, Clapcote S, Cook MN, Cox RD, Crabbe JC, Crusio WE, Darvasi A, Deschepper CF, Doerge RW, Farber CR, Forejt J, Gaile D, Garlow SJ, Geiger H, Gershenfeld H, Gordon T, Gu J, Gu W, de Haan G, Hayes NL, Heller C, Himmelbauer H, Hitzemann R, Hunter K, Hsu HC, Iraqi FA, Ivandic B, Jacob HJ, Jansen RC, Jepsen KJ, Johnson DK, Johnson TE, Kempermann G, Kendziorski C, Kotb M, Kooy RF, Llamas B, Lammert F, Lassalle JM, Lowenstein PR, Lu L, Lusis A, Manly KF, Marcucio R, Matthews D, Medrano JF, Miller DR, Mittleman G, Mock BA, Mogil JS, Montagutelli X, Morahan G, Morris DG, Mott R, Nadeau JH, Nagase H, Nowakowski RS, O’Hara BF, Osadchuk AV, Page GP, Paigen B, Paigen K, Palmer AA, Pan HJ, Peltonen-Palotie L, Peirce J, Pomp D, Pravenec M, Prows DR, Qi Z, Reeves RH, Roder J, Rosen GD, Schadt EE, Schalkwyk LC, Seltzer Z, Shimomura K, Shou S, Sillanpaa MJ, Siracusa LD, Snoeck HW, Spearow JL, Svenson K. The Collaborative Cross, a community resource for the genetic analysis of complex traits. Nat Genet. 2004; 36:1133. [PubMed: 15514660]

Clark BE, Auyeung K, Fregolino E, Parrilli M, Lanzetta R, De Castro C, Pantophlet R. A bacterial lipooligosaccharide that naturally mimics the epitope of the HIV-neutralizing antibody $2 \mathrm{G} 12$ as a template for vaccine design. Chem Biol. 2012; 19:254. [PubMed: 22365608]

Coffre M, Benhamou D, Riess D, Blumenberg L, Snetkova V, Hines MJ, Chakraborty T, Bajwa S, Jensen K, Chong MM, Getu L, Silverman GJ, Blelloch R, Littman DR, Calado D, Melamed D, Skok JA, Rajewsky K, Koralov SB. miRNAs Are Essential for the Regulation of the PI3K/AKT/ FOXO Pathway and Receptor Editing during B Cell Maturation. Cell Rep. 2016; 17:2271. [PubMed: 27880903]

Cooke MP, Heath AW, Shokat KM, Zeng Y, Finkelman FD, Linsley PS, Howard M, Goodnow CC. Immunoglobulin signal transduction guides the specificity of B cell-T cell interactions and is blocked in tolerant self-reactive B cells. J Exp Med. 1994; 179:425. [PubMed: 8294858]

Corti D, Lanzavecchia A. Broadly neutralizing antiviral antibodies. Annu Rev Immunol. 2013; 31:705. [PubMed: 23330954]

Cusick MF, Libbey JE, Fujinami RS. Molecular mimicry as a mechanism of autoimmune disease. Clin Rev Allergy Immunol. 2012; 42:102. [PubMed: 22095454]

Cyster JG, Hartley SB, Goodnow CC. Competition for follicular niches excludes self-reactive cells from the recirculating B-cell repertoire. Nature. 1994; 371:389. [PubMed: 7522305]

Dal Porto JM, Haberman AM, Kelsoe G, Shlomchik MJ. Very low affinity B cells form germinal centers, become memory B cells, and participate in secondary immune responses when higher affinity competition is reduced. The Journal of experimental medicine. 2002; 195:1215. [PubMed: 11994427]

Dal Porto JM, Haberman AM, Shlomchik MJ, Kelsoe G. Antigen drives very low affinity B cells to become plasmacytes and enter germinal centers. Journal of immunology. 1998; 161:5373.

Daly J, Bebenek K, Watt DL, Richter K, Jiang C, Zhao ML, Ray M, McGregor WG, Kunkel TA, Diaz M. Altered Ig hypermutation pattern and frequency in complementary mouse models of DNA polymerase zeta activity. J Immunol. 2012; 188:5528. [PubMed: 22547703]

de Taeye SW, Moore JP, Sanders RW. HIV-1 Envelope Trimer Design and Immunization Strategies To Induce Broadly Neutralizing Antibodies. Trends Immunol. 2016; 37:221. [PubMed: 26869204]

de Taeye SW, Ozorowski G, Torrents de la Pena A, Guttman M, Julien JP, van den Kerkhof TL, Burger JA, Pritchard LK, Pugach P, Yasmeen A, Crampton J, Hu J, Bontjer I, Torres JL, Arendt H, DeStefano J, Koff WC, Schuitemaker H, Eggink D, Berkhout B, Dean H, LaBranche C, Crotty S, Crispin M, Montefiori DC, Klasse PJ, Lee KK, Moore JP, Wilson IA, Ward AB, Sanders RW. Immunogenicity of Stabilized HIV-1 Envelope Trimers with Reduced Exposure of Nonneutralizing Epitopes. Cell. 2015; 163:1702. [PubMed: 26687358]

DeKosky BJ, Kojima T, Rodin A, Charab W, Ippolito GC, Ellington AD, Georgiou G. In-depth determination and analysis of the human paired heavy- and light-chain antibody repertoire. Nat Med. 2015; 21:86. [PubMed: 25501908]

Diaz M, Klinman NR. Relative roles of somatic and Darwinian evolution in shaping the antibody response. Immunol Res. 2000; 21:89. [PubMed: 10852106]

Diaz M, Verkoczy LK, Flajnik MF, Klinman NR. Decreased frequency of somatic hypermutation and impaired affinity maturation but intact germinal center formation in mice expressing antisense RNA to DNA polymerase zeta. J Immunol. 2001; 167:327. [PubMed: 11418667] 
Doores KJ, Kong L, Krumm SA, Le KM, Sok D, Laserson U, Garces F, Poignard P, Wilson IA, Burton DR. Two classes of broadly neutralizing antibodies within a single lineage directed to the highmannose patch of HIV envelope. J Virol. 2015; 89:1105. [PubMed: 25378488]

Doria-Rose NA, Louder MK, Yang Z, O’Dell S, Nason M, Schmidt SD, McKee K, Seaman MS, Bailer RT, Mascola JR. HIV-1 neutralization coverage is improved by combining monoclonal antibodies that target independent epitopes. J Virol. 2012; 86:3393. [PubMed: 22258252]

Doria-Rose NA, Schramm CA, Gorman J, Moore PL, Bhiman JN, Dekosky BJ, Ernandes MJ, Georgiev IS, Kim HJ, Pancera M, Staupe RP, Altae-Tran HR, Bailer RT, Crooks ET, Cupo A, Druz A, Garrett NJ, Hoi KH, Kong R, Louder MK, Longo NS, McKee K, Nonyane M, O’Dell S, Roark RS, Rudicell RS, Schmidt SD, Sheward DJ, Soto C, Wibmer CK, Yang Y, Zhang Z, Mullikin JC, Binley JM, Sanders RW, Wilson IA, Moore JP, Ward AB, Georgiou G, Williamson C, Abdool Karim SS, Morris L, Kwong PD, Shapiro L, Mascola JR. Nisc Comparative S. Developmental pathway for potent V1V2-directed HIV-neutralizing antibodies. Nature. 2014; 509:55. [PubMed: 24590074]

Dormitzer PR, Grandi G, Rappuoli R. Structural vaccinology starts to deliver. Nat Rev Microbiol. 2012; 10:807. [PubMed: 23154260]

Dosenovic P, Soldemo M, Scholz JL, O’Dell S, Grasset EK, Pelletier N, Karlsson MC, Mascola JR, Wyatt RT, Cancro MP, Karlsson Hedestam GB. BLyS-mediated modulation of naive B cell subsets impacts HIV Env-induced antibody responses. J Immunol. 2012; 188:6018. [PubMed: 22561155]

Dosenovic P, von Boehmer L, Escolano A, Jardine J, Freund NT, Gitlin AD, McGuire AT, Kulp DW, Oliveira T, Scharf L, Pietzsch J, Gray MD, Cupo A, van Gils MJ, Yao KH, Liu C, Gazumyan A, Seaman MS, Bjorkman PJ, Sanders RW, Moore JP, Stamatatos L, Schief WR, Nussenzweig MC. Immunization for HIV-1 Broadly Neutralizing Antibodies in Human Ig Knockin Mice. Cell. 2015; 161:1505. [PubMed: 26091035]

Doyle-Cooper C, Hudson KE, Cooper AB, Ota T, Skog P, Dawson PE, Zwick MB, Schief WR, Burton DR, Nemazee D. Immune tolerance negatively regulates B cells in knock-in mice expressing broadly neutralizing HIV antibody 4E10. J Immunol. 2013; 191:3186. [PubMed: 23940276]

Eda Y, Takizawa M, Murakami T, Maeda H, Kimachi K, Yonemura H, Koyanagi S, Shiosaki K, Higuchi H, Makizumi K, Nakashima T, Osatomi K, Tokiyoshi S, Matsushita S, Yamamoto N, Honda M. Sequential immunization with V3 peptides from primary human immunodeficiency virus type 1 produces cross-neutralizing antibodies against primary isolates with a matching narrow-neutralization sequence motif. J Virol. 2006; 80:5552. [PubMed: 16699036]

Eisen HN, Siskind GW. VARIATIONS IN AFFINITIES OF ANTIBODIES DURING THE IMMUNE RESPONSE. Biochemistry. 1964; 3:996. [PubMed: 14214095]

Erikson J, Radic MZ, Camper SA, Hardy RR, Carmack C, Weigert M. Expression of anti-DNA immunoglobulin transgenes in non-autoimmune mice. Nature. 1991; 349:331. [PubMed: 1898987]

Escolano A, Steichen JM, Dosenovic P, Kulp DW, Golijanin J, Sok D, Freund NT, Gitlin AD, Oliveira T, Araki T, Lowe S, Chen ST, Heinemann J, Yao KH, Georgeson E, Saye-Francisco KF, Gazumyan A, Burton DR, Schief WR, Nussenzweig MC. Sequential immunization elicits broadly neutralizing anti-HIV antibodies in Ig knock-in mice. Cell. 2016; 166:1445. [PubMed: 27610569]

Excler JL, Ake J, Robb ML, Kim JH, Plotkin SA. Nonneutralizing functional antibodies: a new "old" paradigm for HIV vaccines. Clin Vaccine Immunol. 2014; 21:1023. [PubMed: 24920599]

Fang W, Mueller DL, Pennell CA, Rivard JJ, Li YS, Hardy RR, Schlissel MS, Behrens TW. Frequent aberrant immunoglobulin gene rearrangements in pro-B cells revealed by a bcl-xL transgene. Immunity. 1996; 4:291. [PubMed: 8624819]

Fang W, Weintraub BC, Dunlap B, Garside P, Pape KA, Jenkins MK, Goodnow CC, Mueller DL, Behrens TW. Self-reactive B lymphocytes overexpressing Bcl-xL escape negative selection and are tolerized by clonal anergy and receptor editing. Immunity. 1998; 9:35. [PubMed: 9697834]

Finton KA, Larimore K, Larman HB, Friend D, Correnti C, Rupert PB, Elledge SJ, Greenberg PD, Strong RK. Autoreactivity and exceptional CDR plasticity (but not unusual polyspecificity) hinder elicitation of the anti-HIV antibody 4E10. PLoS Pathog. 2013; 9:e1003639. [PubMed: 24086134]

Fischer SF, Bouillet P, O’Donnell K, Light A, Tarlinton DM, Strasser A. Proapoptotic BH3-only protein Bim is essential for developmentally programmed death of germinal center-derived memory B cells and antibody-forming cells. Blood. 2007; 110:3978. [PubMed: 17720882] 
Gao F, Bonsignori M, Liao HX, Kumar A, Xia SM, Lu X, Cai F, Hwang KK, Song H, Zhou T, Lynch RM, Alam SM, Moody MA, Ferrari G, Berrong M, Kelsoe G, Shaw GM, Hahn BH, Montefiori DC, Kamanga G, Cohen MS, Hraber P, Kwong PD, Korber BT, Mascola JR, Kepler TB, Haynes BF. Cooperation of B Cell Lineages in Induction of HIV-1-Broadly Neutralizing Antibodies. Cell. 2014; 158:481. [PubMed: 25065977]

Garces F, Lee JH, de Val N, de la Pena AT, Kong L, Puchades C, Hua Y, Stanfield RL, Burton DR, Moore JP, Sanders RW, Ward AB, Wilson IA. Affinity Maturation of a Potent Family of HIV Antibodies Is Primarily Focused on Accommodating or Avoiding Glycans. Immunity. 2015; 43:1053. [PubMed: 26682982]

Garces F, Sok D, Kong L, McBride R, Kim HJ, Saye-Francisco KF, Julien JP, Hua Y, Cupo A, Moore JP, Paulson JC, Ward AB, Burton DR, Wilson IA. Structural evolution of glycan recognition by a family of potent HIV antibodies. Cell. 2014; 159:69. [PubMed: 25259921]

Gautam R, Nishimura Y, Pegu A, Nason MC, Klein F, Gazumyan A, Golijanin J, Buckler-White A, Sadjadpour R, Wang K, Mankoff Z, Schmidt SD, Lifson JD, Mascola JR, Nussenzweig MC, Martin MA. A single injection of anti-HIV-1 antibodies protects against repeated SHIV challenges. Nature. 2016; 533:105. [PubMed: 27120156]

Gay D, Saunders T, Camper S, Weigert M. Receptor editing: an approach by autoreactive B cells to escape tolerance. J Exp Med. 1993; 177:999. [PubMed: 8459227]

Georgiev IS, Rudicell RS, Saunders KO, Shi W, Kirys T, McKee K, O’Dell S, Chuang GY, Yang ZY, Ofek G, Connors M, Mascola JR, Nabel GJ, Kwong PD. Antibodies VRC01 and 10E8 neutralize HIV-1 with high breadth and potency even with Ig-framework regions substantially reverted to germline. J Immunol. 2014; 192:1100. [PubMed: 24391217]

Goepfert PA, Elizaga ML, Sato A, Qin L, Cardinali M, Hay CM, Hural J, DeRosa SC, DeFawe OD, Tomaras GD, Montefiori DC, Xu Y, Lai L, Kalams SA, Baden LR, Frey SE, Blattner WA, Wyatt LS, Moss B, Robinson HL. Phase 1 safety and immunogenicity testing of DNA and recombinant modified vaccinia Ankara vaccines expressing HIV-1 virus-like particles. J Infect Dis. 2011; 203:610. [PubMed: 21282192]

Gonzalez-Martin A, Adams BD, Lai M, Shepherd J, Salvador-Bernaldez M, Salvador JM, Lu J, Nemazee D, Xiao C. The microRNA miR-148a functions as a critical regulator of B cell tolerance and autoimmunity. Nat Immunol. 2016; 17:433. [PubMed: 26901150]

Goodnow CC. Transgenic mice and analysis of B-cell tolerance. Annu Rev Immunol. 1992; 10:489. [PubMed: 1590994]

Goodnow CC. Multistep pathogenesis of autoimmune disease. Cell. 2007; 130:25. [PubMed: 17632054]

Goodnow CC, Brink R, Adams E. Breakdown of self-tolerance in anergic B lymphocytes. Nature. 1991; 352:532. [PubMed: 1830923]

Goodnow CC, Crosbie J, Adelstein S, Lavoie TB, Smith-Gill SJ, Brink RA, Pritchard-Briscoe H, Wotherspoon JS, Loblay RH, Raphael K, et al. Altered immunoglobulin expression and functional silencing of self-reactive B lymphocytes in transgenic mice. Nature. 1988; 334:676. [PubMed: 3261841]

Goodnow CC, Crosbie J, Jorgensen H, Brink RA, Basten A. Induction of self-tolerance in mature peripheral B lymphocytes. Nature. 1989; 342:385. [PubMed: 2586609]

Goodnow CC, Vinuesa CG, Randall KL, Mackay F, Brink R. Control systems and decision making for antibody production. Nat Immunol. 2010; 11:681. [PubMed: 20644574]

Gorman J, Soto C, Yang MM, Davenport TM, Guttman M, Bailer RT, Chambers M, Chuang GY, DeKosky BJ, Doria-Rose NA, Druz A, Ernandes MJ, Georgiev IS, Jarosinski MC, Joyce MG, Lemmin TM, Leung S, Louder MK, McDaniel JR, Narpala S, Pancera M, Stuckey J, Wu X, Yang Y, Zhang B, Zhou T, Mullikin JC, Baxa U, Georgiou G, McDermott AB, Bonsignori M, Haynes BF, Moore PL, Morris L, Lee KK, Shapiro L, Mascola JR, Kwong PD. Structures of HIV-1 Env V1V2 with broadly neutralizing antibodies reveal commonalities that enable vaccine design. Nat Struct Mol Biol. 2016; 23:81. [PubMed: 26689967]

Gray ES, Madiga MC, Hermanus T, Moore PL, Wibmer CK, Tumba NL, Werner L, Mlisana K, Sibeko S, Williamson C, Abdool Karim SS, Morris L. The neutralization breadth of HIV-1 develops incrementally over four years and is associated with CD4+ T cell decline and high viral load during acute infection. Journal of virology. 2011; 85:4828. [PubMed: 21389135] 
Gruell H, Bournazos S, Ravetch JV, Ploss A, Nussenzweig MC, Pietzsch J. Antibody and antiretroviral preexposure prophylaxis prevent cervicovaginal HIV-1 infection in a transgenic mouse model. $\mathrm{J}$ Virol. 2013; 87:8535. [PubMed: 23720722]

Guo C, Yoon HS, Franklin A, Jain S, Ebert A, Cheng HL, Hansen E, Despo O, Bossen C, Vettermann C, Bates JG, Richards N, Myers D, Patel H, Gallagher M, Schlissel MS, Murre C, Busslinger M, Giallourakis CC, Alt FW. CTCF-binding elements mediate control of V(D)J recombination. Nature. 2011; 477:424. [PubMed: 21909113]

Gupta S, Clark ES, Termini JM, Boucher J, Kanagavelu S, LeBranche CC, Abraham S, Montefiori DC, Khan WN, Stone GW. DNA vaccine molecular adjuvants SP-D-BAFF and SP-D-APRIL enhance anti-gp120 immune response and increase HIV-1 neutralizing antibody titers. J Virol. 2015; 89:4158. [PubMed: 25631080]

Han S, Zheng B, Dal Porto J, Kelsoe G. In situ studies of the primary immune response to (4hydroxy-3-nitrophenyl)acetyl. IV. Affinity-dependent, antigen-driven B cell apoptosis in germinal centers as a mechanism for maintaining self-tolerance. J Exp Med. 1995; 182:1635. [PubMed: 7500008]

Hansen SG, Piatak M Jr, Ventura AB, Hughes CM, Gilbride RM, Ford JC, Oswald K, Shoemaker R, Li Y, Lewis MS, Gilliam AN, Xu G, Whizin N, Burwitz BJ, Planer SL, Turner JM, Legasse AW, Axthelm MK, Nelson JA, Fruh K, Sacha JB, Estes JD, Keele BF, Edlefsen PT, Lifson JD, Picker LJ. Immune clearance of highly pathogenic SIV infection. Nature. 2013a; 502:100. [PubMed: 24025770]

Hansen SG, Sacha JB, Hughes CM, Ford JC, Burwitz BJ, Scholz I, Gilbride RM, Lewis MS, Gilliam AN, Ventura AB, Malouli D, Xu G, Richards R, Whizin N, Reed JS, Hammond KB, Fischer M, Turner JM, Legasse AW, Axthelm MK, Edlefsen PT, Nelson JA, Lifson JD, Fruh K, Picker LJ. Cytomegalovirus vectors violate CD8+ T cell epitope recognition paradigms. Science. 2013b; 340:1237874. [PubMed: 23704576]

Hardy RR. B-1 B cells: development, selection, natural autoantibody and leukemia. Curr Opin Immunol. 2006; 18:547. [PubMed: 16879952]

Hartley SB, Crosbie J, Brink R, Kantor AB, Basten A, Goodnow CC. Elimination from peripheral lymphoid tissues of self-reactive B lymphocytes recognizing membrane-bound antigens. Nature. 1991; 353:765. [PubMed: 1944535]

Hayakawa K, Asano M, Shinton SA, Gui M, Wen LJ, Dashoff J, Hardy RR. Positive selection of antithy-1 autoreactive B-1 cells and natural serum autoantibody production independent from bone marrow B cell development. J Exp Med. 2003; 197:87. [PubMed: 12515816]

Haynes BF. New approaches to HIV vaccine development. Curr Opin Immunol. 2015; 35:39. [PubMed: 26056742]

Haynes BF, Fleming J, St Clair EW, Katinger H, Stiegler G, Kunert R, Robinson J, Scearce RM, Plonk K, Staats HF, Ortel TL, Liao HX, Alam SM. Cardiolipin polyspecific autoreactivity in two broadly neutralizing HIV-1 antibodies. Science. 2005a; 308:1906. [PubMed: 15860590]

Haynes BF, Gilbert PB, McElrath MJ, Zolla-Pazner S, Tomaras GD, Alam SM, Evans DT, Montefiori DC, Karnasuta C, Sutthent R, Liao HX, DeVico AL, Lewis GK, Williams C, Pinter A, Fong Y, Janes H, DeCamp A, Huang Y, Rao M, Billings E, Karasavvas N, Robb ML, Ngauy V, de Souza MS, Paris R, Ferrari G, Bailer RT, Soderberg KA, Andrews C, Berman PW, Frahm N, De Rosa SC, Alpert MD, Yates NL, Shen X, Koup RA, Pitisuttithum P, Kaewkungwal J, Nitayaphan S, Rerks-Ngarm S, Michael NL, Kim JH. Immune-correlates analysis of an HIV-1 vaccine efficacy trial. The New England journal of medicine. 2012a; 366:1275. [PubMed: 22475592]

Haynes BF, Kelsoe G, Harrison SC, Kepler TB. B-cell-lineage immunogen design in vaccine development with HIV-1 as a case study. Nature biotechnology. 2012b; 30:423.

Haynes BF, Moody MA, Alam M, Bonsignori M, Verkoczy L, Ferrari G, Gao F, Tomaras GD, Liao HX, Kelsoe G. Progress in HIV-1 vaccine development. J Allergy Clin Immunol. 2014; 134:3. [PubMed: 25117798]

Haynes BF, Moody MA, Liao HX, Verkoczy L, Tomaras GD. B cell responses to HIV-1 infection and vaccination: pathways to preventing infection. Trends Mol Med. 2011; 17:108. [PubMed: 21112250]

Haynes BF, Moody MA, Verkoczy L, Kelsoe G, Alam SM. Antibody polyspecificity and neutralization of HIV-1: a hypothesis. Hum Antibodies. 2005b; 14:59. [PubMed: 16720975] 
Haynes BF, Shaw GM, Korber B, Kelsoe G, Sodroski J, Hahn BH, Borrow P, McMichael AJ. HIVHost Interactions: Implications for Vaccine Design. Cell Host Microbe. 2016; 19:292. [PubMed: 26922989]

Haynes BF, Verkoczy L. AIDS/HIV. Host controls of HIV neutralizing antibodies. Science. 2014; 344:588. [PubMed: 24812389]

Haynes BF, Verkoczy L, Kelsoe G. Redemption of autoreactive B cells. Proc Natl Acad Sci U S A. 2014; 111:9022. [PubMed: 24920593]

Hessell AJ, Poignard P, Hunter M, Hangartner L, Tehrani DM, Bleeker WK, Parren PW, Marx PA, Burton DR. Effective, low-titer antibody protection against low-dose repeated mucosal SHIV challenge in macaques. Nat Med. 2009a; 15:951. [PubMed: 19525965]

Hessell AJ, Rakasz EG, Poignard P, Hangartner L, Landucci G, Forthal DN, Koff WC, Watkins DI, Burton DR. Broadly neutralizing human anti-HIV antibody $2 \mathrm{G} 12$ is effective in protection against mucosal SHIV challenge even at low serum neutralizing titers. PLoS Pathog. 2009b; 5:e1000433. [PubMed: 19436712]

Hoot S, McGuire AT, Cohen KW, Strong RK, Hangartner L, Klein F, Diskin R, Scheid JF, Sather DN, Burton DR, Stamatatos L. Recombinant HIV envelope proteins fail to engage germline versions of anti-CD4bs bNAbs. PLoS Pathog. 2013; 9:e1003106. [PubMed: 23300456]

Hraber P, Seaman MS, Bailer RT, Mascola JR, Montefiori DC, Korber BT. Prevalence of broadly neutralizing antibody responses during chronic HIV-1 infection. Aids. 2014; 28:163. [PubMed: 24361678]

Huang J, Ofek G, Laub L, Louder MK, Doria-Rose NA, Longo NS, Imamichi H, Bailer RT, Chakrabarti B, Sharma SK, Alam SM, Wang T, Yang Y, Zhang B, Migueles SA, Wyatt R, Haynes BF, Kwong PD, Mascola JR, Connors M. Broad and potent neutralization of HIV-1 by a gp41-specific human antibody. Nature. 2012; 491:406. [PubMed: 23151583]

Huang Y, DiazGranados C, Janes H, Huang Y, deCamp AC, Metch B, Grant S, Sanchez B, Phogat S, Koutsoukos M, Kanesa-Thasan N, Bourguignon P, Collard A, Buchbinder S, Tomaras GD, McElrath J, Gray G, Kublin JG, Corey L, Gilbert PB. Selection of HIV vaccine candidates for concurrent testing in an efficacy trial. Curr Opin Virol. 2016; 17:57. [PubMed: 26827165]

Ichihara Y, Hayashida H, Miyazawa S, Kurosawa Y. Only DFL16, DSP2, and DQ52 gene families exist in mouse immunoglobulin heavy chain diversity gene loci, of which DFL16 and DSP2 originate from the same primordial DH gene. Eur J Immunol. 1989; 19:1849. [PubMed: 2583225]

Ivanov II, Schelonka RL, Zhuang Y, Gartland GL, Zemlin M, Schroeder HW Jr. Development of the expressed Ig CDR-H3 repertoire is marked by focusing of constraints in length, amino acid use, and charge that are first established in early B cell progenitors. J Immunol. 2005; 174:7773. [PubMed: 15944280]

Jardine J, Julien JP, Menis S, Ota T, Kalyuzhniy O, McGuire A, Sok D, Huang PS, MacPherson S, Jones M, Nieusma T, Mathison J, Baker D, Ward AB, Burton DR, Stamatatos L, Nemazee D, Wilson IA, Schief WR. Rational HIV immunogen design to target specific germline B cell receptors. Science. 2013; 340:711. [PubMed: 23539181]

Jardine JG, Kulp DW, Havenar-Daughton C, Sarkar A, Briney B, Sok D, Sesterhenn F, Ereno-Orbea J, Kalyuzhniy O, Deresa I, Hu X, Spencer S, Jones M, Georgeson E, Adachi Y, Kubitz M, deCamp AC, Julien JP, Wilson IA, Burton DR, Crotty S, Schief WR. HIV-1 broadly neutralizing antibody precursor B cells revealed by germline-targeting immunogen. Science. 2016a; 351:1458. [PubMed: 27013733]

Jardine JG, Ota T, Sok D, Pauthner M, Kulp DW, Kalyuzhniy O, Skog PD, Thinnes TC, Bhullar D, Briney B, Menis S, Jones M, Kubitz M, Spencer S, Adachi Y, Burton DR, Schief WR, Nemazee D. HIV-1 VACCINES. Priming a broadly neutralizing antibody response to HIV-1 using a germline-targeting immunogen. Science. 2015; 349:156. [PubMed: 26089355]

Jardine JG, Sok D, Julien JP, Briney B, Sarkar A, Liang CH, Scherer EA, Henry Dunand CJ, Adachi Y, Diwanji D, Hsueh J, Jones M, Kalyuzhniy O, Kubitz M, Spencer S, Pauthner M, Saye-Francisco KL, Sesterhenn F, Wilson PC, Galloway DM, Stanfield RL, Wilson IA, Burton DR, Schief WR. Minimally Mutated HIV-1 Broadly Neutralizing Antibodies to Guide Reductionist Vaccine Design. PLoS Pathog. 2016b; 12:e1005815. [PubMed: 27560183] 
Julien JP, Lee JH, Cupo A, Murin CD, Derking R, Hoffenberg S, Caulfield MJ, King CR, Marozsan AJ, Klasse PJ, Sanders RW, Moore JP, Wilson IA, Ward AB. Asymmetric recognition of the HIV-1 trimer by broadly neutralizing antibody PG9. Proc Natl Acad Sci U S A. 2013; 110:4351. [PubMed: 23426631]

Kain R, Exner M, Brandes R, Ziebermayr R, Cunningham D, Alderson CA, Davidovits A, Raab I, Jahn R, Ashour O, Spitzauer S, Sunder-Plassmann G, Fukuda M, Klemm P, Rees AJ, Kerjaschki D. Molecular mimicry in pauci-immune focal necrotizing glomerulonephritis. Nat Med. 2008; 14:1088. [PubMed: 18836458]

Kaye BR. Rheumatologic manifestations of infection with human immunodeficiency virus (HIV). Ann Intern Med. 1989; 111:158. [PubMed: 2662849]

Keenan RA, De Riva A, Corleis B, Hepburn L, Licence S, Winkler TH, Martensson IL. Censoring of autoreactive B cell development by the pre-B cell receptor. Science. 2008; 321:696. [PubMed: 18566249]

Keir ME, Butte MJ, Freeman GJ, Sharpe AH. PD-1 and its ligands in tolerance and immunity. Annu Rev Immunol. 2008; 26:677. [PubMed: 18173375]

Kelsoe G, Verkoczy L, Haynes BF. Immune System Regulation in the Induction of Broadly Neutralizing HIV-1 Antibodies. Vaccines (Basel). 2014; 2:1. [PubMed: 24932410]

Kepler TB. Reconstructing a B-cell clonal lineage. I. Statistical inference of unobserved ancestors. F1000Research. 2013; 2:103. [PubMed: 24555054]

Kepler TB, Liao HX, Alam SM, Bhaskarabhatla R, Zhang R, Yandava C, Stewart S, Anasti K, Kelsoe G, Parks R, Lloyd KE, Stolarchuk C, Pritchett J, Solomon E, Friberg E, Morris L, Karim SS, Cohen MS, Walter E, Moody MA, Wu X, Altae-Tran HR, Georgiev IS, Kwong PD, Boyd SD, Fire AZ, Mascola JR, Haynes BF. Immunoglobulin gene insertions and deletions in the affinity maturation of HIV-1 broadly reactive neutralizing antibodies. Cell Host Microbe. 2014; 16:304. [PubMed: 25211073]

Kepler TB, Perelson AS. Somatic hypermutation in B cells: an optimal control treatment. J Theor Biol. 1993; 164:37. [PubMed: 8264243]

Kepler TB, Perelson AS. Modeling and optimization of populations subject to time-dependent mutation. Proc Natl Acad Sci U S A. 1995; 92:8219. [PubMed: 7667271]

Kitamura D, Roes J, Kuhn R, Rajewsky K. A B cell-deficient mouse by targeted disruption of the membrane exon of the immunoglobulin mu chain gene. Nature. 1991; 350:423. [PubMed: 1901381]

Klein F, Diskin R, Scheid JF, Gaebler C, Mouquet H, Georgiev IS, Pancera M, Zhou T, Incesu RB, Fu BZ, Gnanapragasam PN, Oliveira TY, Seaman MS, Kwong PD, Bjorkman PJ, Nussenzweig MC. Somatic mutations of the immunoglobulin framework are generally required for broad and potent HIV-1 neutralization. Cell. 2013; 153:126. [PubMed: 23540694]

Klein U, Casola S, Cattoretti G, Shen Q, Lia M, Mo T, Ludwig T, Rajewsky K, Dalla-Favera R. Transcription factor IRF4 controls plasma cell differentiation and class-switch recombination. Nat Immunol. 2006; 7:773. [PubMed: 16767092]

Klinman DM, Higgins KW, Conover J. Sequential immunizations with rgp120s from independent isolates of human immunodeficiency virus type 1 induce the preferential expansion of broadly crossreactive B cells. J Exp Med. 1991; 173:881. [PubMed: 2007856]

Knight KL. Restricted VH gene usage and generation of antibody diversity in rabbit. Annu Rev Immunol. 1992; 10:593. [PubMed: 1590997]

Kong L, Ju B, Chen Y, He L, Ren L, Liu J, Hong K, Su B, Wang Z, Ozorowski G, Ji X, Hua Y, Chen Y, Deller MC, Hao Y, Feng Y, Garces F, Wilson R, Dai K, O’Dell S, McKee K, Mascola JR, Ward AB, Wyatt RT, Li Y, Wilson IA, Zhu J, Shao Y. Key gp120 Glycans Pose Roadblocks to the Rapid Development of VRC01-Class Antibodies in an HIV-1-Infected Chinese Donor. Immunity. 2016; 44:939. [PubMed: 27067056]

Kraus M, Alimzhanov MB, Rajewsky N, Rajewsky K. Survival of resting mature B lymphocytes depends on BCR signaling via the Igalpha/beta heterodimer. Cell. 2004; 117:787. [PubMed: 15186779]

Kumar KR, Mohan C. Understanding B-cell tolerance through the use of immunoglobulin transgenic models. Immunol Res. 2008; 40:208. [PubMed: 17943232] 
Kuraoka M, Snowden PB, Nojima T, Verkoczy L, Haynes BF, Kitamura D, Kelsoe G. A New Pathway for Central B cell Tolerance: Interaction of AID, MyD88, and BCR. Cell Reports. 2017 In Press.

Kuwahara K, Fujimura S, Takahashi Y, Nakagata N, Takemori T, Aizawa S, Sakaguchi N. Germinal center-associated nuclear protein contributes to affinity maturation of B cell antigen receptor in $\mathrm{T}$ cell-dependent responses. Proc Natl Acad Sci U S A. 2004; 101:1010. [PubMed: 14715907]

Kuwahara K, Tomiyasu S, Fujimura S, Nomura K, Xing Y, Nishiyama N, Ogawa M, Imajoh-Ohmi S, Izuta S, Sakaguchi N. Germinal center-associated nuclear protein (GANP) has a phosphorylationdependent DNA-primase activity that is up-regulated in germinal center regions. Proc Natl Acad Sci U S A. 2001; 98:10279. [PubMed: 11526238]

Kwon YD, Pancera M, Acharya P, Georgiev IS, Crooks ET, Gorman J, Joyce MG, Guttman M, Ma X, Narpala S, Soto C, Terry DS, Yang Y, Zhou T, Ahlsen G, Bailer RT, Chambers M, Chuang GY, Doria-Rose NA, Druz A, Hallen MA, Harned A, Kirys T, Louder MK, O’Dell S, Ofek G, Osawa K, Prabhakaran M, Sastry M, Stewart-Jones GB, Stuckey J, Thomas PV, Tittley T, Williams C, Zhang B, Zhao H, Zhou Z, Donald BR, Lee LK, Zolla-Pazner S, Baxa U, Schon A, Freire E, Shapiro L, Lee KK, Arthos J, Munro JB, Blanchard SC, Mothes W, Binley JM, McDermott AB, Mascola JR, Kwong PD. Crystal structure, conformational fixation and entry-related interactions of mature ligand-free HIV-1 Env. Nat Struct Mol Biol. 2015; 22:522. [PubMed: 26098315]

Kwong PD, Mascola JR. Human antibodies that neutralize HIV-1: identification, structures, and B cell ontogenies. Immunity. 2012; 37:412. [PubMed: 22999947]

Lai M, Gonzalez-Martin A, Cooper AB, Oda H, Jin HY, Shepherd J, He L, Zhu J, Nemazee D, Xiao C. Regulation of B-cell development and tolerance by different members of the miR-17 approximately 92 family microRNAs. Nat Commun. 2016; 7:12207. [PubMed: 27481093]

Lang J, Jackson M, Teyton L, Brunmark A, Kane K, Nemazee D. B cells are exquisitely sensitive to central tolerance and receptor editing induced by ultralow affinity, membrane-bound antigen. J Exp Med. 1996; 184:1685. [PubMed: 8920858]

Lanzavecchia A, Fruhwirth A, Perez L, Corti D. Antibody-guided vaccine design: identification of protective epitopes. Curr Opin Immunol. 2016; 41:62. [PubMed: 27343848]

Larman HB, Zhao Z, Laserson U, Li MZ, Ciccia A, Gakidis MA, Church GM, Kesari S, Leproust EM, Solimini NL, Elledge SJ. Autoantigen discovery with a synthetic human peptidome. Nat Biotechnol. 2011; 29:535. [PubMed: 21602805]

Lau CM, Broughton C, Tabor AS, Akira S, Flavell RA, Mamula MJ, Christensen SR, Shlomchik MJ, Viglianti GA, Rifkin IR, Marshak-Rothstein A. RNA-associated autoantigens activate B cells by combined B cell antigen receptor/Toll-like receptor 7 engagement. J Exp Med. 2005; 202:1171. [PubMed: 16260486]

Leadbetter EA, Rifkin IR, Hohlbaum AM, Beaudette BC, Shlomchik MJ, Marshak-Rothstein A. Chromatin-IgG complexes activate B cells by dual engagement of IgM and Toll-like receptors. Nature. 2002; 416:603. [PubMed: 11948342]

Lee CH, Melchers M, Wang H, Torrey TA, Slota R, Qi CF, Kim JY, Lugar P, Kong HJ, Farrington L, van der Zouwen B, Zhou JX, Lougaris V, Lipsky PE, Grammer AC, Morse HC 3rd. Regulation of the germinal center gene program by interferon (IFN) regulatory factor $8 /$ IFN consensus sequence-binding protein. J Exp Med. 2006; 203:63. [PubMed: 16380510]

Lee EC, Liang Q, Ali H, Bayliss L, Beasley A, Bloomfield-Gerdes T, Bonoli L, Brown R, Campbell J, Carpenter A, Chalk S, Davis A, England N, Fane-Dremucheva A, Franz B, Germaschewski V, Holmes H, Holmes S, Kirby I, Kosmac M, Legent A, Lui H, Manin A, O’Leary S, Paterson J, Sciarrillo R, Speak A, Spensberger D, Tuffery L, Waddell N, Wang W, Wells S, Wong V, Wood A, Owen MJ, Friedrich GA, Bradley A. Complete humanization of the mouse immunoglobulin loci enables efficient therapeutic antibody discovery. Nat Biotechnol. 2014; 32:356. [PubMed: 24633243]

Levesque MC, Moody MA, Hwang KK, Marshall DJ, Whitesides JF, Amos JD, Gurley TC, Allgood S, Haynes BB, Vandergrift NA, Plonk S, Parker DC, Cohen MS, Tomaras GD, Goepfert PA, Shaw GM, Schmitz JE, Eron JJ, Shaheen NJ, Hicks CB, Liao HX, Markowitz M, Kelsoe G, Margolis DM, Haynes BF. Polyclonal B cell differentiation and loss of gastrointestinal tract germinal centers in the earliest stages of HIV-1 infection. PLoS Med. 2009; 6:e1000107. [PubMed: 19582166] 
Li H, Jiang Y, Prak EL, Radic M, Weigert M. Editors and editing of anti-DNA receptors. Immunity. 2001; 15:947. [PubMed: 11754816]

Li H, Wang S, Kong R, Ding W, Lee FH, Parker Z, Kim E, Learn GH, Hahn P, Policicchio B, BroccaCofano E, Deleage C, Hao X, Chuang GY, Gorman J, Gardner M, Lewis MG, Hatziioannou T, Santra S, Apetrei C, Pandrea I, Alam SM, Liao HX, Shen X, Tomaras GD, Farzan M, Chertova E, Keele BF, Estes JD, Lifson JD, Doms RW, Montefiori DC, Haynes BF, Sodroski JG, Kwong PD, Hahn BH, Shaw GM. Envelope residue 375 substitutions in simian-human immunodeficiency viruses enhance CD4 binding and replication in rhesus macaques. Proc Natl Acad Sci U S A. 2016; 113:E3413. [PubMed: 27247400]

Li Y, Li H, Weigert M. Autoreactive B cells in the marginal zone that express dual receptors. J Exp Med. 2002; 195:181. [PubMed: 11805145]

Li Y, O’Dell S, Walker LM, Wu X, Guenaga J, Feng Y, Schmidt SD, McKee K, Louder MK, Ledgerwood JE, Graham BS, Haynes BF, Burton DR, Wyatt RT, Mascola JR. Mechanism of neutralization by the broadly neutralizing HIV-1 monoclonal antibody VRC01. J Virol. 2011; 85:8954. [PubMed: 21715490]

Liao HX, Bonsignori M, Alam SM, McLellan JS, Tomaras GD, Moody MA, Kozink DM, Hwang KK, Chen X, Tsao CY, Liu P, Lu X, Parks RJ, Montefiori DC, Ferrari G, Pollara J, Rao M, Peachman KK, Santra S, Letvin NL, Karasavvas N, Yang ZY, Dai K, Pancera M, Gorman J, Wiehe K, Nicely NI, Rerks-Ngarm S, Nitayaphan S, Kaewkungwal J, Pitisuttithum P, Tartaglia J, Sinangil F, Kim JH, Michael NL, Kepler TB, Kwong PD, Mascola JR, Nabel GJ, Pinter A, Zolla-Pazner $\mathrm{S}$, Haynes BF. Vaccine induction of antibodies against a structurally heterogeneous site of immune pressure within HIV-1 envelope protein variable regions 1 and 2. Immunity. 2013a; 38:176. [PubMed: 23313589]

Liao HX, Lynch R, Zhou T, Gao F, Alam SM, Boyd SD, Fire AZ, Roskin KM, Schramm CA, Zhang Z, Zhu J, Shapiro L, Mullikin JC, Gnanakaran S, Hraber P, Wiehe K, Kelsoe G, Yang G, Xia SM, Montefiori DC, Parks R, Lloyd KE, Scearce RM, Soderberg KA, Cohen M, Kamanga G, Louder MK, Tran LM, Chen Y, Cai F, Chen S, Moquin S, Du X, Joyce MG, Srivatsan S, Zhang B, Zheng A, Shaw GM, Hahn BH, Kepler TB, Korber BT, Kwong PD, Mascola JR, Haynes BF. Coevolution of a broadly neutralizing HIV-1 antibody and founder virus. Nature. 2013b; 496:469. [PubMed: 23552890]

Lifson JD, Piatak M Jr, Rossio JL, Bess J Jr, Chertova E, Schneider D, Kiser R, Coalter V, Poore B, Imming R, Desrosiers RC, Henderson LE, Arthur LO. Whole inactivated SIV virion vaccines with functional envelope glycoproteins: safety, immunogenicity, and activity against intrarectal challenge. J Med Primatol. 2002; 31:205. [PubMed: 12390543]

Linton PL, Decker DJ, Klinman NR. Primary antibody-forming cells and secondary B cells are generated from separate precursor cell subpopulations. Cell. 1989; 59:1049. [PubMed: 2598260]

Liu M, Yang G, Wiehe K, Nicely NI, Vandergrift NA, Rountree W, Bonsignori M, Alam SM, Gao J, Haynes BF, Kelsoe G. Polyreactivity and autoreactivity among HIV-1 antibodies. J Virol. 2015; 89:784. [PubMed: 25355869]

Llorian M, Stamataki Z, Hill S, Turner M, Martensson IL. The PI3K p110delta is required for downregulation of RAG expression in immature B cells. J Immunol. 2007; 178:1981. [PubMed: 17277100]

Locci M, Wu JE, Arumemi F, Mikulski Z, Dahlberg C, Miller AT, Crotty S. Activin A programs the differentiation of human TFH cells. Nat Immunol. 2016; 17:976. [PubMed: 27376469]

Ma B, Osborn MJ, Avis S, Ouisse LH, Menoret S, Anegon I, Buelow R, Bruggemann M. Human antibody expression in transgenic rats: comparison of chimeric IgH loci with human VH, D and JH but bearing different rat C-gene regions. J Immunol Methods. 2013; 400-401:78.

Ma BJ, Alam SM, Go EP, Lu X, Desaire H, Tomaras GD, Bowman C, Sutherland LL, Scearce RM, Santra S, Letvin NL, Kepler TB, Liao HX, Haynes BF. Envelope deglycosylation enhances antigenicity of HIV-1 gp41 epitopes for both broad neutralizing antibodies and their unmutated ancestor antibodies. PLoS Pathog. 2011; 7:e1002200. [PubMed: 21909262]

Macdonald LE, Karow M, Stevens S, Auerbach W, Poueymirou WT, Yasenchak J, Frendewey D, Valenzuela DM, Giallourakis CC, Alt FW, Yancopoulos GD, Murphy AJ. Precise and in situ genetic humanization of $6 \mathrm{Mb}$ of mouse immunoglobulin genes. Proc Natl Acad Sci U S A. 2014; 111:5147. [PubMed: 24706858] 
Malherbe DC, Doria-Rose NA, Misher L, Beckett T, Puryear WB, Schuman JT, Kraft Z, O’Malley J, Mori M, Srivastava I, Barnett S, Stamatatos L, Haigwood NL. Sequential immunization with a subtype B HIV-1 envelope quasispecies partially mimics the in vivo development of neutralizing antibodies. J Virol. 2011; 85:5262. [PubMed: 21430056]

Margine I, Krammer F, Hai R, Heaton NS, Tan GS, Andrews SA, Runstadler JA, Wilson PC, Albrecht RA, Garcia-Sastre A, Palese P. Hemagglutinin stalk-based universal vaccine constructs protect against group 2 influenza A viruses. J Virol. 2013; 87:10435. [PubMed: 23903831]

Marshak-Rothstein A, Rifkin IR. Immunologically active autoantigens: the role of toll-like receptors in the development of chronic inflammatory disease. Annu Rev Immunol. 2007; 25:419. [PubMed: 17378763]

Martin DA, Bradl H, Collins TJ, Roth E, Jack HM, Wu GE. Selection of Ig mu heavy chains by complementarity-determining region 3 length and amino acid composition. J Immunol. 2003; 171:4663. [PubMed: 14568941]

Martin F, Kearney JF. Marginal-zone B cells. Nat Rev Immunol. 2002; 2:323. [PubMed: 12033738]

Maruyama M, Lam KP, Rajewsky K. Memory B-cell persistence is independent of persisting immunizing antigen. Nature. 2000; 407:636. [PubMed: 11034213]

Mascola JR, Haynes BF. HIV-1 neutralizing antibodies: understanding nature's pathways. Immunological reviews. 2013; 254:225. [PubMed: 23772623]

McElrath MJ, Haynes BF. Induction of immunity to human immunodeficiency virus type-1 by vaccination. Immunity. 2010; 33:542. [PubMed: 21029964]

McGuire AT, Dreyer AM, Carbonetti S, Lippy A, Glenn J, Scheid JF, Mouquet H, Stamatatos L. HIV antibodies. Antigen modification regulates competition of broad and narrow neutralizing HIV antibodies. Science. 2014; 346:1380. [PubMed: 25504724]

McGuire AT, Gray MD, Dosenovic P, Gitlin AD, Freund NT, Petersen J, Correnti C, Johnsen W, Kegel R, Stuart AB, Glenn J, Seaman MS, Schief WR, Strong RK, Nussenzweig MC, Stamatatos L. Specifically modified Env immunogens activate B-cell precursors of broadly neutralizing HIV-1 antibodies in transgenic mice. Nat Commun. 2016; 7:10618. [PubMed: 26907590]

McGuire AT, Hoot S, Dreyer AM, Lippy A, Stuart A, Cohen KW, Jardine J, Menis S, Scheid JF, West AP, Schief WR, Stamatatos L. Engineering HIV envelope protein to activate germline B cell receptors of broadly neutralizing anti-CD4 binding site antibodies. J Exp Med. 2013; 210:655. [PubMed: 23530120]

McMichael AJ, Borrow P, Tomaras GD, Goonetilleke N, Haynes BF. The immune response during acute HIV-1 infection: clues for vaccine development. Nat Rev Immunol. 2010; 10:11. [PubMed: 20010788]

Meffre E, Milili M, Blanco-Betancourt C, Antunes H, Nussenzweig MC, Schiff C. Immunoglobulin heavy chain expression shapes the B cell receptor repertoire in human B cell development. J Clin Invest. 2001; 108:879. [PubMed: 11560957]

Melkus MW, Estes JD, Padgett-Thomas A, Gatlin J, Denton PW, Othieno FA, Wege AK, Haase AT, Garcia JV. Humanized mice mount specific adaptive and innate immune responses to EBV and TSST-1. Nat Med. 2006; 12:1316. [PubMed: 17057712]

Mietzner B, Tsuiji M, Scheid J, Velinzon K, Tiller T, Abraham K, Gonzalez JB, Pascual V, Stichweh D, Wardemann H, Nussenzweig MC. Autoreactive IgG memory antibodies in patients with systemic lupus erythematosus arise from nonreactive and polyreactive precursors. Proc Natl Acad Sci U S A. 2008; 105:9727. [PubMed: 18621685]

Mikell I, Sather DN, Kalams SA, Altfeld M, Alter G, Stamatatos L. Characteristics of the earliest cross-neutralizing antibody response to HIV-1. PLoS Pathog. 2011; 7:e1001251. [PubMed: 21249232]

Minegishi Y, Conley ME. Negative selection at the pre-BCR checkpoint elicited by human mu heavy chains with unusual CDR3 regions. Immunity. 2001; 14:631. [PubMed: 11371364]

Moir S, Fauci AS. Insights into B cells and HIV-specific B-cell responses in HIV-infected individuals. Immunol Rev. 2013; 254:207. [PubMed: 23772622]

Mond JJ, Vos Q, Lees A, Snapper CM. T cell independent antigens. Curr Opin Immunol. 1995; 7:349. [PubMed: 7546399] 
Moody MA, Pedroza-Pacheco I, Vandergrift NA, Chui C, Lloyd KE, Parks R, Soderberg KA, Ogbe AT, Cohen MS, Liao HX, Gao F, McMichael AJCMD, Verkoczy LGK, Huang JRSP, Connors M, Borrow P, Haynes BF. Immune perturbations in HIV-1-infected individuals who make broadly neutralizing antibodies. Science Immunology. 2016; 1:aag0851. [PubMed: 28783677]

Moore PL, Gray ES, Wibmer CK, Bhiman JN, Nonyane M, Sheward DJ, Hermanus T, Bajimaya S, Tumba NL, Abrahams MR, Lambson BE, Ranchobe N, Ping L, Ngandu N, Abdool Karim Q, Abdool Karim SS, Swanstrom RI, Seaman MS, Williamson C, Morris L. Evolution of an HIV glycan-dependent broadly neutralizing antibody epitope through immune escape. Nature medicine. 2012; 18:1688.

Moore PL, Williamson C. Approaches to the induction of HIV broadly neutralizing antibodies. Curr Opin HIV AIDS. 2016; 11:569. [PubMed: 27559709]

Moore PL, Williamson C, Morris L. Virological features associated with the development of broadly neutralizing antibodies to HIV-1. Trends Microbiol. 2015; 23:204. [PubMed: 25572881]

Mouquet H, Scharf L, Euler Z, Liu Y, Eden C, Scheid JF, Halper-Stromberg A, Gnanapragasam PN, Spencer DI, Seaman MS, Schuitemaker H, Feizi T, Nussenzweig MC, Bjorkman PJ. Complextype $\mathrm{N}$-glycan recognition by potent broadly neutralizing HIV antibodies. Proc Natl Acad Sci U S A. 2012; 109:E3268. [PubMed: 23115339]

Mouquet H, Scheid JF, Zoller MJ, Krogsgaard M, Ott RG, Shukair S, Artyomov MN, Pietzsch J, Connors M, Pereyra F, Walker BD, Ho DD, Wilson PC, Seaman MS, Eisen HN, Chakraborty AK, Hope TJ, Ravetch JV, Wardemann H, Nussenzweig MC. Polyreactivity increases the apparent affinity of anti-HIV antibodies by heteroligation. Nature. 2010; 467:591. [PubMed: 20882016]

Murakami M, Tsubata T, Okamoto M, Shimizu A, Kumagai S, Imura H, Honjo T. Antigen-induced apoptotic death of Ly-1 B cells responsible for autoimmune disease in transgenic mice. Nature. 1992; 357:77. [PubMed: 1574128]

Murphy AJ, Macdonald LE, Stevens S, Karow M, Dore AT, Pobursky K, Huang TT, Poueymirou WT, Esau L, Meola M, Mikulka W, Krueger P, Fairhurst J, Valenzuela DM, Papadopoulos N, Yancopoulos GD. Mice with megabase humanization of their immunoglobulin genes generate antibodies as efficiently as normal mice. Proc Natl Acad Sci U S A. 2014; 111:5153. [PubMed: 24706856]

Mylonakis E, Paliou M, Greenbough TC, Flaningan TP, Letvin NL, Rich JD. Report of a false-positive HIV test result and the potential use of additional tests in establishing HIV serostatus. Arch Intern Med. 2000; 160:2386. [PubMed: 10927739]

Nachbagauer R, Wohlbold TJ, Hirsh A, Hai R, Sjursen H, Palese P, Cox RJ, Krammer F. Induction of broadly reactive anti-hemagglutinin stalk antibodies by an H5N1 vaccine in humans. J Virol. 2014; 88:13260. [PubMed: 25210189]

Nemazee DA, Burki K. Clonal deletion of B lymphocytes in a transgenic mouse bearing anti-MHC class I antibody genes. Nature. 1989; 337:562. [PubMed: 2783762]

Nitschke L, Kestler J, Tallone T, Pelkonen S, Pelkonen J. Deletion of the DQ52 element within the Ig heavy chain locus leads to a selective reduction in VDJ recombination and altered D gene usage. J Immunol. 2001; 166:2540. [PubMed: 11160315]

Okamoto M, Murakami M, Shimizu A, Ozaki S, Tsubata T, Kumagai S, Honjo T. A transgenic model of autoimmune hemolytic anemia. J Exp Med. 1992; 175:71. [PubMed: 1730928]

Okazaki I, Yoshikawa K, Kinoshita K, Muramatsu M, Nagaoka H, Honjo T. Activation-induced cytidine deaminase links class switch recombination and somatic hypermutation. Ann N Y Acad Sci. 2003; 987:1. [PubMed: 12727618]

Oliver PM, Vass T, Kappler J, Marrack P. Loss of the proapoptotic protein, Bim, breaks B cell anergy. J Exp Med. 2006; 203:731. [PubMed: 16520387]

Osborn MJ, Ma B, Avis S, Binnie A, Dilley J, Yang X, Lindquist K, Menoret S, Iscache AL, Ouisse LH, Rajpal A, Anegon I, Neuberger MS, Buelow R, Bruggemann M. High-affinity IgG antibodies develop naturally in Ig-knockout rats carrying germline human IgH/Igkappa/Iglambda loci bearing the rat CH region. J Immunol. 2013; 190:1481. [PubMed: 23303672] 
Ota T, Aoki-Ota M, Tsunoda K, Simoda K, Nishikawa T, Amagai M, Koyasu S. Auto-reactive B cells against peripheral antigen, desmoglein 3, escape from tolerance mechanism. Int Immunol. 2004; 16:1487. [PubMed: 15339881]

Ota T, Doyle-Cooper C, Cooper AB, Doores KJ, Aoki-Ota M, Le K, Schief WR, Wyatt RT, Burton DR, Nemazee D. B cells from knock-in mice expressing broadly neutralizing HIV antibody b12 carry an innocuous B cell receptor responsive to HIV vaccine candidates. J Immunol. 2013; 191:3179. [PubMed: 23940273]

Ota T, Doyle-Cooper C, Cooper AB, Huber M, Falkowska E, Doores KJ, Hangartner L, Le K, Sok D, Jardine J, Lifson J, Wu X, Mascola JR, Poignard P, Binley JM, Chakrabarti BK, Schief WR, Wyatt RT, Burton DR, Nemazee D. Anti-HIV B Cell lines as candidate vaccine biosensors. J Immunol. 2012; 189:4816. [PubMed: 23066156]

Palacios R, Santos J, Valdivielso P, Marquez M. Human immunodeficiency virus infection and systemic lupus erythematosus. An unusual case and a review of the literature. Lupus. 2002; 11:60. [PubMed: 11898923]

Pancera M, Shahzad-Ul-Hussan S, Doria-Rose NA, McLellan JS, Bailer RT, Dai K, Loesgen S, Louder MK, Staupe RP, Yang Y, Zhang B, Parks R, Eudailey J, Lloyd KE, Blinn J, Alam SM, Haynes BF, Amin MN, Wang LX, Burton DR, Koff WC, Nabel GJ, Mascola JR, Bewley CA, Kwong PD. Structural basis for diverse N-glycan recognition by HIV-1-neutralizing V1-V2-directed antibody PG16. Nat Struct Mol Biol. 2013; 20:804. [PubMed: 23708607]

Pantophlet R, Burton DR. GP120: target for neutralizing HIV-1 antibodies. Annu Rev Immunol. 2006; 24:739. [PubMed: 16551265]

Patel P, Kearney JF. Immunological Outcomes of Antibody Binding to Glycans Shared between Microorganisms and Mammals. J Immunol. 2016; 197:4201. [PubMed: 27864551]

Paus D, Phan TG, Chan TD, Gardam S, Basten A, Brink R. Antigen recognition strength regulates the choice between extrafollicular plasma cell and germinal center B cell differentiation. J Exp Med. 2006; 203:1081. [PubMed: 16606676]

Pejchal R, Doores KJ, Walker LM, Khayat R, Huang PS, Wang SK, Stanfield RL, Julien JP, Ramos A, Crispin M, Depetris R, Katpally U, Marozsan A, Cupo A, Maloveste S, Liu Y, McBride R, Ito Y, Sanders RW, Ogohara C, Paulson JC, Feizi T, Scanlan CN, Wong CH, Moore JP, Olson WC, Ward AB, Poignard P, Schief WR, Burton DR, Wilson IA. A potent and broad neutralizing antibody recognizes and penetrates the HIV glycan shield. Science. 2011; 334:1097. [PubMed: 21998254]

Pejchal R, Walker LM, Stanfield RL, Phogat SK, Koff WC, Poignard P, Burton DR, Wilson IA. Structure and function of broadly reactive antibody PG16 reveal an $\mathrm{H} 3$ subdomain that mediates potent neutralization of HIV-1. Proc Natl Acad Sci U S A. 2010; 107:11483. [PubMed: 20534513]

Phan TG, Paus D, Chan TD, Turner ML, Nutt SL, Basten A, Brink R. High affinity germinal center B cells are actively selected into the plasma cell compartment. J Exp Med. 2006; 203:2419. [PubMed: 17030950]

Phillippi J, Xie Y, Miller DR, Bell TA, Zhang Z, Lenarcic AB, Aylor DL, Krovi SH, Threadgill DW, de Villena FP, Wang W, Valdar W, Frelinger JA. Using the emerging Collaborative Cross to probe the immune system. Genes Immun. 2014; 15:38. [PubMed: 24195963]

Plotkin SA. Correlates of protection induced by vaccination. Clin Vaccine Immunol. 2010; 17:1055. [PubMed: 20463105]

Pujanauski LM, Janoff EN, McCarter MD, Pelanda R, Torres RM. Mouse marginal zone B cells harbor specificities similar to human broadly neutralizing HIV antibodies. Proc Natl Acad Sci U S A. 2013; 110:1422. [PubMed: 23288906]

Pulendran B, Kannourakis G, Nouri S, Smith KG, Nossal GJ. Soluble antigen can cause enhanced apoptosis of germinal-centre B cells. Nature. 1995; 375:331. [PubMed: 7753199]

Raman VS, Lind EF, Benson MJ, Noelle RJ. Strategies for selective priming of memory B cells. Immunol Lett. 2007; 109:93. [PubMed: 17374402]

Ran FA, Hsu PD, Wright J, Agarwala V, Scott DA, Zhang F. Genome engineering using the CRISPRCas9 system. Nat Protoc. 2013; 8:2281. [PubMed: 24157548] 
Rerks-Ngarm S, Pitisuttithum P, Nitayaphan S, Kaewkungwal J, Chiu J, Paris R, Premsri N, Namwat C, de Souza M, Adams E, Benenson M, Gurunathan S, Tartaglia J, McNeil JG, Francis DP, Stablein D, Birx DL, Chunsuttiwat S, Khamboonruang C, Thongcharoen P, Robb ML, Michael NL, Kunasol P, Kim JH. Vaccination with ALVAC and AIDSVAX to prevent HIV-1 infection in Thailand. N Engl J Med. 2009; 361:2209. [PubMed: 19843557]

Richter K, Brar S, Ray M, Pisitkun P, Bolland S, Verkoczy L, Diaz M. Speckled-like pattern in the germinal center (SLIP-GC), a nuclear GTPase expressed in activation-induced deaminaseexpressing lymphomas and germinal center B cells. J Biol Chem. 2009; 284:30652. [PubMed: 19734146]

Richter K, Burch L, Chao F, Henke D, Jiang C, Daly J, Zhao ML, Kissling G, Diaz M. Altered pattern of immunoglobulin hypermutation in mice deficient in Slip-GC protein. J Biol Chem. 2012; 287:31856. [PubMed: 22833677]

Rojas M, Hulbert C, Thomas JW. Anergy and not clonal ignorance determines the fate of B cells that recognize a physiological autoantigen. J Immunol. 2001; 166:3194. [PubMed: 11207272]

Russell DM, Dembic Z, Morahan G, Miller JF, Burki K, Nemazee D. Peripheral deletion of selfreactive B cells. Nature. 1991; 354:308. [PubMed: 1956380]

Sabouri Z, Schofield P, Horikawa K, Spierings E, Kipling D, Randall KL, Langley D, Roome B, Vazquez-Lombardi R, Rouet R. Redemption of autoantibodies on anergic B cells by variableregion glycosylation and mutation away from self-reactivity. 2014a; 111:E2567.

Sabouri Z, Schofield P, Horikawa K, Spierings E, Kipling D, Randall KL, Langley D, Roome B, Vazquez-Lombardi R, Rouet R, Hermes J, Chan TD, Brink R, Dunn-Walters DK, Christ D, Goodnow CC. Redemption of autoantibodies on anergic B cells by variable-region glycosylation and mutation away from self-reactivity. Proc Natl Acad Sci U S A. 2014b; 111:E2567. [PubMed: 24821781]

Sakaguchi N, Kimura T, Matsushita S, Fujimura S, Shibata J, Araki M, Sakamoto T, Minoda C, Kuwahara K. Generation of high-affinity antibody against T cell-dependent antigen in the Ganp gene-transgenic mouse. J Immunol. 2005; 174:4485. [PubMed: 15814669]

Sanders RW, van Gils MJ, Derking R, Sok D, Ketas TJ, Burger JA, Ozorowski G, Cupo A, Simonich C, Goo L, Arendt H, Kim HJ, Lee JH, Pugach P, Williams M, Debnath G, Moldt B, van Breemen MJ, Isik G, Medina-Ramirez M, Back JW, Koff WC, Julien JP, Rakasz EG, Seaman MS, Guttman M, Lee KK, Klasse PJ, LaBranche C, Schief WR, Wilson IA, Overbaugh J, Burton DR, Ward AB, Montefiori DC, Dean H, Moore JP. HIV-1 VACCINES. HIV-1 neutralizing antibodies induced by native-like envelope trimers. Science. 2015; 349:aac4223. [PubMed: 26089353]

Scharf L, West AP, Sievers SA, Chen C, Jiang S, Gao H, Gray MD, McGuire AT, Scheid JF, Nussenzweig MC, Stamatatos L, Bjorkman PJ. Structural basis for germline antibody recognition of HIV-1 immunogens. Elife. 2016:5.

Scheid JF, Mouquet H, Ueberheide B, Diskin R, Klein F, Oliveira TY, Pietzsch J, Fenyo D, Abadir A, Velinzon K, Hurley A, Myung S, Boulad F, Poignard P, Burton DR, Pereyra F, Ho DD, Walker BD, Seaman MS, Bjorkman PJ, Chait BT, Nussenzweig MC. Sequence and structural convergence of broad and potent HIV antibodies that mimic CD4 binding. Science. 2011; 333:1633. [PubMed: 21764753]

Schelonka RL, Ivanov II, Jung DH, Ippolito GC, Nitschke L, Zhuang Y, Gartland GL, Pelkonen J, Alt FW, Rajewsky K, Schroeder HW Jr. A single DH gene segment creates its own unique CDR-H3 repertoire and is sufficient for B cell development and immune function. J Immunol. 2005; 175:6624. [PubMed: 16272317]

Schroeder HW Jr. Similarity and divergence in the development and expression of the mouse and human antibody repertoires. Dev Comp Immunol. 2006; 30:119. [PubMed: 16083957]

Schwickert TA, Victora GD, Fooksman DR, Kamphorst AO, Mugnier MR, Gitlin AD, Dustin ML, Nussenzweig MC. A dynamic T cell-limited checkpoint regulates affinity-dependent B cell entry into the germinal center. The Journal of experimental medicine. 2011; 208:1243. [PubMed: 21576382]

Sciammas R, Shaffer AL, Schatz JH, Zhao H, Staudt LM, Singh H. Graded expression of interferon regulatory factor-4 coordinates isotype switching with plasma cell differentiation. Immunity. 2006; 25:225. [PubMed: 16919487] 
Seay K, Qi X, Zheng JH, Zhang C, Chen K, Dutta M, Deneroff K, Ochsenbauer C, Kappes JC, Littman DR, Goldstein H. Mice transgenic for CD4-specific human CD4, CCR5 and cyclin T1 expression: a new model for investigating HIV-1 transmission and treatment efficacy. PLoS One. 2013; 8:e63537. [PubMed: 23691059]

Sekiguchi DR, Jainandunsing SM, Fields ML, Maldonado MA, Madaio MP, Erikson J, Weigert M, Eisenberg RA. Chronic graft-versus-host in Ig knockin transgenic mice abrogates B cell tolerance in anti-double-stranded DNA B cells. J Immunol. 2002; 168:4142. [PubMed: 11937575]

Seo SJ, Fields ML, Buckler JL, Reed AJ, Mandik-Nayak L, Nish SA, Noelle RJ, Turka LA, Finkelman FD, Caton AJ, Erikson J. The impact of T helper and T regulatory cells on the regulation of antidouble-stranded DNA B cells. Immunity. 2002; 16:535. [PubMed: 11970877]

Shaffer JS, Moore PL, Kardar M, Chakraborty AK. Optimal immunization cocktails can promote induction of broadly neutralizing Abs against highly mutable pathogens. Proc Natl Acad Sci U S A. 2016

Sharma P, Allison JP. The future of immune checkpoint therapy. Science. 2015a; 348:56. [PubMed: 25838373]

Sharma P, Allison JP. Immune checkpoint targeting in cancer therapy: toward combination strategies with curative potential. Cell. 2015b; 161:205. [PubMed: 25860605]

Sharpe AH, Wherry EJ, Ahmed R, Freeman GJ. The function of programmed cell death 1 and its ligands in regulating autoimmunity and infection. Nat Immunol. 2007; 8:239. [PubMed: 17304234]

Shih TA, Meffre E, Roederer M, Nussenzweig MC. Role of BCR affinity in T cell dependent antibody responses in vivo. Nature immunology. 2002; 3:570. [PubMed: 12021782]

Shiokawa S, Mortari F, Lima JO, Nunez C, Bertrand FE 3rd, Kirkham PM, Zhu S, Dasanayake AP, Schroeder HW Jr. IgM heavy chain complementarity-determining region 3 diversity is constrained by genetic and somatic mechanisms until two months after birth. J Immunol. 1999; 162:6060. [PubMed: 10229847]

Shlomchik M, Mascelli M, Shan H, Radic MZ, Pisetsky D, Marshak-Rothstein A, Weigert M. AntiDNA antibodies from autoimmune mice arise by clonal expansion and somatic mutation. J Exp Med. 1990; 171:265. [PubMed: 2104919]

Shlomchik MJ. Sites and stages of autoreactive B cell activation and regulation. Immunity. 2008; 28:18. [PubMed: 18199415]

Shokat KM, Goodnow CC. Antigen-induced B-cell death and elimination during germinal-centre immune responses. Nature. 1995; 375:334. [PubMed: 7753200]

Silverman GJ, Goodyear CS. Confounding B-cell defences: lessons from a staphylococcal superantigen. Nat Rev Immunol. 2006; 6:465. [PubMed: 16724100]

Simek MD, Rida W, Priddy FH, Pung P, Carrow E, Laufer DS, Lehrman JK, Boaz M, Tarragona-Fiol T, Miiro G, Birungi J, Pozniak A, McPhee DA, Manigart O, Karita E, Inwoley A, Jaoko W, Dehovitz J, Bekker LG, Pitisuttithum P, Paris R, Walker LM, Poignard P, Wrin T, Fast PE, Burton DR, Koff WC. Human immunodeficiency virus type 1 elite neutralizers: individuals with broad and potent neutralizing activity identified by using a high-throughput neutralization assay together with an analytical selection algorithm. J Virol. 2009; 83:7337. [PubMed: 19439467]

Singh SK, Maeda K, Eid MM, Almofty SA, Ono M, Pham P, Goodman MF, Sakaguchi N. GANP regulates recruitment of AID to immunoglobulin variable regions by modulating transcription and nucleosome occupancy. Nat Commun. 2013; 4:1830. [PubMed: 23652018]

Sliepen K, Medina-Ramirez M, Yasmeen A, Moore JP, Klasse PJ, Sanders RW. Binding of inferred germline precursors of broadly neutralizing HIV-1 antibodies to native-like envelope trimers. Virology. 2015; 486:116. [PubMed: 26433050]

Sliepen K, Sanders RW. HIV-1 envelope glycoprotein immunogens to induce broadly neutralizing antibodies. Expert Rev Vaccines. 2016; 15:349. [PubMed: 26654478]

Smith KG, Light A, O'Reilly LA, Ang SM, Strasser A, Tarlinton D. bcl-2 transgene expression inhibits apoptosis in the germinal center and reveals differences in the selection of memory $\mathrm{B}$ cells and bone marrow antibody-forming cells. J Exp Med. 2000; 191:475. [PubMed: 10662793]

Sok D, Briney B, Jardine JG, Kulp DW, Menis S, Pauthner M, Wood A, Lee EC, Le KM, Jones M, Ramos A, Kalyuzhniy O, Adachi Y, Kubitz M, MacPherson S, Bradley A, Friedrich GA, Schief 
WR, Burton DR. Priming HIV-1 broadly neutralizing antibody precursors in human Ig loci transgenic mice. Science. 2016

Sok D, Laserson U, Laserson J, Liu Y, Vigneault F, Julien JP, Briney B, Ramos A, Saye KF, Le K, Mahan A, Wang S, Kardar M, Yaari G, Walker LM, Simen BB, St John EP, Chan-Hui PY, Swiderek K, Kleinstein SH, Alter G, Seaman MS, Chakraborty AK, Koller D, Wilson IA, Church GM, Burton DR, Poignard P. The effects of somatic hypermutation on neutralization and binding in the PGT121 family of broadly neutralizing HIV antibodies. PLoS Pathog. 2013; 9:e1003754. [PubMed: 24278016]

Stanfield RL, De Castro C, Marzaioli AM, Wilson IA, Pantophlet R. Crystal structure of the HIV neutralizing antibody $2 \mathrm{G} 12$ in complex with a bacterial oligosaccharide analog of mammalian oligomannose. Glycobiology. 2015; 25:412. [PubMed: 25380763]

Steichen JM, Kulp DW, Tokatlian T, Escolano A, Dosenovic P, Stanfield RL, McCoy LE, Ozorowski G, Hu X, Kalyuzhniy O, Briney B, Schiffner T, Garces F, Freund NT, Gitlin AD, Georgeson E, Kubitz M, Adachi Y, Jones M, Mutafyan A, Yun DS, Mayer C, Ward AB, Burton DR, Wilson IA, Irvine DJ, Nussenzweig MC, Schief WR. HIV vaccine design to target germline precursors of glycan-dependent broadly neutralizing antibodies. Immunity. 2106; 166:1445.

Strasser A, Whittingham S, Vaux DL, Bath ML, Adams JM, Cory S, Harris AW. Enforced BCL2 expression in B-lymphoid cells prolongs antibody responses and elicits autoimmune disease. Proc Natl Acad Sci U S A. 1991; 88:8661. [PubMed: 1924327]

Takahashi Y, Cerasoli DM, Dal Porto JM, Shimoda M, Freund R, Fang W, Telander DG, Malvey EN, Mueller DL, Behrens TW, Kelsoe G. Relaxed negative selection in germinal centers and impaired affinity maturation in bcl-xL transgenic mice. J Exp Med. 1999; 190:399. [PubMed: 10430628]

Teft WA, Kirchhof MG, Madrenas J. A molecular perspective of CTLA-4 function. Annu Rev Immunol. 2006; 24:65. [PubMed: 16551244]

Tian M, Cheng C, Chen X, Duan H, Cheng HL, Dao M, Sheng Z, Kimble M, Wang L, Lin S, Schmidt SD, Du Z, Joyce MG, Chen Y, DeKosky BJ, Chen Y, Normandin E, Cantor E, Chen RE, DoriaRose N, Zhang Y, Shi W, Kong WP, Choe M, Henry AR, Laboune F, Georgiev IS, Huang PY, Jain S, McGuire A, Georgeson E, Menis S, Douek DC, Schief WR, Stamatatos L, Kwong PD, Shapiro L, Haynes BF, Mascola JR, Alt FW. Induction of HIV neutralizing antibody lineages in mice with diverse precursor repertoires. Cell. 2016; 166:1471. [PubMed: 27610571]

Tiegs SL, Russell DM, Nemazee D. Receptor editing in self-reactive bone marrow B cells. J Exp Med. 1993; 177:1009. [PubMed: 8459201]

Tiller T, Tsuiji M, Yurasov S, Velinzon K, Nussenzweig MC, Wardemann H. Autoreactivity in human IgG+ memory B cells. Immunity. 2007; 26:205. [PubMed: 17306569]

Tomaras GD, Binley JM, Gray ES, Crooks ET, Osawa K, Moore PL, Tumba N, Tong T, Shen X, Yates NL, Decker J, Wibmer CK, Gao F, Alam SM, Easterbrook P, Abdool Karim S, Kamanga G, Crump JA, Cohen M, Shaw GM, Mascola JR, Haynes BF, Montefiori DC, Morris L. Polyclonal B cell responses to conserved neutralization epitopes in a subset of HIV-1-infected individuals. Journal of virology. 2011; 85:11502. [PubMed: 21849452]

Tomaras GD, Ferrari G, Shen X, Alam SM, Liao HX, Pollara J, Bonsignori M, Moody MA, Fong Y, Chen X, Poling B, Nicholson CO, Zhang R, Lu X, Parks R, Kaewkungwal J, Nitayaphan S, Pitisuttithum P, Rerks-Ngarm S, Gilbert PB, Kim JH, Michael NL, Montefiori DC, Haynes BF. Vaccine-induced plasma IgA specific for the $\mathrm{C} 1$ region of the HIV-1 envelope blocks binding and effector function of IgG. Proc Natl Acad Sci U S A. 2013; 110:9019. [PubMed: 23661056]

Tong T, Crooks ET, Osawa K, Binley JM. HIV-1 virus-like particles bearing pure env trimers expose neutralizing epitopes but occlude nonneutralizing epitopes. J Virol. 2012; 86:3574. [PubMed: 22301141]

Turan S, Zehe C, Kuehle J, Qiao J, Bode J. Recombinase-mediated cassette exchange (RMCE) - a rapidly-expanding toolbox for targeted genomic modifications. Gene. 2013; 515:1. [PubMed: 23201421]

Vcelar B, Stiegler G, Wolf HM, Muntean W, Leschnik B, Mehandru S, Markowitz M, Armbruster C, Kunert R, Eibl MM, Katinger H. Reassessment of autoreactivity of the broadly neutralizing HIV antibodies 4E10 and 2F5 and retrospective analysis of clinical safety data. Aids. 2007; 21:2161. [PubMed: 18090042] 
Verkoczy L, Chen Y, Bouton-Verville H, Zhang J, Diaz M, Hutchinson J, Ouyang YB, Alam SM, Holl TM, Hwang KK, Kelsoe G, Haynes BF. Rescue of HIV-1 broad neutralizing antibody-expressing B cells in 2F5 VH x VL knockin mice reveals multiple tolerance controls. J Immunol. 2011a; 187:3785. [PubMed: 21908739]

Verkoczy L, Chen Y, Zhang J, Bouton-Verville H, Newman A, Lockwood B, Scearce RM, Montefiori DC, Dennison SM, Xia SM, Hwang KK, Liao HX, Alam SM, Haynes BF. Induction of HIV-1 broad neutralizing antibodies in 2F5 knock-in mice: selection against membrane proximal external region-associated autoreactivity limits T-dependent responses. J Immunol. 2013; 191:2538. [PubMed: 23918977]

Verkoczy L, Diaz M. Autoreactivity in HIV-1 broadly neutralizing antibodies: implications for their function and induction by vaccination. Curr Opin HIV AIDS. 2014; 9:224. [PubMed: 24714565]

Verkoczy L, Diaz M, Holl TM, Ouyang YB, Bouton-Verville H, Alam SM, Liao HX, Kelsoe G, Haynes BF. Autoreactivity in an HIV-1 broadly reactive neutralizing antibody variable region heavy chain induces immunologic tolerance. Proc Natl Acad Sci U S A. 2010; 107:181. [PubMed: 20018688]

Verkoczy L, Duong B, Skog P, Ait-Azzouzene D, Puri K, Vela JL, Nemazee D. Basal B cell receptordirected phosphatidylinositol 3-kinase signaling turns off RAGs and promotes B cell-positive selection. J Immunol. 2007; 178:6332. [PubMed: 17475862]

Verkoczy L, Kelsoe G, Haynes BF. HIV-1 envelope gp41 broadly neutralizing antibodies: hurdles for vaccine development. PLoS Pathog. 2014; 10:e1004073. [PubMed: 24853821]

Verkoczy L, Kelsoe G, Moody MA, Haynes BF. Role of immune mechanisms in induction of HIV-1 broadly neutralizing antibodies. Curr Opin Immunol. 2011b; 23:383. [PubMed: 21524897]

Vettermann C, Jack HM. The pre-B cell receptor: turning autoreactivity into self-defense. Trends Immunol. 2010; 31:176. [PubMed: 20356792]

Victor Garcia J. Humanized mice for HIV and AIDS research. Curr Opin Virol. 2016; 19:56. [PubMed: 27447446]

von Boehmer H, Melchers F. Checkpoints in lymphocyte development and autoimmune disease. Nat Immunol. 2010; 11:14. [PubMed: 20016505]

Walker LM, Huber M, Doores KJ, Falkowska E, Pejchal R, Julien JP, Wang SK, Ramos A, Chan-Hui PY, Moyle M, Mitcham JL, Hammond PW, Olsen OA, Phung P, Fling S, Wong CH, Phogat S, Wrin T, Simek MD, Koff WC, Wilson IA, Burton DR, Poignard P. Broad neutralization coverage of HIV by multiple highly potent antibodies. Nature. 2011; 477:466. [PubMed: 21849977]

Wang H, Shlomchik MJ. High affinity rheumatoid factor transgenic B cells are eliminated in normal mice. J Immunol. 1997; 159:1125. [PubMed: 9233605]

Wang S, Mata-Fink J, Kriegsman B, Hanson M, Irvine DJ, Eisen HN, Burton DR, Wittrup KD, Kardar M, Chakraborty AK. Manipulating the selection forces during affinity maturation to generate cross-reactive HIV antibodies. Cell. 2015; 160:785. [PubMed: 25662010]

Wardemann H, Yurasov S, Schaefer A, Young JW, Meffre E, Nussenzweig MC. Predominant autoantibody production by early human B cell precursors. Science. 2003; 301:1374. [PubMed: 12920303]

Wei X, Decker JM, Wang S, Hui H, Kappes JC, Wu X, Salazar-Gonzalez JF, Salazar MG, Kilby JM, Saag MS, Komarova NL, Nowak MA, Hahn BH, Kwong PD, Shaw GM. Antibody neutralization and escape by HIV-1. Nature. 2003; 422:307. [PubMed: 12646921]

West AP Jr, Scharf L, Scheid JF, Klein F, Bjorkman PJ, Nussenzweig MC. Structural insights on the role of antibodies in HIV-1 vaccine and therapy. Cell. 2014; 156:633. [PubMed: 24529371]

Willis JR, Finn JA, Briney B, Sapparapu G, Singh V, King H, LaBranche CC, Montefiori DC, Meiler J, Crowe JE Jr. Long antibody HCDR3s from HIV-naive donors presented on a PG9 neutralizing antibody background mediate HIV neutralization. Proc Natl Acad Sci U S A. 2016; 113:4446. [PubMed: 27044078]

Wu X, Yang ZY, Li Y, Hogerkorp CM, Schief WR, Seaman MS, Zhou T, Schmidt SD, Wu L, Xu L, Longo NS, McKee K, O’Dell S, Louder MK, Wycuff DL, Feng Y, Nason M, Doria-Rose N, Connors M, Kwong PD, Roederer M, Wyatt RT, Nabel GJ, Mascola JR. Rational design of envelope identifies broadly neutralizing human monoclonal antibodies to HIV-1. Science. 2010; 329:856. [PubMed: 20616233] 
Wu X, Zhang Z, Schramm CA, Joyce MG, Kwon YD, Zhou T, Sheng Z, Zhang B, O’Dell S, McKee K, Georgiev IS, Chuang GY, Longo NS, Lynch RM, Saunders KO, Soto C, Srivatsan S, Yang Y, Bailer RT, Louder MK, Mullikin JC, Connors M, Kwong PD, Mascola JR, Shapiro L. Maturation and Diversity of the VRC01-Antibody Lineage over 15 Years of Chronic HIV-1 Infection. Cell. 2015; 161:470. [PubMed: 25865483]

Wu X, Zhou T, Zhu J, Zhang B, Georgiev I, Wang C, Chen X, Longo NS, Louder M, McKee K, O'Dell S, Perfetto S, Schmidt SD, Shi W, Wu L, Yang Y, Yang ZY, Yang Z, Zhang Z, Bonsignori M, Crump JA, Kapiga SH, Sam NE, Haynes BF, Simek M, Burton DR, Koff WC, Doria-Rose NA, Connors M, Mullikin JC, Nabel GJ, Roederer M, Shapiro L, Kwong PD, Mascola JR. Focused evolution of HIV-1 neutralizing antibodies revealed by structures and deep sequencing. Science. 2011; 333:1593. [PubMed: 21835983]

Xiao X, Chen W, Feng Y, Zhu Z, Prabakaran P, Wang Y, Zhang MY, Longo NS, Dimitrov DS. Germline-like predecessors of broadly neutralizing antibodies lack measurable binding to HIV-1 envelope glycoproteins: implications for evasion of immune responses and design of vaccine immunogens. Biochem Biophys Res Commun. 2009; 390:404. [PubMed: 19748484]

Yang G, Holl TM, Liu Y, Li Y, Lu X, Nicely NI, Kepler TB, Alam SM, Liao HX, Cain DW, Spicer L, VandeBerg JL, Haynes BF, Kelsoe G. Identification of autoantigens recognized by the 2F5 and 4E10 broadly neutralizing HIV-1 antibodies. J Exp Med. 2013; 210:241. [PubMed: 23359068]

Yeap LS, Hwang JK, Du Z, Meyers RM, Meng FL, Jakubauskaite A, Liu M, Mani V, Neuberg D, Kepler TB, Wang JH, Alt FW. Sequence-Intrinsic Mechanisms that Target AID Mutational Outcomes on Antibody Genes. Cell. 2015; 163:1124. [PubMed: 26582132]

Yu RK, Ariga T, Usuki S, Kaida K. Pathological roles of ganglioside mimicry in Guillain-Barre syndrome and related neuropathies. Adv Exp Med Biol. 2011; 705:349. [PubMed: 21618117]

Zan H, Komori A, Li Z, Cerutti A, Schaffer A, Flajnik MF, Diaz M, Casali P. The translesion DNA polymerase zeta plays a major role in Ig and bcl-6 somatic hypermutation. Immunity. 2001; 14:643. [PubMed: 11371365]

Zemlin M, Klinger M, Link J, Zemlin C, Bauer K, Engler JA, Schroeder HW Jr, Kirkham PM. Expressed murine and human CDR-H3 intervals of equal length exhibit distinct repertoires that differ in their amino acid composition and predicted range of structures. J Mol Biol. 2003; 334:733. [PubMed: 14636599]

Zhang J, Alam SM, Bouton-Verville H, Chen Y, Newman A, Stewart S, Jaeger FH, Montefiori DC, Dennison SM, Haynes BF, Verkoczy L. Modulation of nonneutralizing HIV-1 gp41 responses by an MHC-restricted TH epitope overlapping those of membrane proximal external region broadly neutralizing antibodies. J Immunol. 2014; 192:1693. [PubMed: 24465011]

Zhang J, Shakhnovich EI. Optimality of mutation and selection in germinal centers. PLoS Comput Biol. 2010; 6:e1000800. [PubMed: 20532164]

Zhang R, Verkoczy L, Wiehe K, Munir Alam S, Nicely NI, Santra S, Bradley T, Pemble CWt, Zhang J, Gao F, Montefiori DC, Bouton-Verville H, Kelsoe G, Larimore K, Greenberg PD, Parks R, Foulger A, Peel JN, Luo K, Lu X, Trama AM, Vandergrift N, Tomaras GD, Kepler TB, Moody MA, Liao HX, Haynes BF. Initiation of immune tolerance-controlled HIV gp41 neutralizing B cell lineages. Sci Transl Med. 2016; 8:336ra62.

Zhang Y, Su SC, Hecox DB, Brady GF, Mackin KM, Clark AG, Foster MH. Central tolerance regulates B cells reactive with Goodpasture antigen alpha3(IV)NC1 collagen. J Immunol. 2008; 181:6092. [PubMed: 18941198]

Zhou T, Georgiev I, Wu X, Yang ZY, Dai K, Finzi A, Do Kwon Y, Scheid JF, Shi W, Xu L, Yang Y, Zhu J, Nussenzweig MC, Sodroski J, Shapiro L, Nabel GJ, Mascola JR, Kwong PD. Structural basis for broad and potent neutralization of HIV-1 by antibody VRC01. Science. 2010; 329:811. [PubMed: 20616231]

Zhou T, Lynch RM, Chen L, Acharya P, Wu X, Doria-Rose NA, Joyce MG, Lingwood D, Soto C, Bailer RT, Ernandes MJ, Kong R, Longo NS, Louder MK, McKee K, O’Dell S, Schmidt SD, Tran L, Yang Z, Druz A, Luongo TS, Moquin S, Srivatsan S, Yang Y, Zhang B, Zheng A, Pancera M, Kirys T, Georgiev IS, Gindin T, Peng HP, Yang AS, Mullikin JC, Gray MD, Stamatatos L, Burton DR, Koff WC, Cohen MS, Haynes BF, Casazza JP, Connors M, Corti D, Lanzavecchia A, Sattentau QJ, Weiss RA, West AP Jr, Bjorkman PJ, Scheid JF, Nussenzweig 
MC, Shapiro L, Mascola JR, Kwong PD. Structural Repertoire of HIV-1-Neutralizing Antibodies Targeting the CD4 Supersite in 14 Donors. Cell. 2015; 161:1280. [PubMed: 26004070]

Zhou T, Zhu J, Wu X, Moquin S, Zhang B, Acharya P, Georgiev IS, Altae-Tran HR, Chuang GY, Joyce MG, Do Kwon Y, Longo NS, Louder MK, Luongo T, McKee K, Schramm CA, Skinner J, Yang Y, Yang Z, Zhang Z, Zheng A, Bonsignori M, Haynes BF, Scheid JF, Nussenzweig MC, Simek M, Burton DR, Koff WC, Mullikin JC, Connors M, Shapiro L, Nabel GJ, Mascola JR, Kwong PD. Multidonor analysis reveals structural elements, genetic determinants, and maturation pathway for HIV-1 neutralization by VRC01-class antibodies. Immunity. 2013; 39:245.

[PubMed: 23911655] 


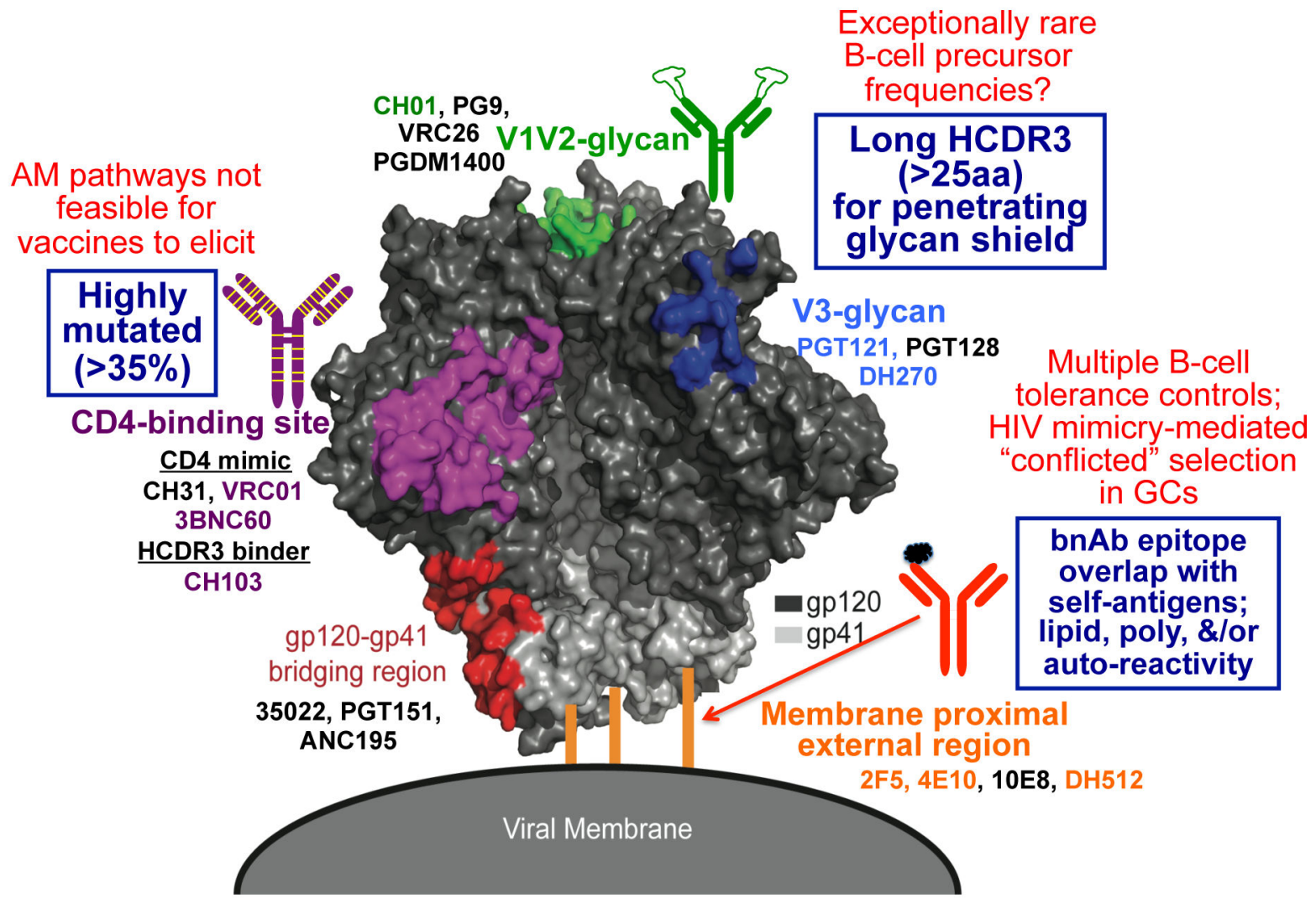

Fig. 1. Varied penetrance of rare (but desirable) traits in bnAbs to distinct Env regions Shown is a schematic representation of the HIV pre-fusion trimer structure (Pancera et al., 2014, Nature 514:455), showing its gp120 and gp41 protomers. Below each of the major common regions targeted by bnAbs, the V2-glycan apex (green), V3 glycan high mannose patch (blue), the CD4bs (magenta), the gp41 MPER (orange), and the gp120-gp41 bridging region (red), are listed prototype $\mathrm{mAb}$ bnAbs for that site, including ones (highlighted in color) for which bnAb KI models have been made (or are under construction). Next to each of the four better-characterized Env sites are the most penetrant functional trait in the corresponding bnAb class (in blue boxes), and above it (in red letters) is listed the major anticipated immunologic hurdle for vaccination to overcome. 

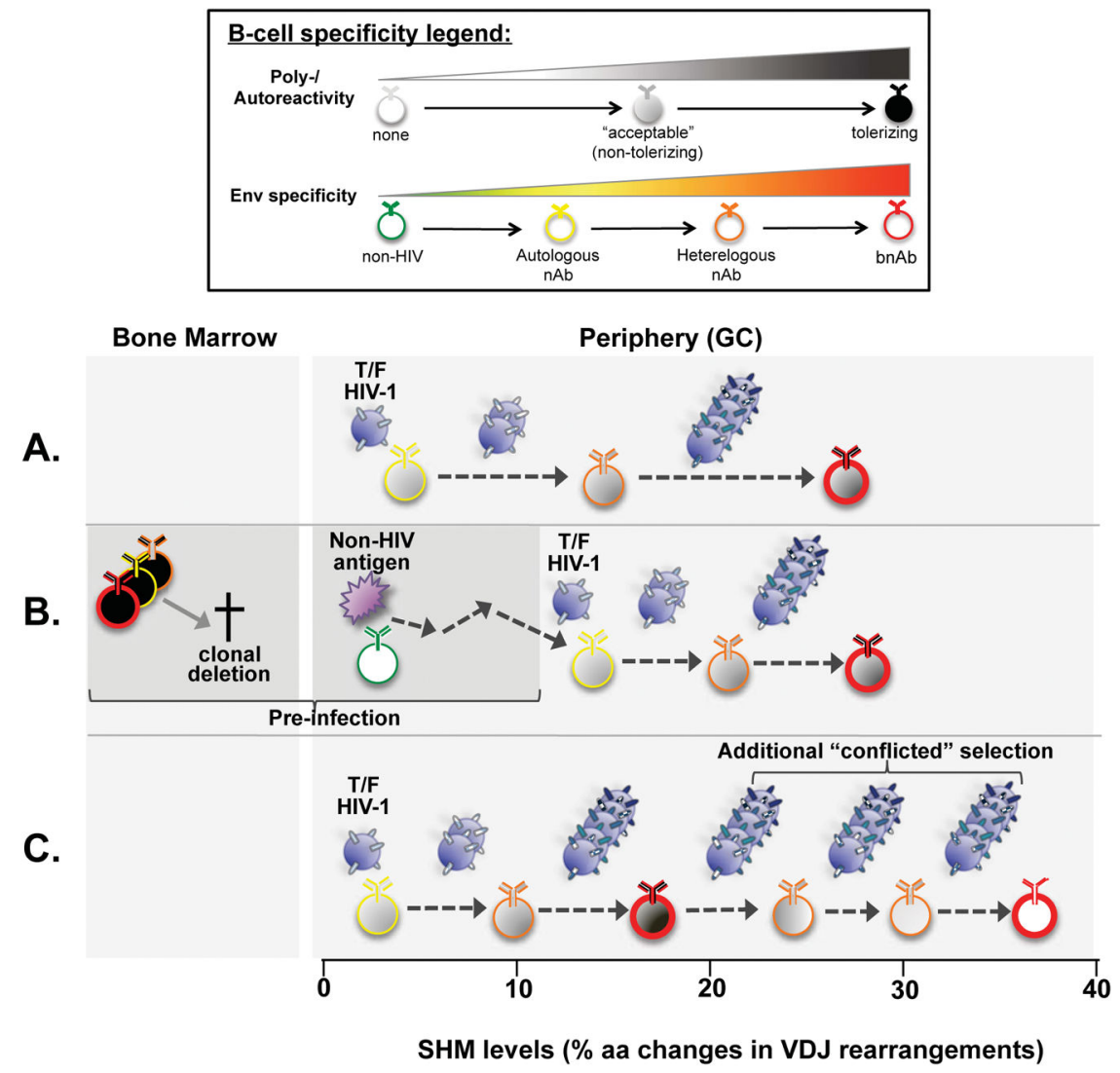

Fig. 2. Potential "vaccine-relevant" mechanisms driving high SHM levels in bnAbs that develop during prolonged HIV-1 infection

In a "non-tolerance" model (A), GC B cells with no/minimal reactivity to mature Envs interact with founder/transmitted Envs and undergo extensive AM, driven by viral escape/Env diversification pressure, which demands difficult structural modifications to achieve both FRW flexibility and to accommodate finer variation in otherwise conserved Env epitopes. In models where HIV self-mimicry impacts SHM, i.e. (B) and (C), this could occur either as an indirect or direct result of tolerization. In a model where tolerizing selfreactivity indirectly impacts excess SHM (B), B-cells with bnAb epitopes that mimic host antigens (e.g. 2F5 ELDKWA minimal epitope) are clonally deleted in bone marrow development, creating "holes in the repertoire". In the absence of competition, weakly crossreactive clones that already underwent non-Env driven AM can then be recruited into additional, Env-driven AM pathways. Alternatively, in a model where self-tolerance directly drives excess SHM (C), B-cells that acquire both self and bnAb specificity via AM use additional rounds of $\mathrm{SHM} /$ selection to retain bnAb specificity, while removing tolerizing self-reactivity. In this model, the degree of additional selection/SHM would be proportional to how closely a particular BnAb epitope mimics self-antigen(s). Note that all three hypotheses are not mutually exclusive, and the combined mechanisms illustrated, could account for the especially tortuous pathways of certain bnAb lineages. Figure reproduced with permission, from Verkoczy and Diaz, Curr Opin HIV AIDS, 2014, 9:224. 


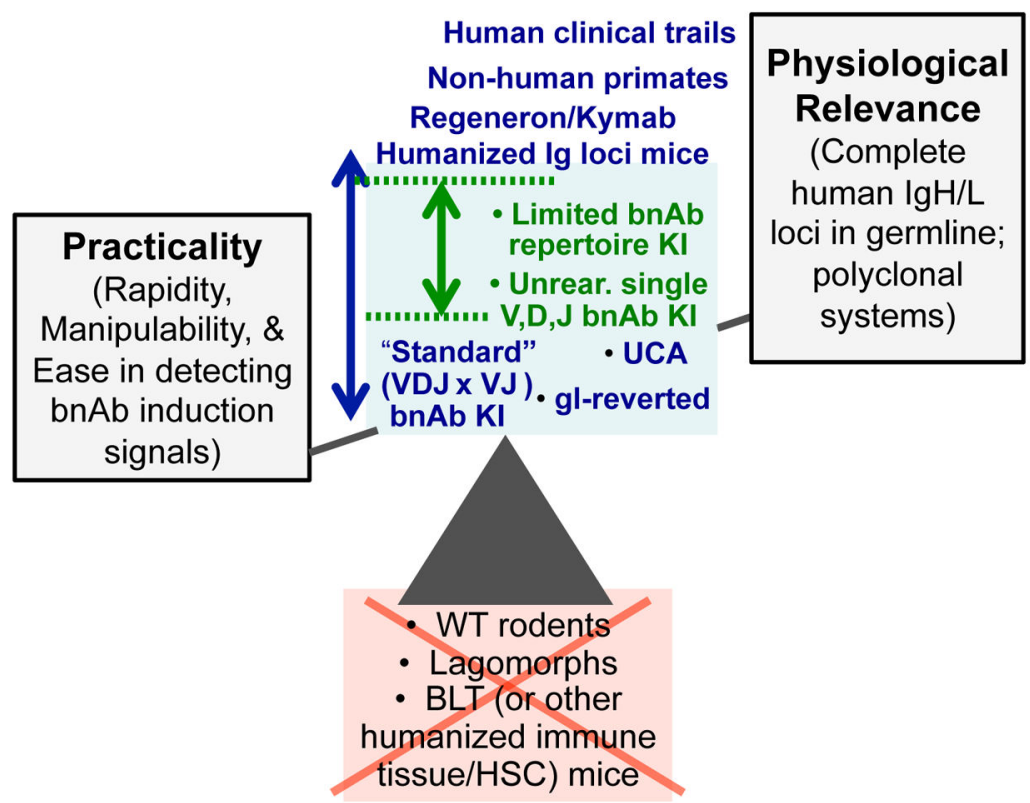

Fig. 3. Comparison of animal models for evaluating bnAb-targeting vaccine regimens Schematic representation of relative practicality and physiological relevance of various current and planned animal models for testing vaccines aimed at eliciting bnAb responses. Optimal model seek to find the can best bridge gap between practicality of detection afforded by standard UCA KI frequencies, with the physiological relevance of human unrearranged immune loci. Acceptable current versions with "sufficient" balance of both sets of required features are shown in blue, and include existing $1^{\text {st }}$ and early $2^{\text {nd }}$ generation KI models; "Optimal" platforms for the best balance of iterative, yet physiologically relevant testing (" 2 nd and $3^{\text {rd }}$ generation bnAb KI platforms; see Section 6 and Figure 10 for more detail) are shown in green. Shown in red are models not thought to be practical for these types of studies. 


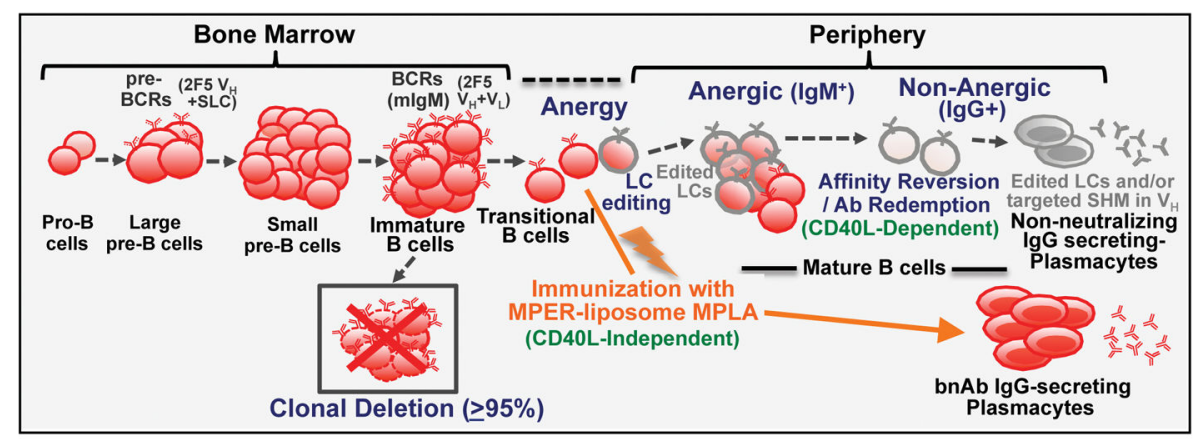

Fig. 4. B-cell tolerance controls in the 2F5 bnAb KI mouse model

Pictorial summary of bnAb regulation and induction studies done in the setting of $2 \mathrm{~F} 5$ $\mathrm{V}_{\mathrm{H}} \mathrm{DJ}_{\mathrm{H}}^{+/+}+\mathrm{V} \kappa \mathrm{J}^{+/+} \mathrm{KI}$ mice. The original (affinity matured) version of $2 \mathrm{~F} 5$, upon knocking its VDJ/VJ rearrangements into B-cells, fails to pass multiple tolerance checkpoints (Chen et al., 2013b; Verkoczy et al., 2011a; Verkoczy et al., 2010). However, while nearly all 2F5 ${ }^{+}$Bcells are clonally deleted at the 1 st checkpoint (the pre->imm. B transition), 5\% populate the periphery, but most have been functionally silenced (rendered "anergic"). Amongst these residual anergic B-cells, a small subset can remove all self-reactivity \& escape tolerance altogether, but due to the inability of 2F5 KI LC-targeting receptor editing to remove dominant 2F5 HC self-reactivity, purifying selection occurs later in development, by a Tdependent, SHM-driven process, “Affinity Reversion/Ab Redemption” (Haynes, Verkoczy and Kelsoe, 2014; Verkoczy et al., 2013; Verkoczy and Diaz, 2014). Finally, serum bnAb IgG responses can be elicited in 2F5 KI mice immunized with an MPER peptide-lipid-TLR agonist vaccine regime through re-activation of the unedited $\left(\mathrm{MPER}^{+}\right)$anergic KI B cells not yet subjected to purifying selection (Chen et al., 2013b; Verkoczy et al., 2011a). Adapted with permission, from Verkoczy, Alt, and Tian, Immunol Rev, 2017, In Press. 

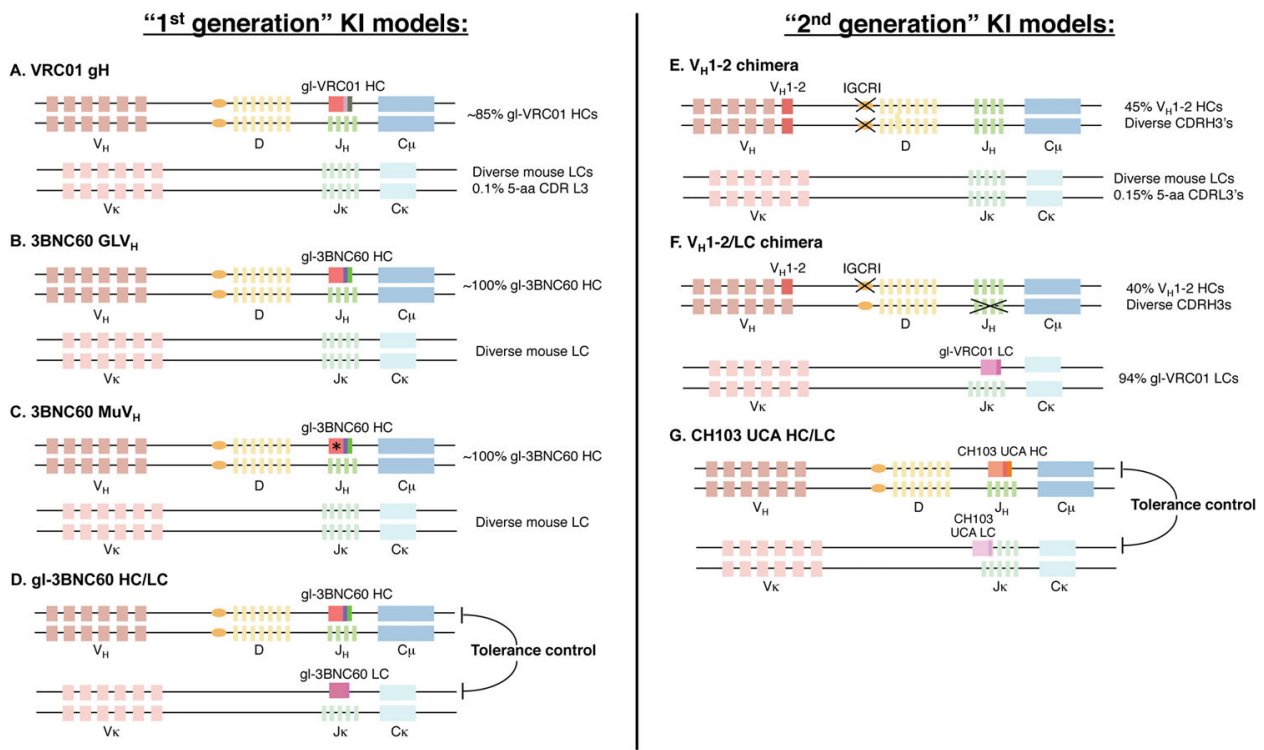

Fig. 5. Comparison of various current mouse bnAb KI/chimeric models for testing CD4bs bnAbtargeting vaccine strategies

Schematic illustration of " 1 st generation" VRC01gH, 3BNC60 GLV ${ }_{\mathrm{H}}$, 3BNC60 MuV $\mathrm{H}$, or gl-3BNC60 HC/LC mouse KI, or " $2^{\text {nd }}$ generation" CH103 UCA heterozygous HC/LC and $\mathrm{V}_{\mathrm{H}} 1-2$ (or $\mathrm{V}_{\mathrm{H}} 1-2 / \mathrm{LC}$ ) chimeric model alleles generated to evaluate the ability of CD4bstargeting sequential immunization strategies to induce VRC01-like Abs. Also shown are estimated precursor frequencies in the periphery and the main tolerance mechanisms involved (if any). Note the subtle, yet immunologically key, differences between the prererranged models, most notably, the UCA vs. reverted rearrangements used, and the partial removal of the Jx LC cluster in the CH103 UCA dKI model, versus its complete removal in the other KI models. Adapted with permission, from Verkoczy, Alt, and Tian, Immunol Rev, 2017, In Press. 


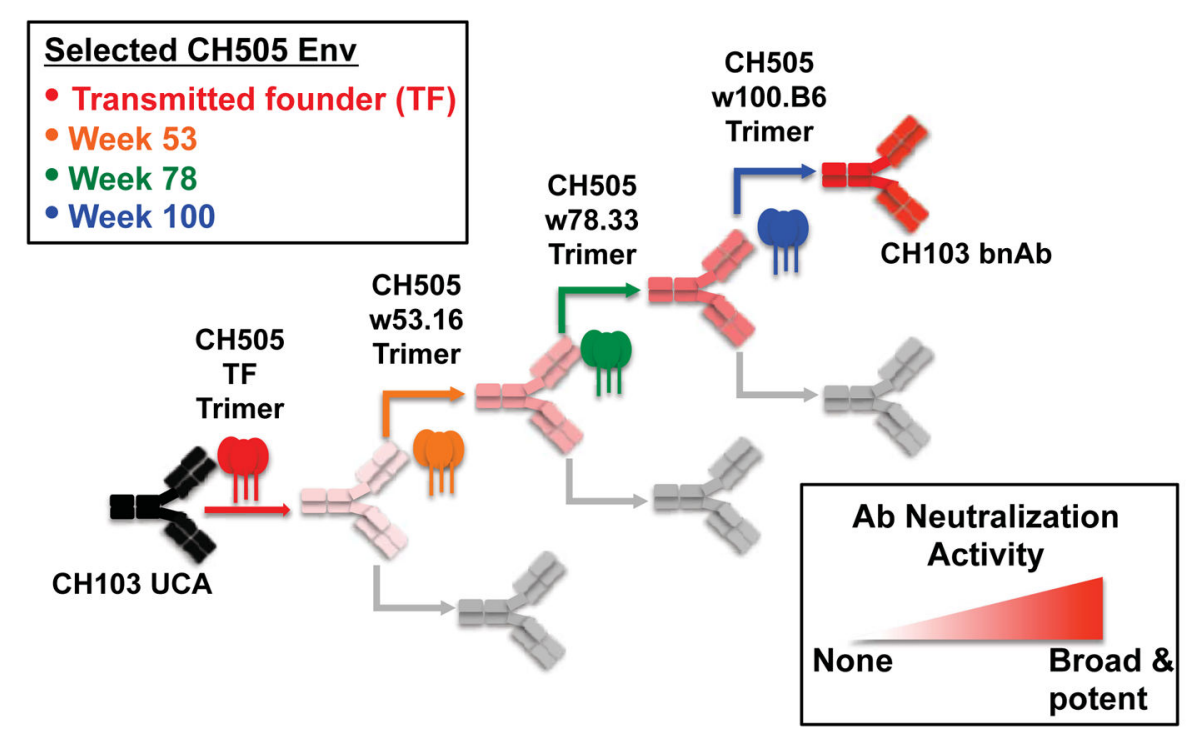

Fig. 6. Patient-based sequential vaccination for targeting bnAb B cell lineages

The CH505 "4-valent" vaccine regimen shown here as an example, is based on the B-cell Lineage design-based strategy first proposed in Haynes et al. 2012 Nat Biotech, and is comprised of the HIV-1 Envs from the transmitted founder (TF), week 53, week 78, and week 100 time-of-infection viruses in the $\mathrm{CH} 505$ patient from which the $\mathrm{CH} 103 \mathrm{bnAb}$ lineage was elucidated (Liao et al. 2013). These four viruses co-evolved with Abs that eventually developed broad neutralization and thus the basic concept is to administer these Envs in the same sequentially, in order to recreate by vaccination, the Ab maturation that occurred during natural infection. Figure is kindly provided by Kevin O. Saunders, and is adapted with permission, from Mascola and Haynes, Immunol. Rev., 2013, 254:225. 


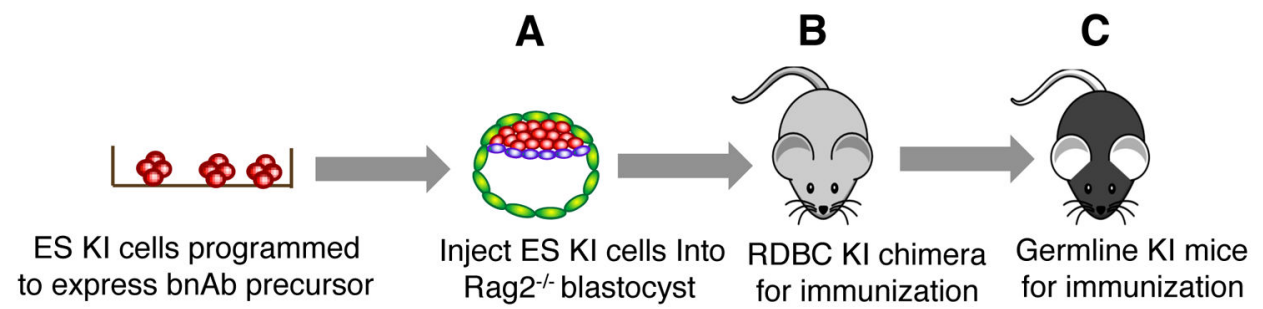

Fig. 7. High-throughput in vivo expression of bnAbs in chimeric mice using RDBC

Shown are the two major steps in the RAG-dependent blastocyst complementation (RDBC) approach (Chen et al., 1993; Chen et al., 1994; Tian et al., 2016) for generating bnAb KI chimeric mice. In the first step (A) blastocyst chimeras are generated by microinjection of bnAb KI ES cells. Then, in the second step (B), blastocyst are implanted into pseudopregnant $\mathrm{RAG}^{-/-}$foster mothers, resulting in chimeric offspring, whose lymphocytes all express the knocked-in bnAb specificity and can be directly utilized for immunization. Shown as an optional step $(\mathbf{C})$ is germline transmission of bnAb KI allele to generate a standard KI model, which can be pursued if more in-depth studies with a particular model are desired. Reproduced with permission, from Verkoczy, Alt, and Tian, Immunol Rev, 2017, In Press. 


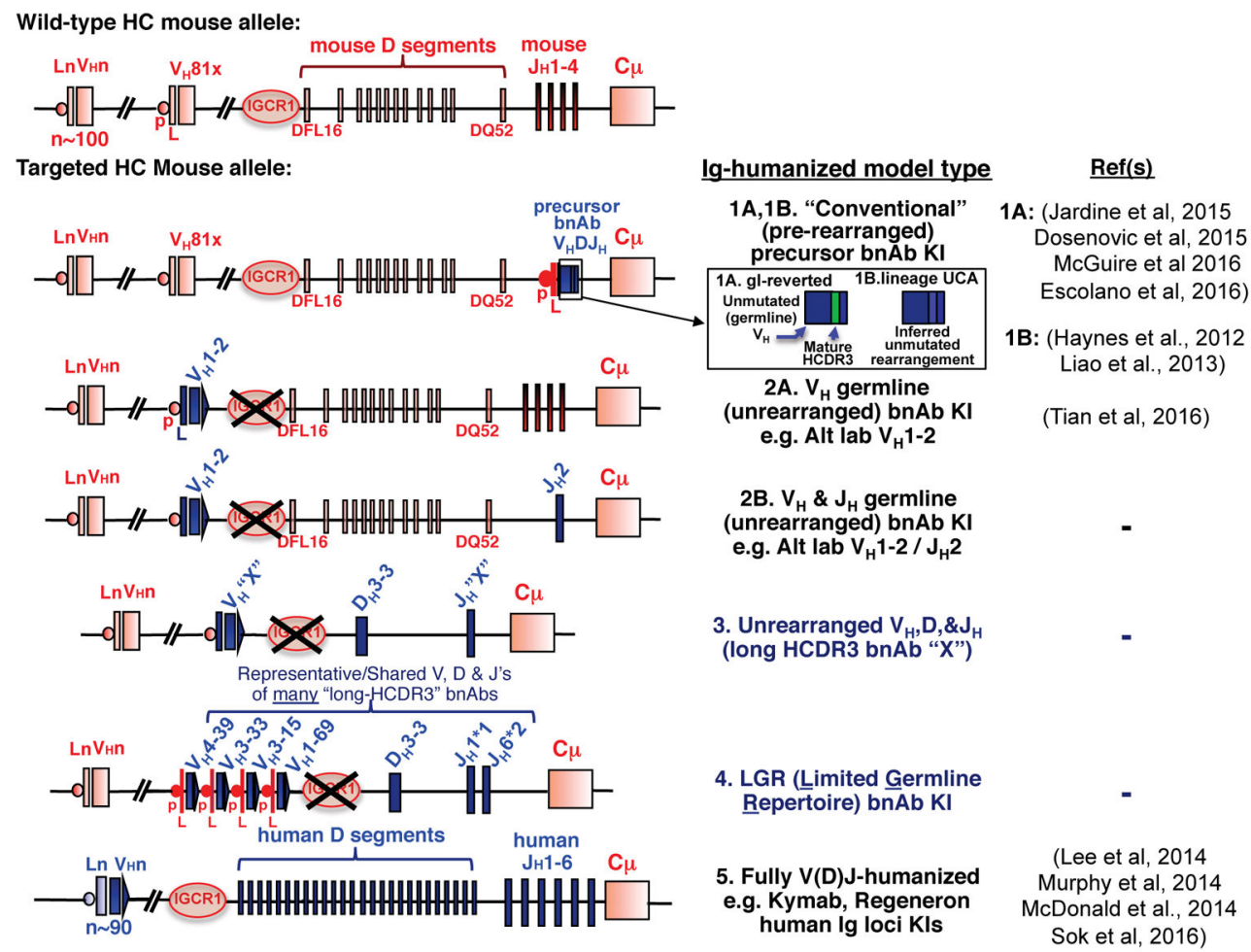

Fig. 8. Types of Ig-humanized mouse models to study vaccine-guided bnAb development Pictorial representation of the structural organization of targeted alleles from various kinds of current or planned Ig-humanized models are shown, relative to the WT murine allele. For simplicity, only HC loci are depicted, at only at one allele, and the mouse IgH locus, which spans $3 \mathrm{mb}$, is not drawn to scale in order to emphasize the key genetic elements. Models that have been published (with corresponding references to the right), or models which are being generated, are denoted in black text (Models 1, 2, and 5), while theoretical germline/ unrearranged models (that could be made or are being planned, to further bridge the current gap between physiological relevance and practicality to study bnAb specificities with long HCDR3s, thus arte difficult to infer; Models 4 and 5), are annotated in blue text. Mouse and human germline-derived sequences/segments are depicted in red and blue, respectively. Denoted as a green segment in the 1A type of models are "mutated" HCDR3s from the human bnAbs VRC01 and PGT121, i.e. from either the original/mature VRC01 bnAb, but with a single mutation to remove an unpaired cysteine (Jardine et al., 2015), or for PGT121, from the least mutated lineage member (Escolano et al., 2016). The intergenic control region 1 (IGCR1) normally found in WT HC loci is denoted by a large circle, which has been removed in models 2-4, to further facilitate rearrangement of D-proximal $\left(\mathrm{V}_{\mathrm{H}} 81 \mathrm{X}\right.$ positioned) $\mathrm{V}_{\mathrm{H}}$ segment(s) of interest. $\mathrm{p}=$ =promoter region; $\mathrm{L}=$ leader sequence. Adapted with permission, from Verkoczy, Alt, and Tian, Immunol Rev, 2017, In Press. 


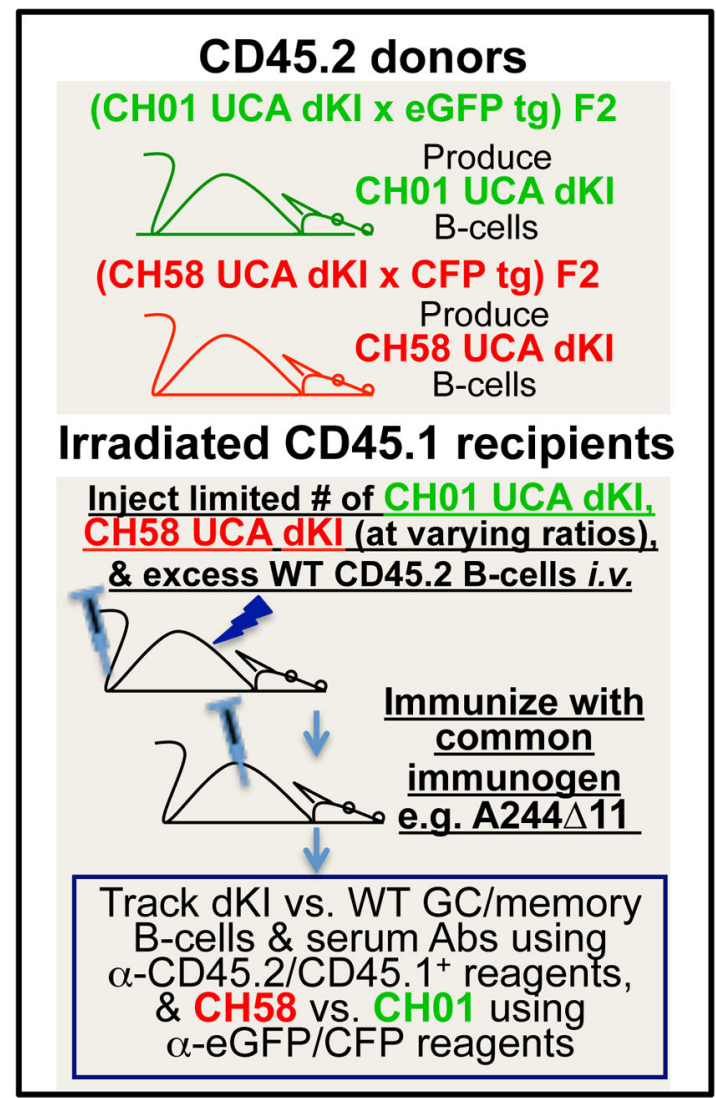

Fig. 9. Dual adoptive transfer strategy to study simultaneous in vivo priming and/or maturation of dKI B-cells expressing precursors of $\mathrm{V2}^{+} \mathrm{CH58}$ and $\mathrm{CH01}$ lineages

Given the possibility of clonal competition impacting vaccination outcomes in dKI models, the degree of competition can be manipulated via co-adoptive transfers strategies in which distinct bnAb UCA+ B-cells and WT donor B-cells can be used to reconstitute recipients. Thus, the immunization outcomes of bnAb lineage precursors with overlapping Env epitopes (in this case $\mathrm{CH} 58$ and $\mathrm{CH} 01$ ), can be compared in a polyclonal in vivo setting, in response

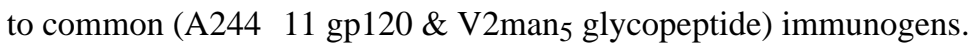


로을

\begin{tabular}{|c|c|c|c|c|c|c|c|c|c|c|c|c|c|}
\hline & 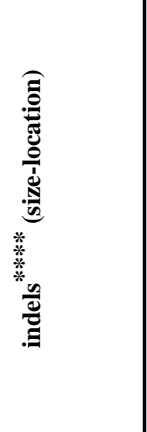 & 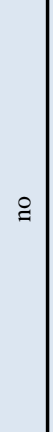 & 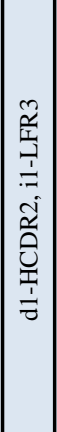 & $\frac{\mathfrak{z}}{\mathrm{z}}$ & $\frac{n}{z}$ & 芫 & 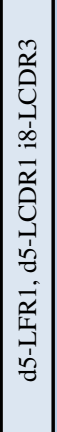 & 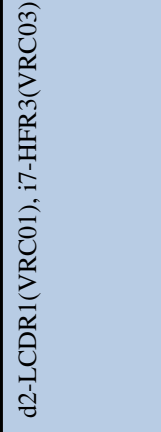 & 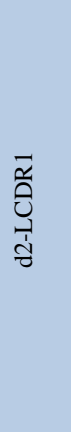 & 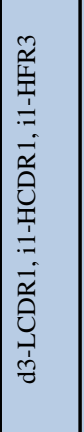 & 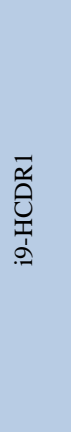 & 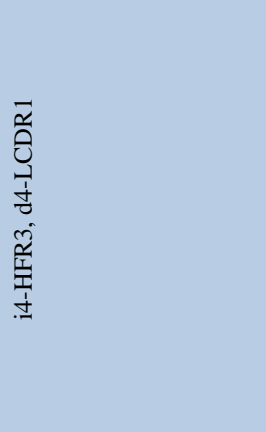 & 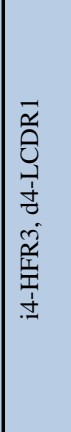 \\
\hline & 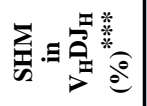 & ¿ิ & $\hat{m}$ & 产 & $\begin{array}{c}\bar{\Xi} \\
\infty \\
-\end{array}$ & ते & $\begin{array}{l}\widehat{\Xi} \\
\text { ปñ }\end{array}$ & \begin{tabular}{l}
$\infty$ \\
\multirow{f}{f}{} \\
+
\end{tabular} & $\bar{F}$ & $\mid \begin{array}{c}\hat{\rho} \\
\infty \\
\infty\end{array}$ & के & $\underset{m}{\frac{q}{m}}$ & $\underset{m}{\frac{q}{m}}$ \\
\hline 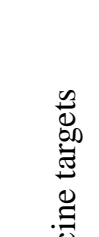 & 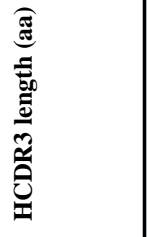 & $\stackrel{\infty}{\sim}$ & $\cong$ & $\bar{\sim}$ & 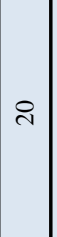 & 9 & $\bar{N}$ & $\underset{I}{ \pm}$ & $\varrho$ & \pm & $\cong$ & 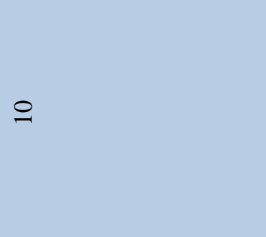 & $\cong$ \\
\hline 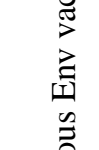 & 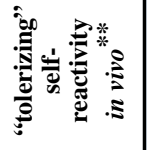 & 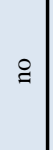 & 之 & $\mathrm{z}$ & $\mathrm{z}$ & 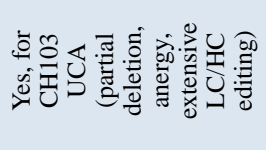 & 立 & 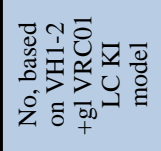 & 之 & $\hat{z}$ & そ & 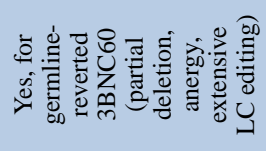 & 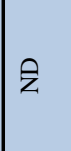 \\
\hline 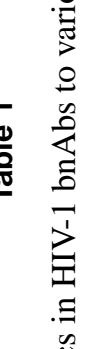 & 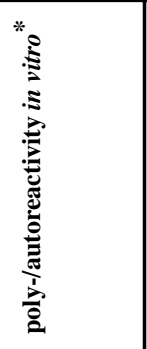 & 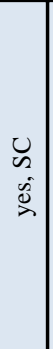 & ž & 号 & 竧 & 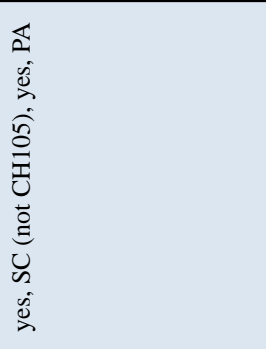 & 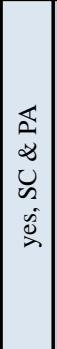 & 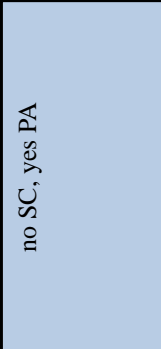 & $\begin{array}{l}\overleftarrow{1} \\
\infty \\
0 \\
0 \\
\infty \\
\infty \\
\infty\end{array}$ & $\begin{array}{l}\text { D } \\
\text { D. } \\
\text { S. }\end{array}$ & 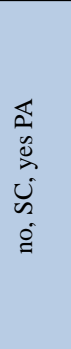 & 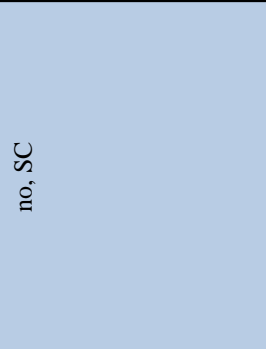 & $\begin{array}{l}\text { U } \\
\stackrel{0}{\infty}\end{array}$ \\
\hline 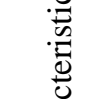 & 5 & $\begin{array}{l}\stackrel{\hat{\Upsilon}}{ } \\
\tilde{\varkappa}\end{array}$ & $\vec{f}$ & $\begin{array}{l} \pm \\
\dot{J} \\
\vec{J}\end{array}$ & $\frac{a}{\frac{i}{x}}$ & $\vec{m}$ & 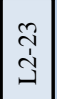 & \begin{tabular}{|l}
$\stackrel{\Upsilon}{1}$ \\
$\check{2}$
\end{tabular} & $\begin{array}{l}\text { ते } \\
\check{\check{\beth}}\end{array}$ & \begin{tabular}{|l}
$\stackrel{\hat{1}}{1}$ \\
$\tilde{2}$
\end{tabular} & $\frac{\hat{r}}{\underline{x}}$ & $\frac{\tilde{r}}{\underline{x}}$ & $\frac{\tilde{r}}{\bar{q}}$ \\
\hline$\frac{\tilde{J}}{0}$ & \pm & $I$ & $\begin{array}{c}0 \\
\tilde{1} \\
m\end{array}$ & $\begin{array}{l}0 \\
0 \\
1\end{array}$ & $\begin{array}{l}\tilde{1} \\
\hat{m}\end{array} \mid$ & iे & $\begin{array}{l}\infty \\
\dot{p} \\
\tilde{n}\end{array}$ & $I$ & $\stackrel{I}{I}$ & $I$ & $I$ & $I$ & $I$ \\
\hline 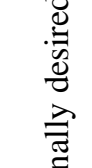 & 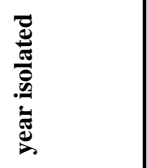 & $\begin{array}{l} \pm \\
\sigma\end{array}$ & $\stackrel{\circ}{\circ}$ & $\stackrel{\sim}{\stackrel{\sim}{\sim}}$ & $\frac{n}{\tilde{N}}$ & $\stackrel{m}{\grave{i}}$ & $\stackrel{\vec{\sim}}{\vec{\sim}}$ & $\stackrel{0}{\circ}$ & $\overline{\bar{i}}$ & $\overline{\vec{\nabla}}$ & $\overline{\vec{i}}$ & $\overline{\vec{i}}$ & $\overline{\vec{\sim}}$ \\
\hline 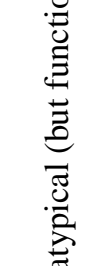 & 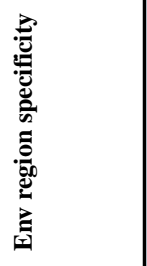 & 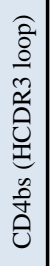 & 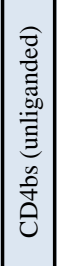 & 㝘 & Oे & 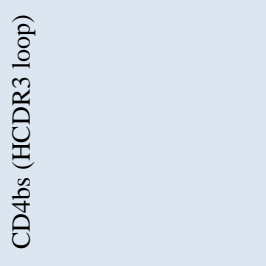 & 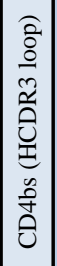 & 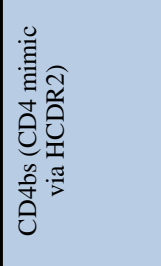 & 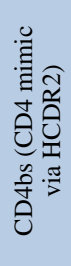 & 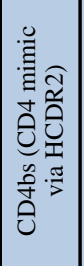 & 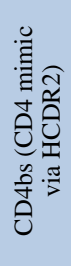 & 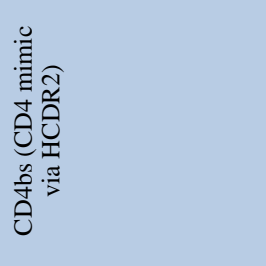 & 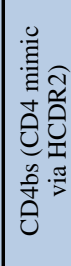 \\
\hline 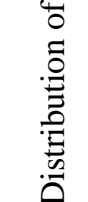 & 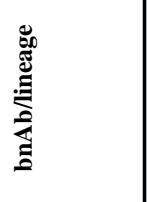 & $\frac{2}{3}$ & 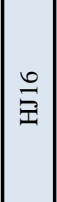 & \begin{tabular}{|l|}
$\stackrel{m}{\nu}$ \\
$\stackrel{y}{>}$
\end{tabular} & $\begin{array}{l}\stackrel{0}{0} \\
\stackrel{0}{>}\end{array}$ & $\begin{array}{l}\frac{8}{1} \\
\frac{\delta}{0} \\
\text { 전 }\end{array}$ & $\begin{array}{l}\stackrel{\infty}{0} \\
\stackrel{\Xi}{己}\end{array}$ & 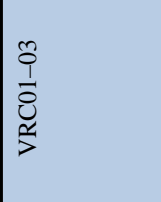 & 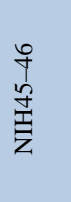 & 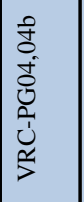 & 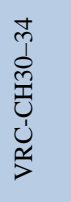 & $\begin{array}{l}8 \\
\substack{Z \\
m \\
m}\end{array}$ & 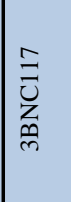 \\
\hline
\end{tabular}

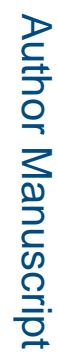

로를

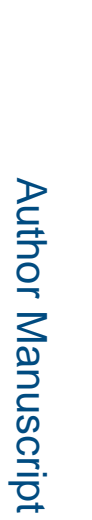




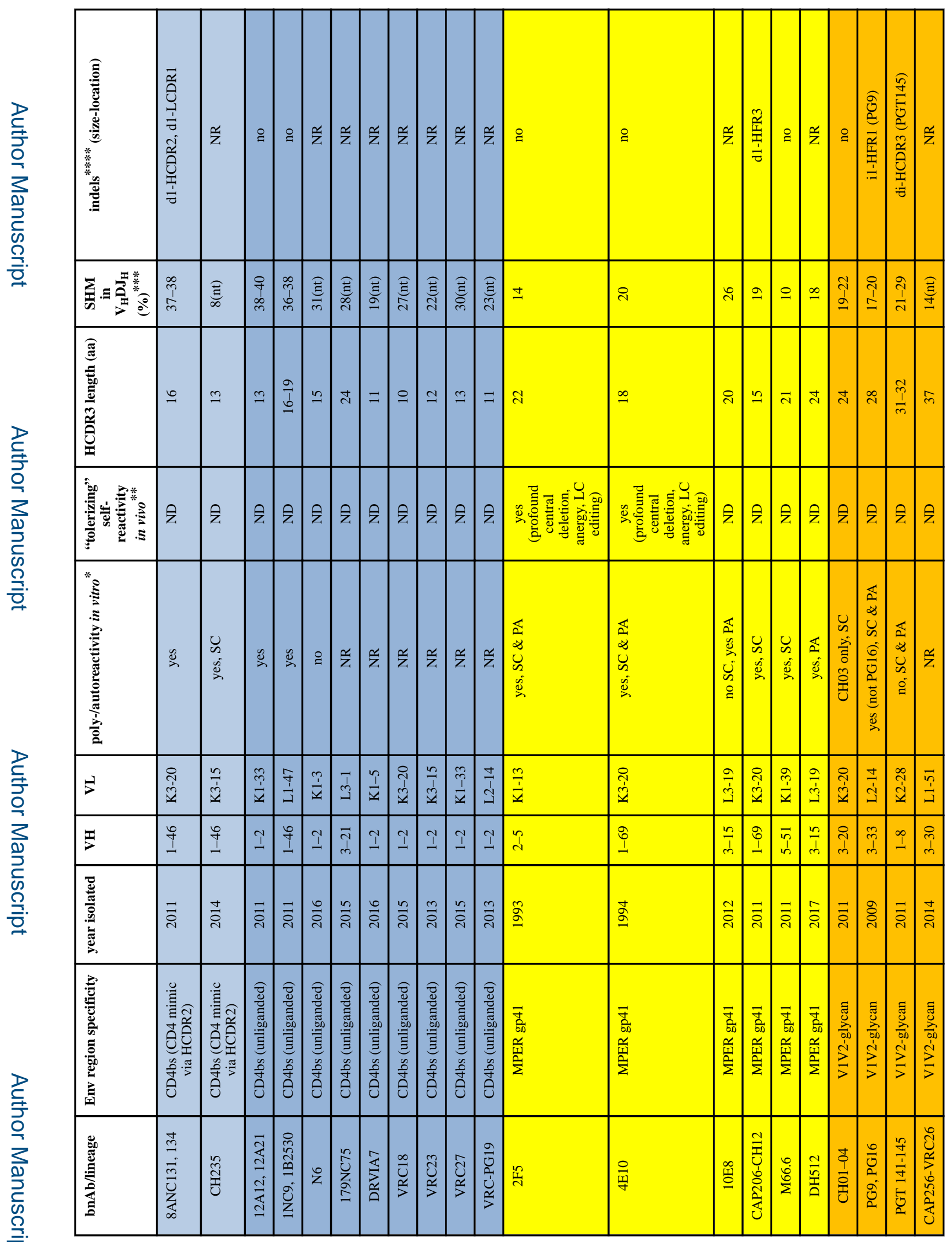




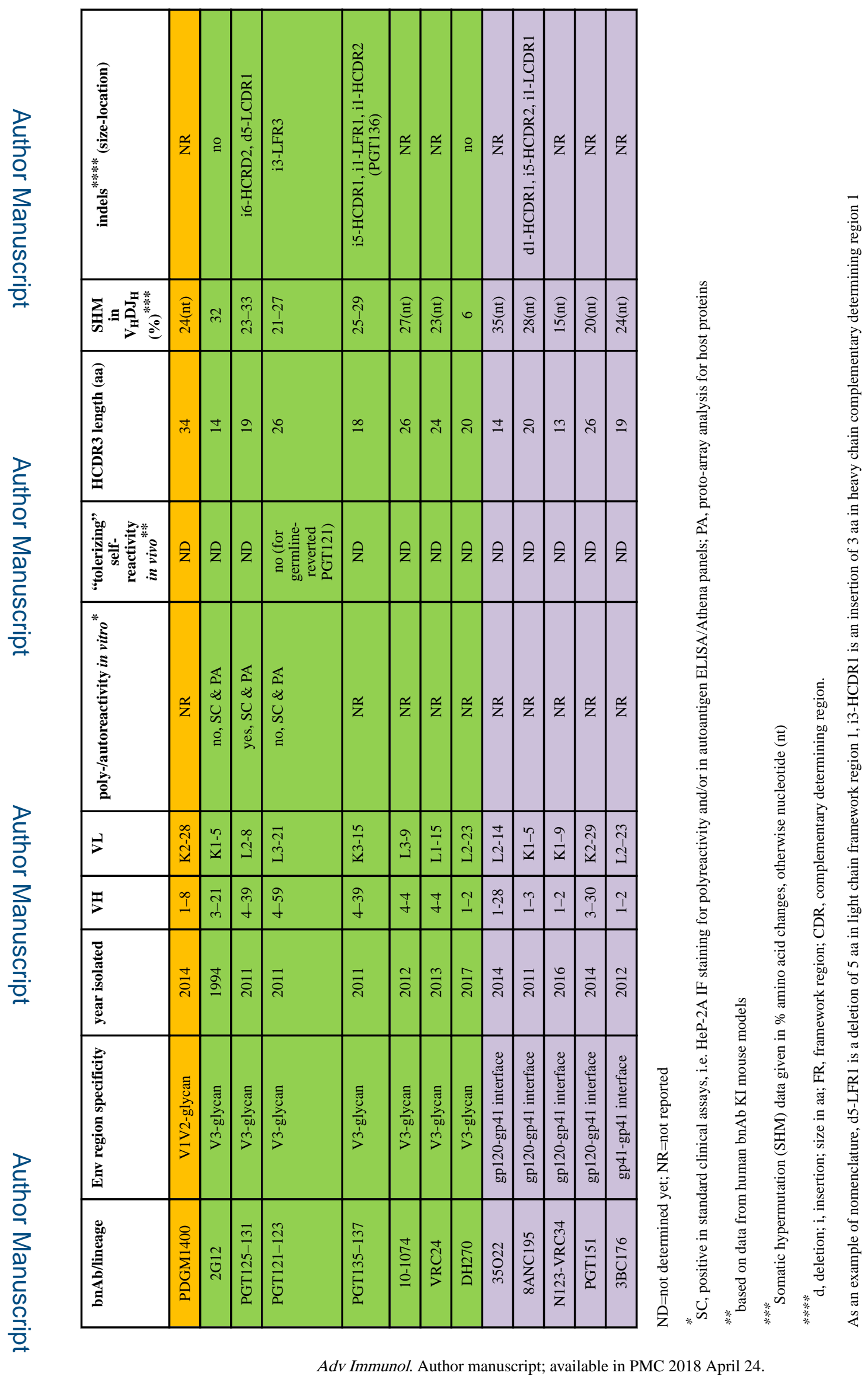




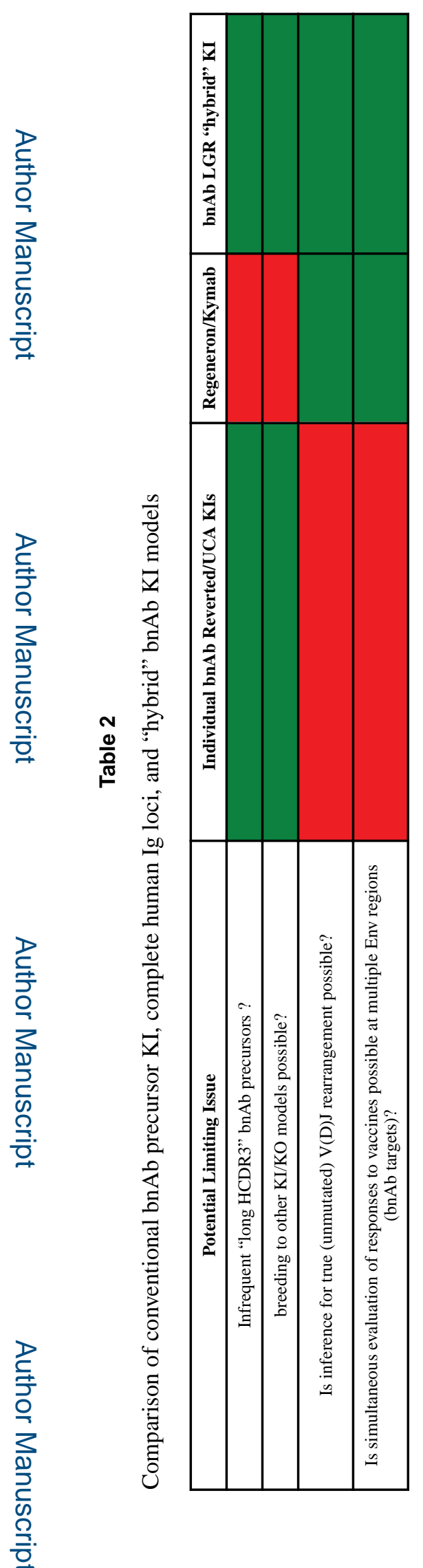

Adv Immunol. Author manuscript; available in PMC 2018 April 24. 